Supplementary Materials for:

\title{
Expanding biosensing abilities through computer-assisted design of metabolic pathways
}

Vincent Libis ${ }^{1,2}$, Baudoin Delépine ${ }^{1,2}$, Jean-Loup Faulon ${ }^{1,2,3^{*}}$

Affiliations:

1) Micalis Institute, INRA, Jouy en Josas, FRANCE

2) Institute of Systems and Synthetic Biology, CNRS, Évry, FRANCE

3) SYNBIOCHEM Center, Manchester Institute of Biotechnology, School of Chemistry, University of Manchester, Manchester, ENGLAND

Corresponding Author:

E-mail: jean-loup.faulon@jouy.inra.fr

The file include:

Fig. S1. Extended metabolic space around the DrugBank dataset.

Fig. S2. Monitoring of the response time to hippurate (a), cocaine (b) and benzoate of strains equipped with SEMP.

Fig S3. Summary of the SEMP successfully implemented in vivo over the course of this work.

Fig S4. Architecture of metabolic and sensing modules.

Fig S5. Dose response of control strains lacking metabolic module to the different compounds

Fig S6. Summary of SEMP-associated specificity modes of failure

Fig S7. Flow cytometry analysis of strains equipped with SEMP in presence of toxic concentrations of inducer

Fig S8. Growth curves of the different strains in presence of the associated compounds

Fig S9. Swing and RFP/OD of all strains with every compounds

Data file S1. Raw and calculated data

Data file S2. Sequences of plasmids used in this study 


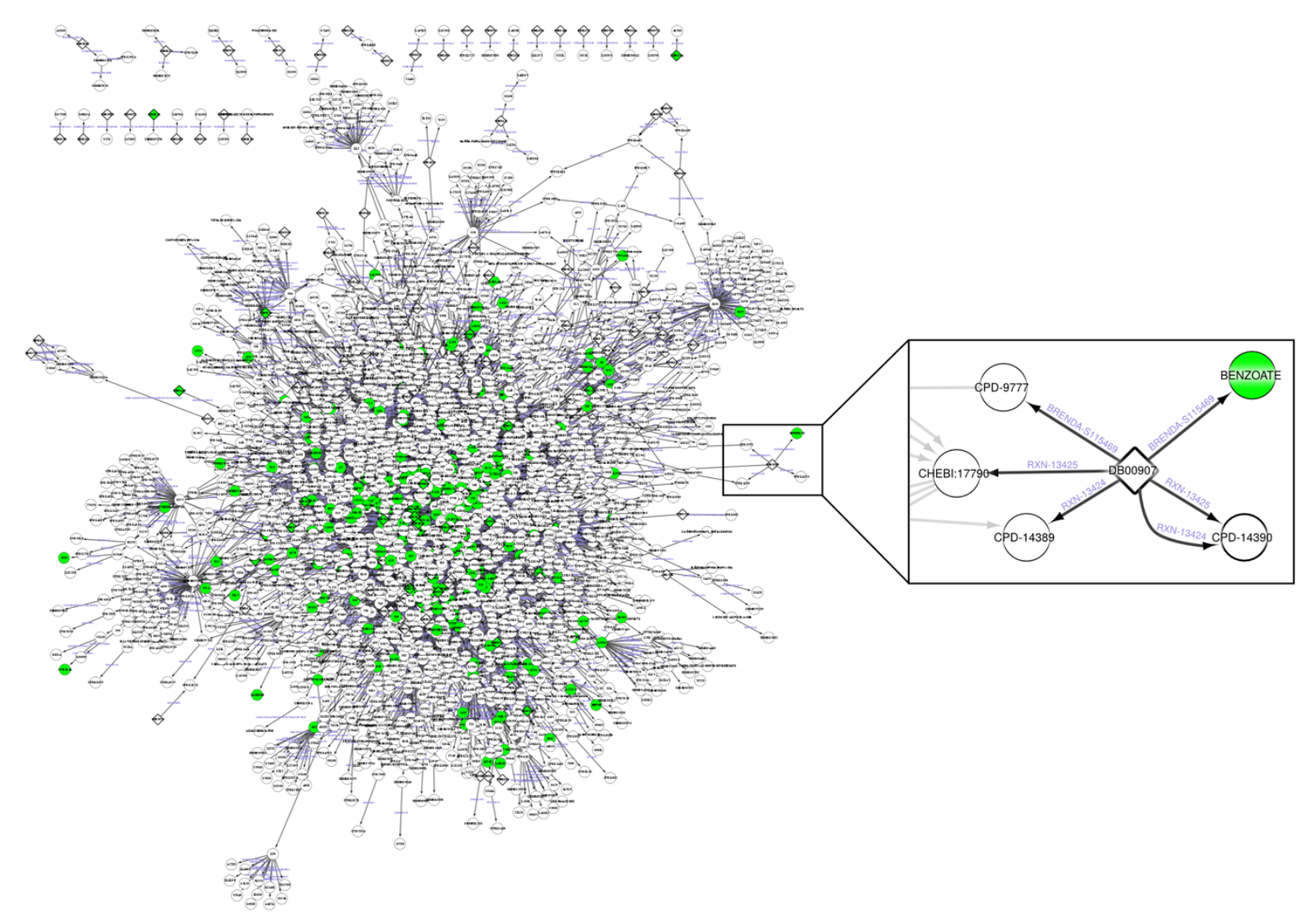

figure S1: Extended metabolic space around the DrugBank dataset. This is an oriented hypergraph generated by the bioinformatic pipeline described in Fig. 2, here visualized with Cytoscape. Similar graphs were generated and (computationally) analyzed for the HMDB and Tox21 datasets. Nodes represent chemical compounds and edges represent biochemical reactions. Each compound is associated strictly to one node. Enzymes can be associated to different pairs of compounds, and thus can be associated to several edges. Diamond shaped nodes represent target compounds from DrugBank (illicit or accepted drugs) and circular shaped nodes represent compounds that are generated through up to three metabolic steps from the initial target compounds. Nodes colored in green are naturally detectable compounds found in the transcription factor effector databases. A zoom on the area around target compound DB00907 (cocaine) shows the predicted products of different enzymatic reactions including the natural effector 
benzoate. Compounds not involved with any reaction ("non-processed compounds") are not represented. 
a)

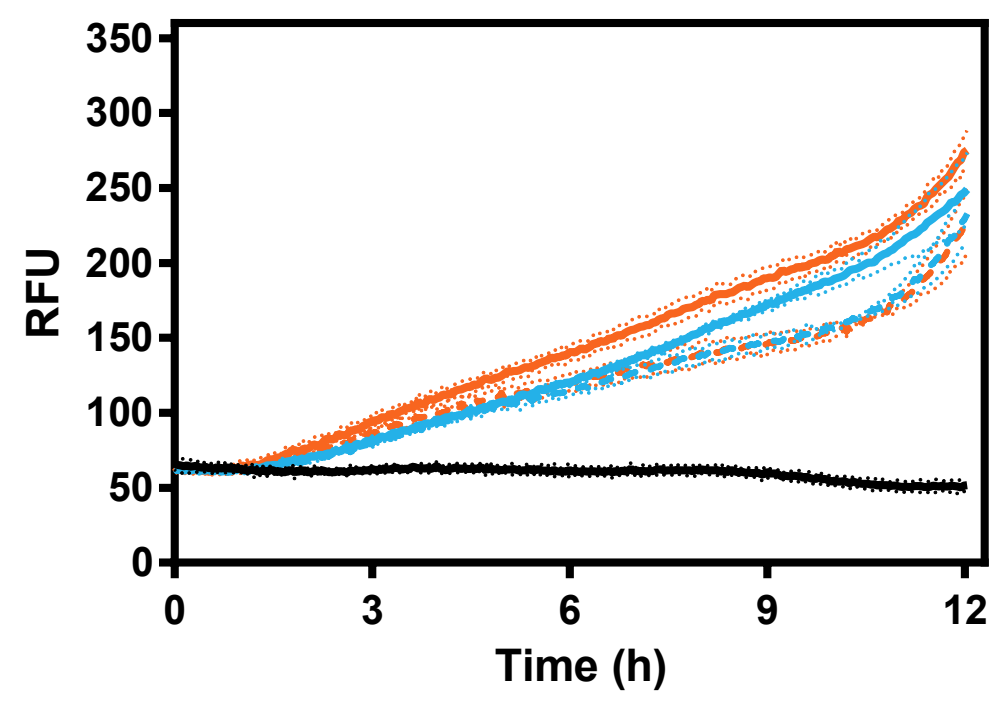

- LB control

- Hippurate $100 \mu \mathrm{M}$

- Hippurate $500 \mu \mathrm{M}$

-. Benzoate $100 \mu \mathrm{M}$

Benzoate $500 \mu \mathrm{M}$

b)

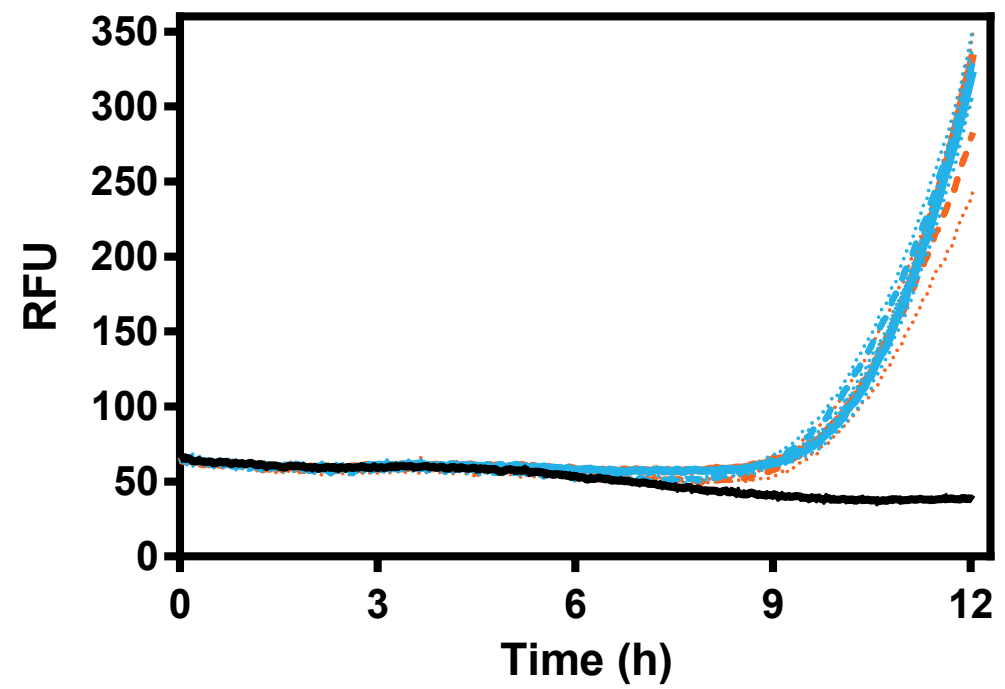

- LB control

-. Cocaine $100 \mu \mathrm{M}$

- Cocaine $500 \mu \mathrm{M}$

- - Benzoate $100 \mu \mathrm{M}$

- Benzoate $500 \mu \mathrm{M}$

Figure S2: Monitoring of the response time to hippurate (a), cocaine (b) and benzoate of strains equipped with SEMP. No delay is observed between response time to the natural inducer benzoate and to the SEMP targets hippurate and cocaine whether in presence of $100 \mu \mathrm{M}$ or $500 \mu \mathrm{M}$ of inducers. Cells were prepared in the same way as for dose-response characterization experiments (see Methods). The longer response time for the cocaine sensor might be explained by the difference of temperature at which the experiment was performed $\left(30^{\circ} \mathrm{C}\right.$ instead 
of $37^{\circ} \mathrm{C}$ for hippurate sensor). RFU stands for relative fluorescence units and consists in the raw fluorescence value measured by the platereader. Dashed lines represent standard deviation of the three replicates. 


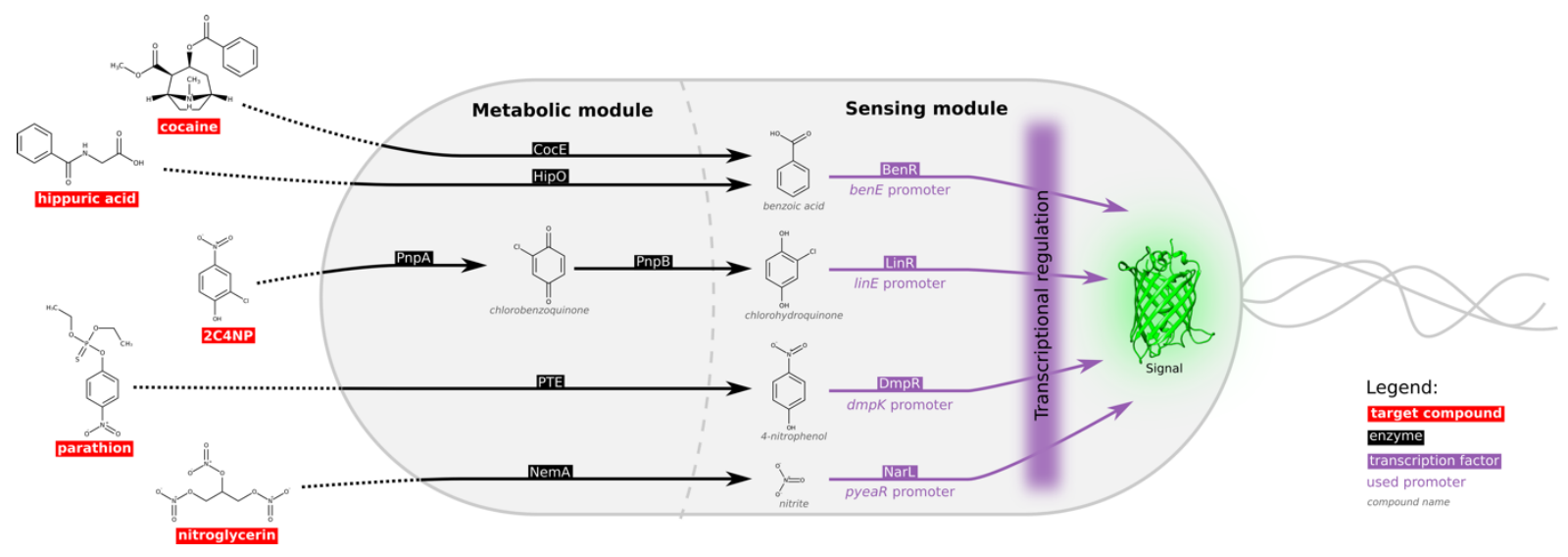

Figure S3: Summary of the SEMP successfully implemented in vivo over the course of this work. Only one pair of the represented metabolic and sensing modules was transformed in each strain. Implementation of a sixth SEMP allowing detection of the pollutant chlorpropham (see Table 1) was not successful because we were enable to obtain a response to 3-chloroaniline in E. coli based on the TadR transcription factor from Delftia tsuruhatensis. This failure may be due to a gene induction mechanism that necessitates compatibility with the host transcription machinery or that requires a co-inducing protein. Intuitively, the choice of SEMP elements coming from organisms closely related to the host in terms of evolution is expected to maximize chances of successful implementation. 
a)

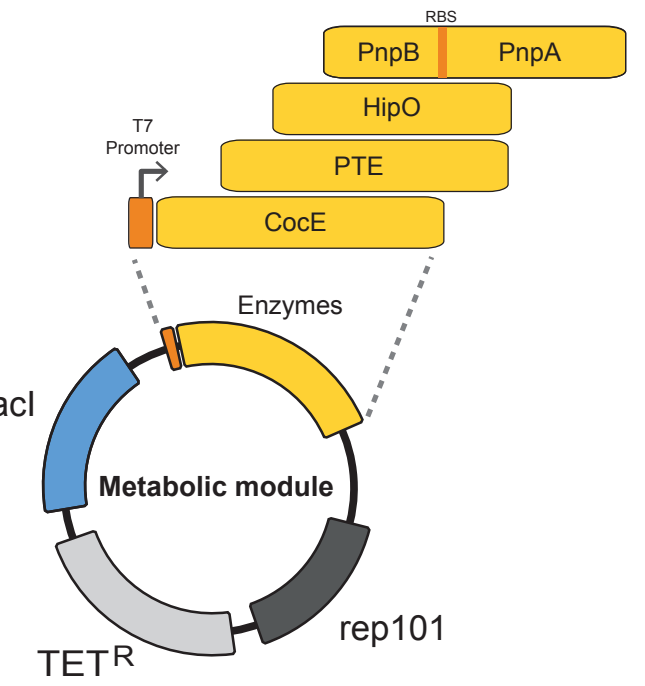

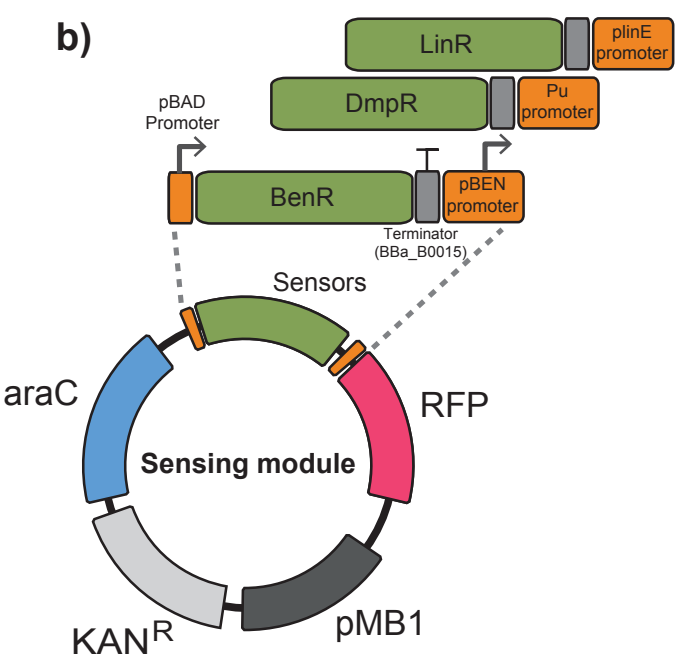

Figure S4: Architecture of metabolic and sensing modules. (a) Map of the metabolic module harboring either CocE, PTE-S5, pnpB-pnpA or HipO coding sequences. (b) Map of the sensing module harboring either BenR-pBen, DmpR-Pu or LinR-plinE sequences to control RFP. Transcriptional isolation is ensured by BBa_B0015, a double terminator from the registry of standard biological parts. 
a)

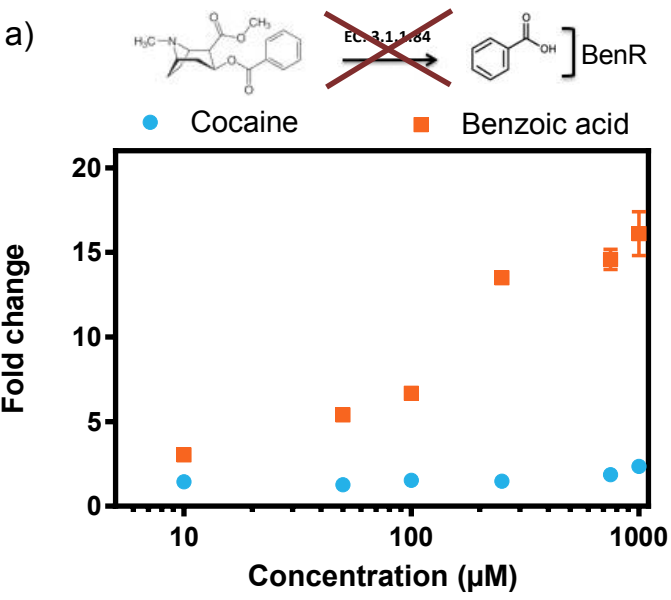

c)
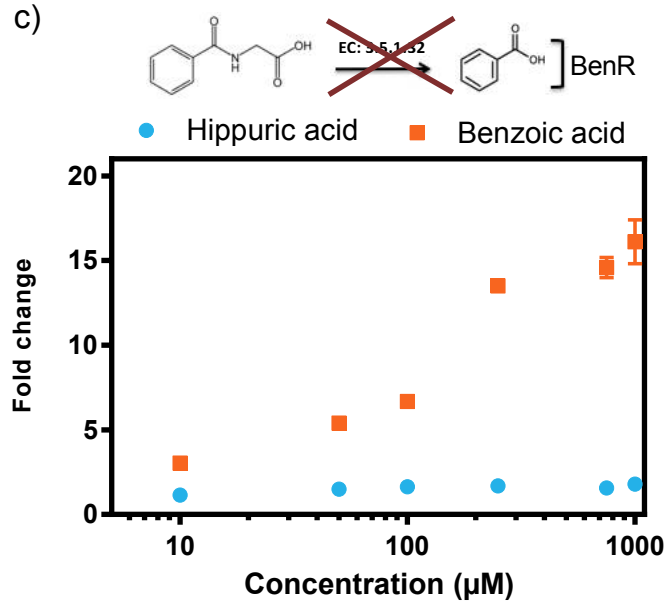

b)

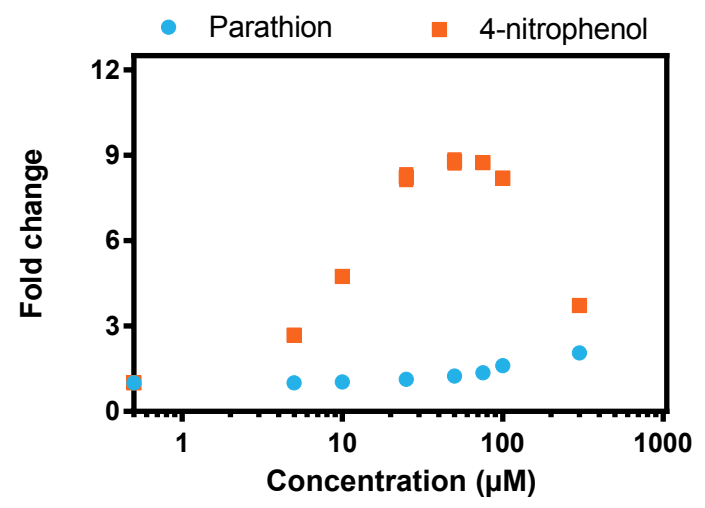

d)
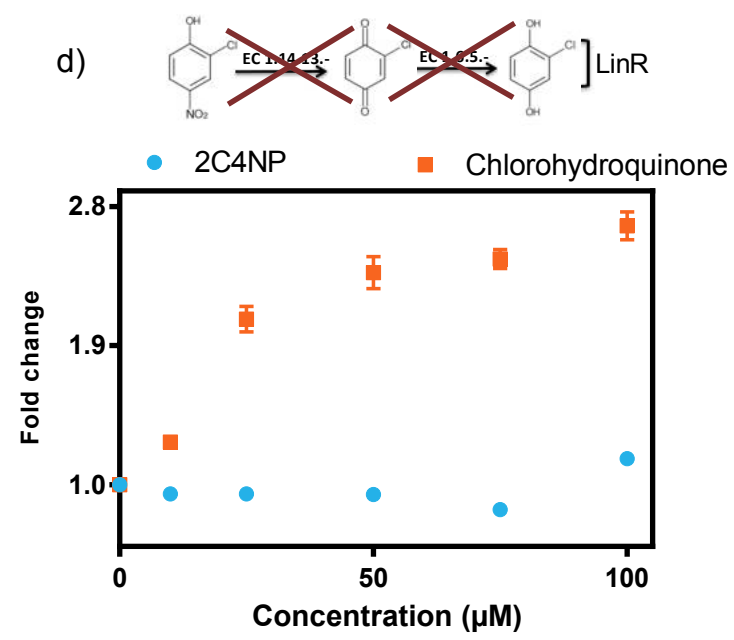

Figure S5: Dose response of control strains lacking metabolic module to the different compounds. (a)(c) Benzoate sensing module alone; (b) 4-nitrophenol sensing module alone; (d) chlorohydroquinone sensing module alone. Dosedependent response is limited to the natural effector of transcription factors BenR, DmpR, LinR (benzoic acid, 4-nitrophenol, chlorohydroquinone) when metabolic module is not present to enable sensing scope extension to target molecules. At high concentration of target molecules, traces of signal appeared even without metabolic module, this may be due to partial spontaneous hydrolysis, impurities from synthesis or promiscuity of the transcription factor. 


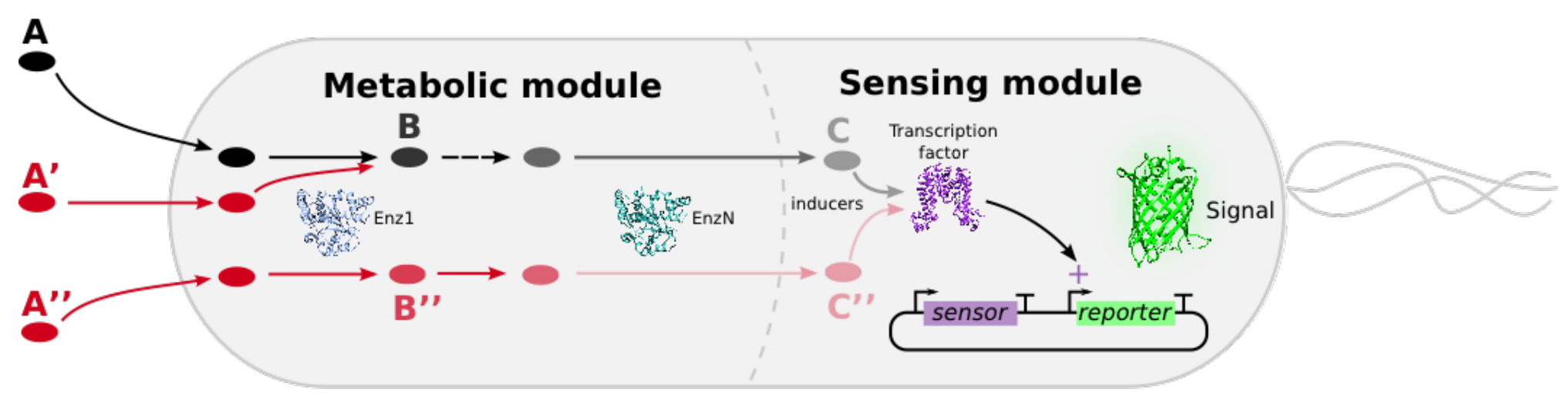

Figure S6: Summary of SEMP-associated specificity modes of failure. A specific limitation of SEMPs resides in their inability to discriminate between the presence in the medium of the target molecule (A) or of any of the intermediates of the SEMP (B, C). In the context of whole cell biosensors, this can be circumvented by the use of control strains lacking enzymatic steps to allow discrimination. Another specificity issue might emerge from the fact that both transcription factors and enzymes can display high levels of ligands promiscuity. It is interesting to note that an important degree of promiscuity of several elements composing a SEMP (enzymes, transcription factors) does not necessarily translate into poor sensor discrimination abilities. Discrimination issues only emerge either if the first step of the pathway can transform several input compounds into the same output metabolite transmitted to the downstream steps of the SEMP (A') or if structurally different output metabolites can also serve as effectors to the transcription factor ( $\mathrm{A}^{\prime \prime}$ ) (in this case both the first enzyme and the transcription factor must be promiscuous). 
a) BL21: HipO metabolic module + benzoate sensing module
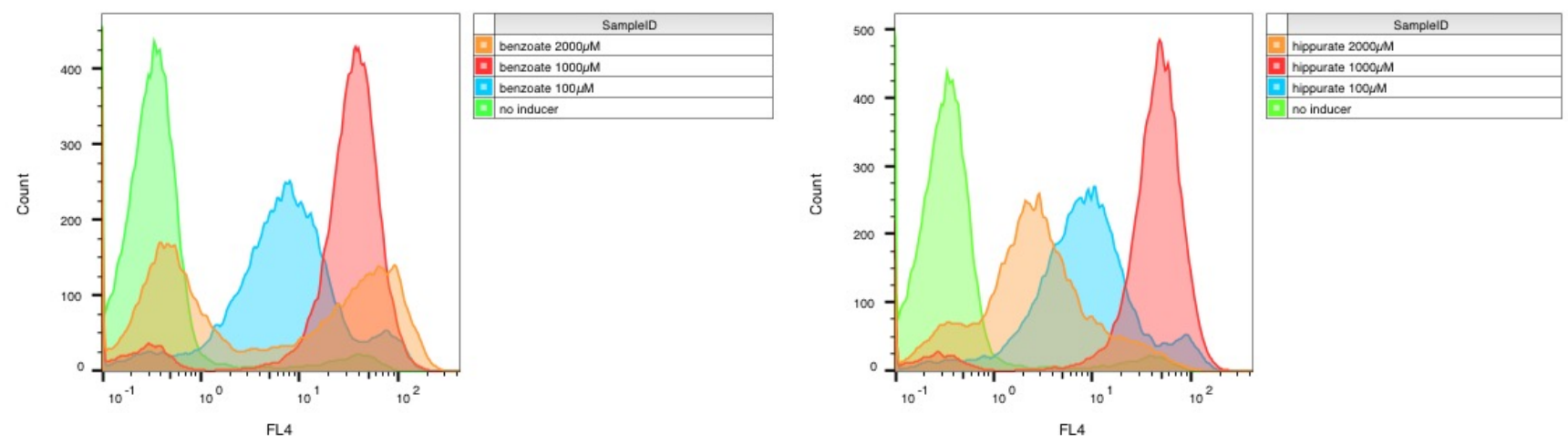

b) BL21: PTE-S5 metabolic module + 4-nitrophenol sensing module
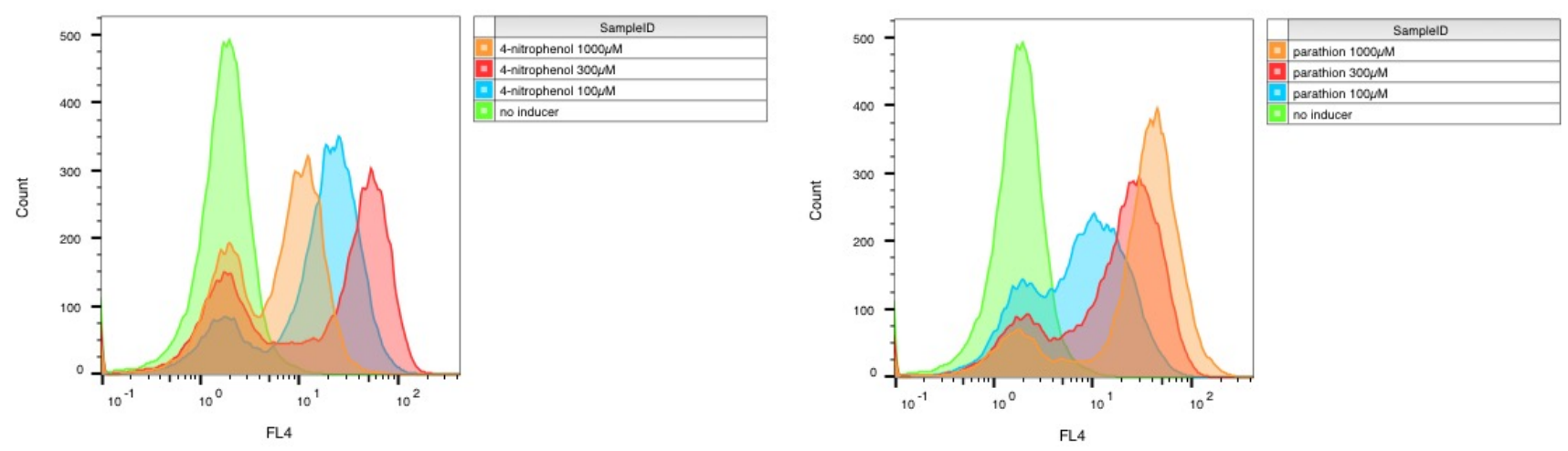

c) BL21: pnpA-pnpB metabolic module + chlorohydroquinone sensing module
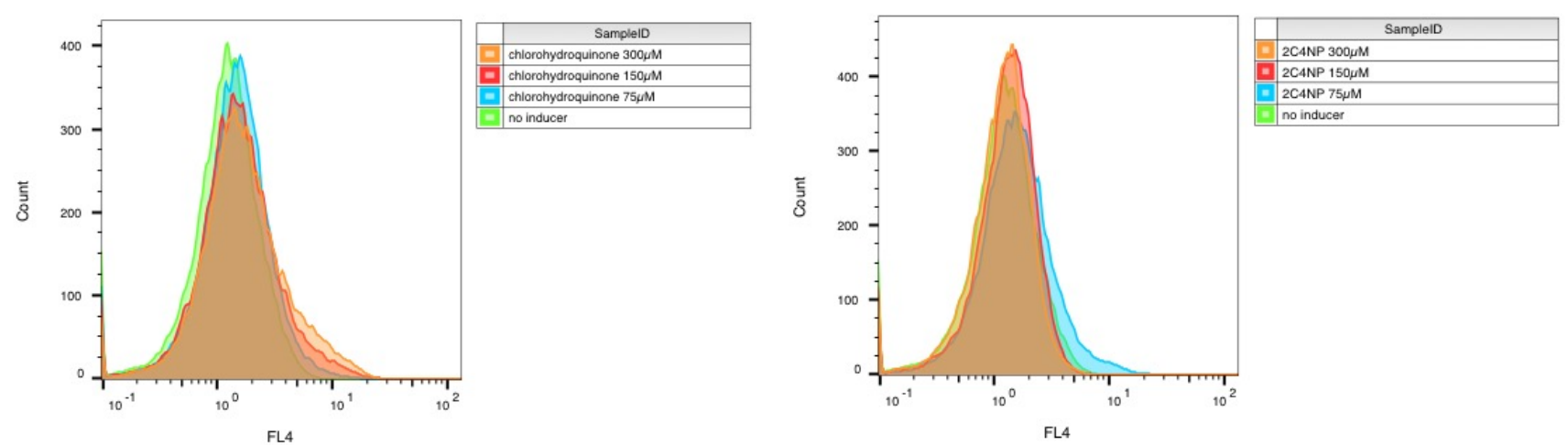

Figure S7: Flow cytometry analysis of strains equipped with SEMP in presence of toxic concentrations of inducer. Cells were cultivated in the same conditions as for dose-response characterization (see Methods) and red fluorescence was 
analysed on a CyFlow Space cytometer (PARTEC) counting 20,000 events for each sample. (a) Toxicity of benzoic acid leads to bimodal distribution of cell fluorescence at $2 \mathrm{mM}$ and half of the population emits more fluorescence than with lower concentrations. Interestingly, hippuric acid toxicity has a different effect on cell response as fluorescence at $2 \mathrm{mM}$ decreases below the level observed with $100 \mu \mathrm{M}$.

(b) Toxicity of 4-nitrophenol leads to lower level of fluorescence at $2 \mathrm{mM}$ than at $100 \mu \mathrm{M}$, similarly to what is observed with hippuric acid. On the contrary, proportionality of the response to parathion is maintained even at toxic levels. As shown in suppl. Fig 8, parathion by itself is not toxic unless PTE-S5 enzyme is present to transform it into 4-nitrophenol. This is probably the cause of the robust graded fluorescent response at high concentration although the exact mechanism is unknown. (c) Despite the low dynamic range of LinR, a small but graded response to chlorohydroquinone is observed while response to 2C4NP only occurs at low concentration $(75 \mu \mathrm{M})$ and is completely abolished upon reaching toxic levels. 
a) BL21: PTE-S5 metabolic module + 4-nitrophenol sensing module
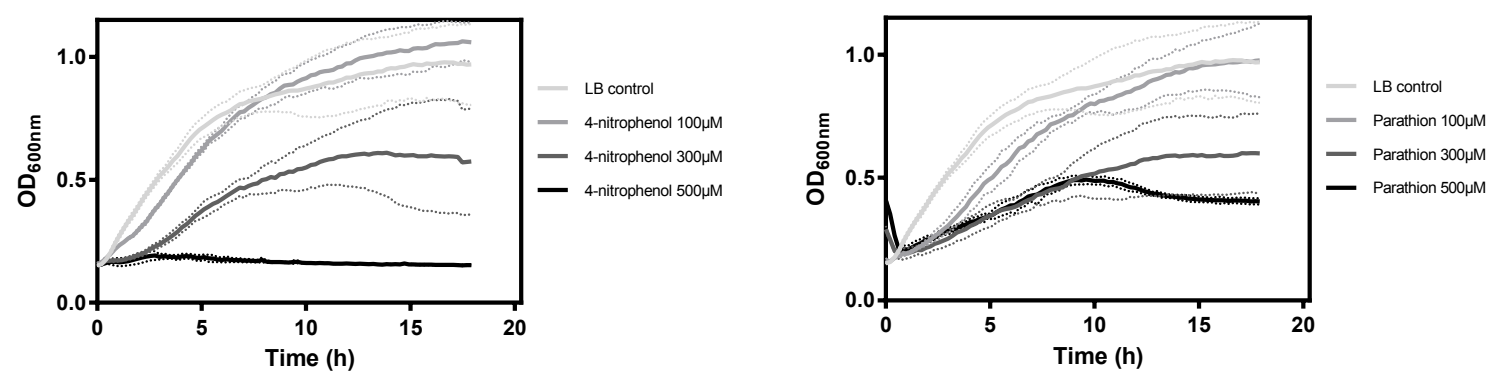

b)

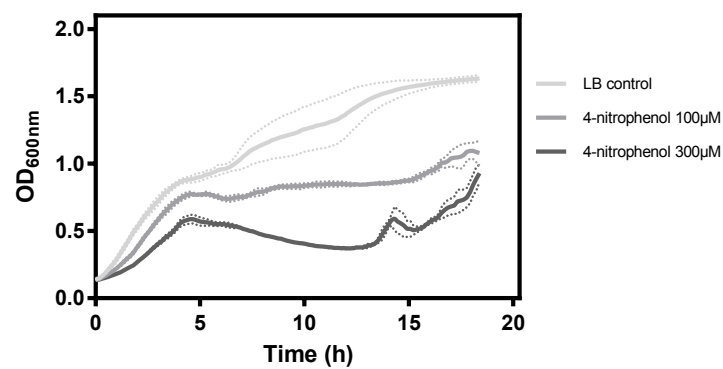

BL21: 4-nitrophenol sensing module

C) BL21: pnpB-pnpA metabolic module + chlorohydroquinone sensing module
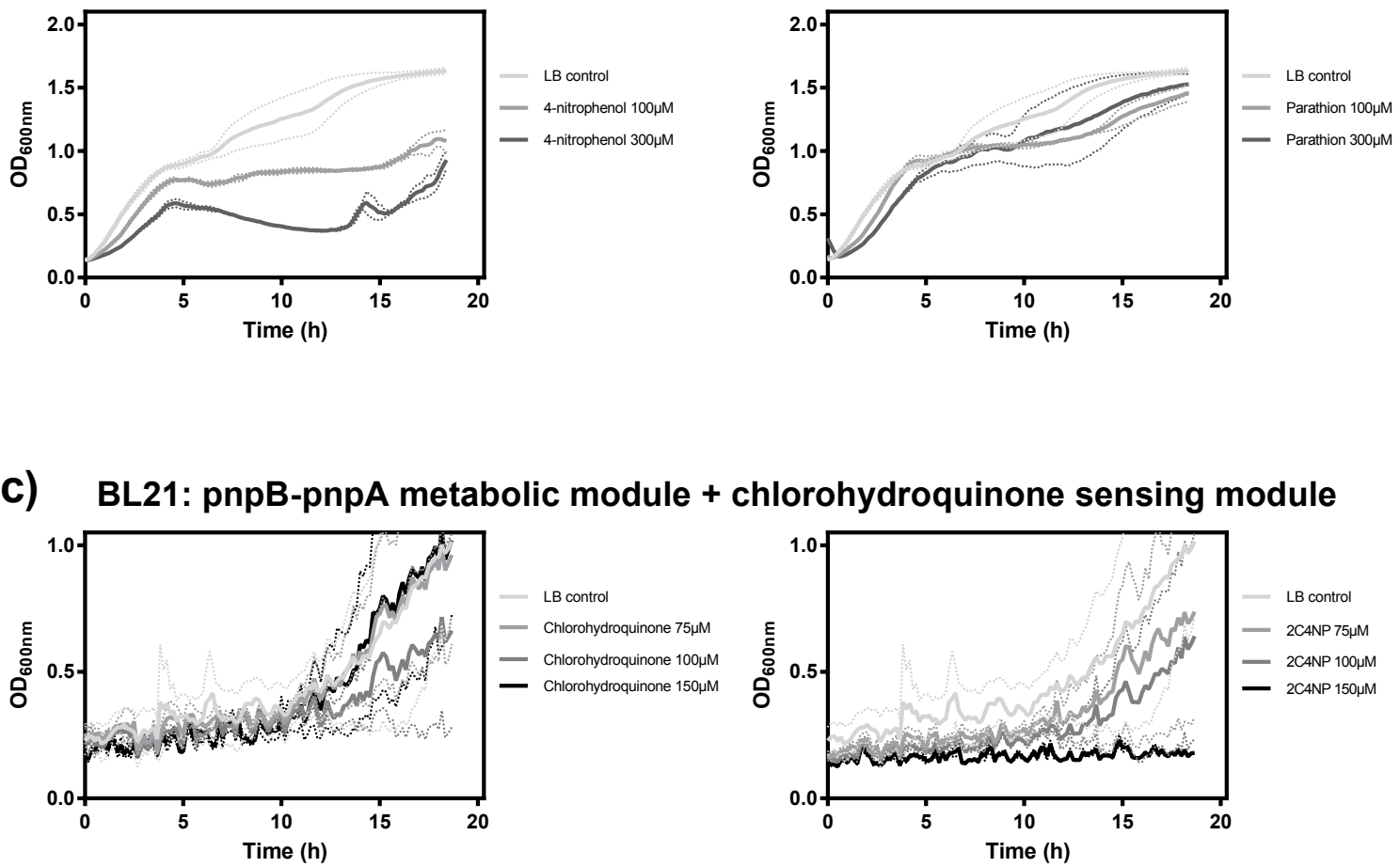

d)
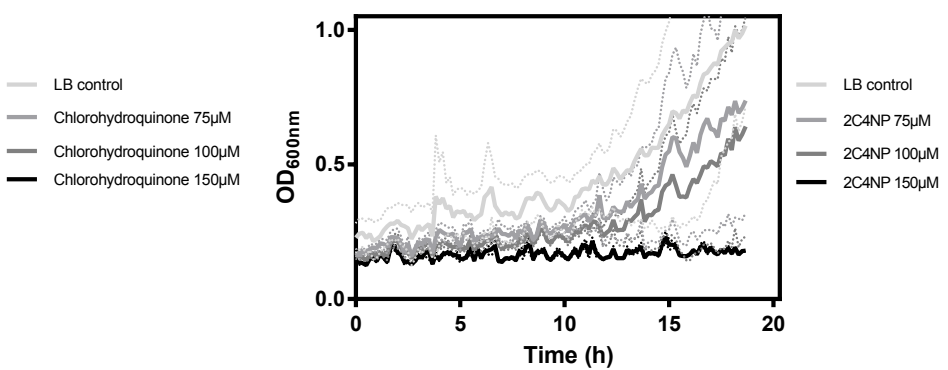

BL21: chlorohydroquinone sensing module
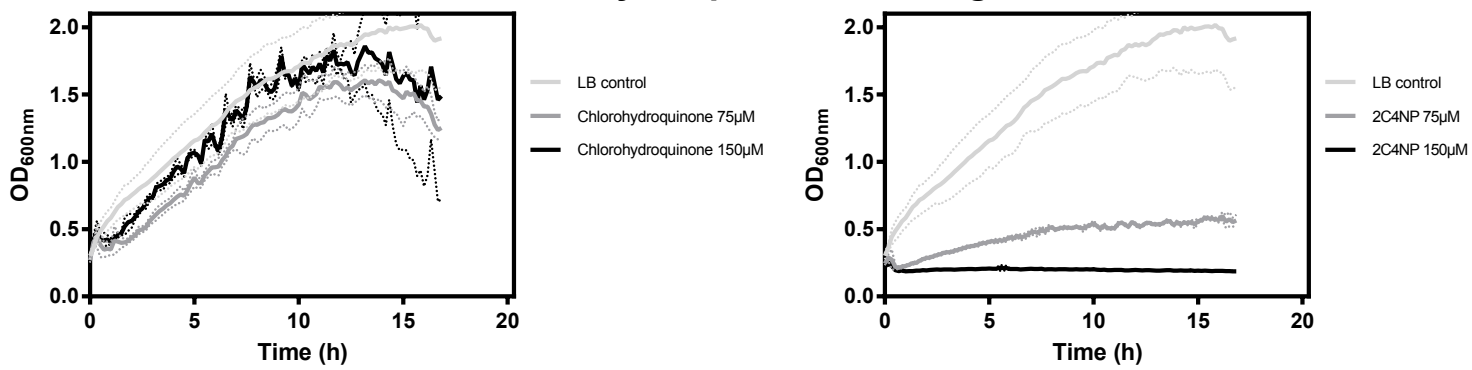
e) BL21: CocE metabolic module + benzoate sensing module
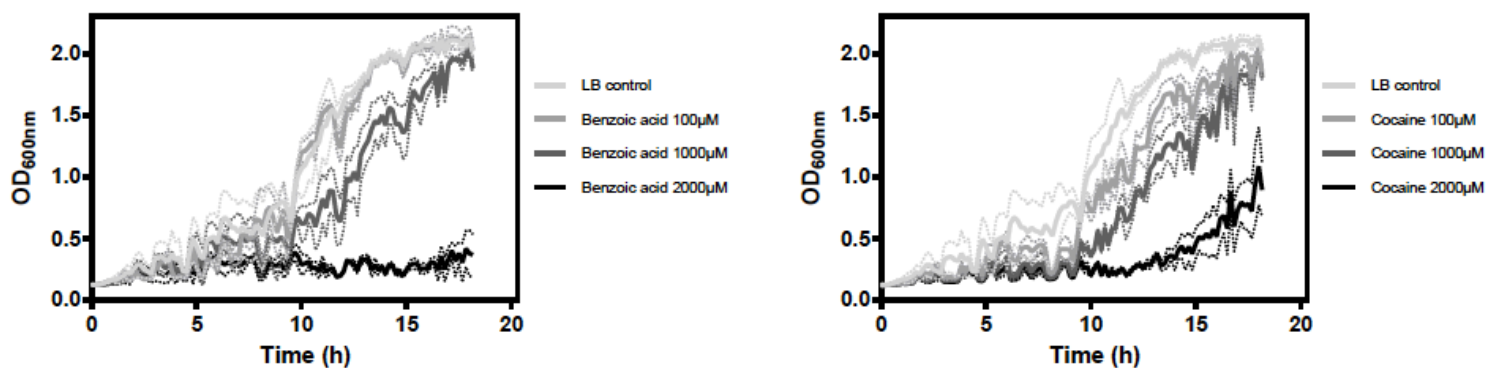

f) BL21: HipO metabolic module + benzoate sensing module
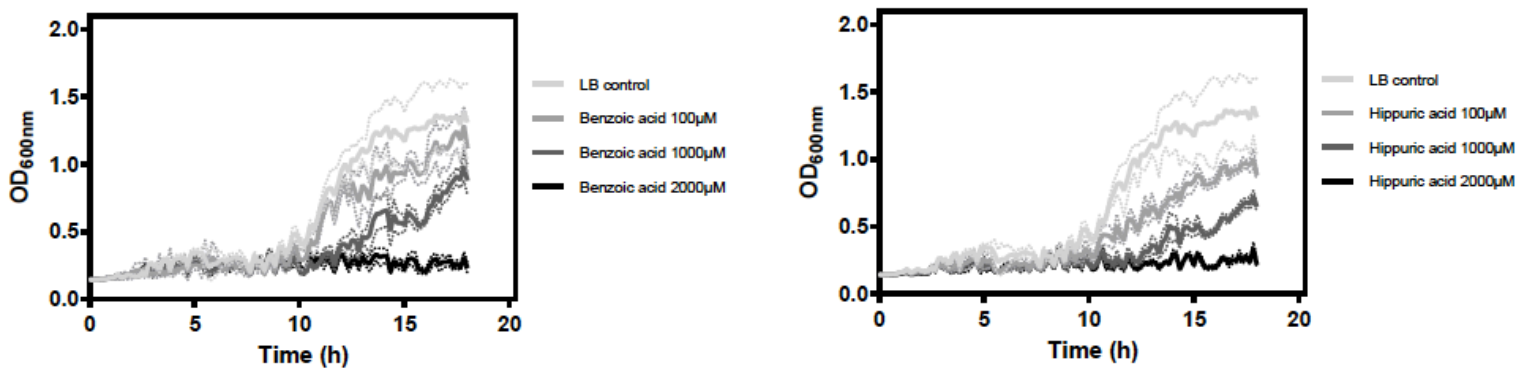

g)

BL21: benzoate sensing module
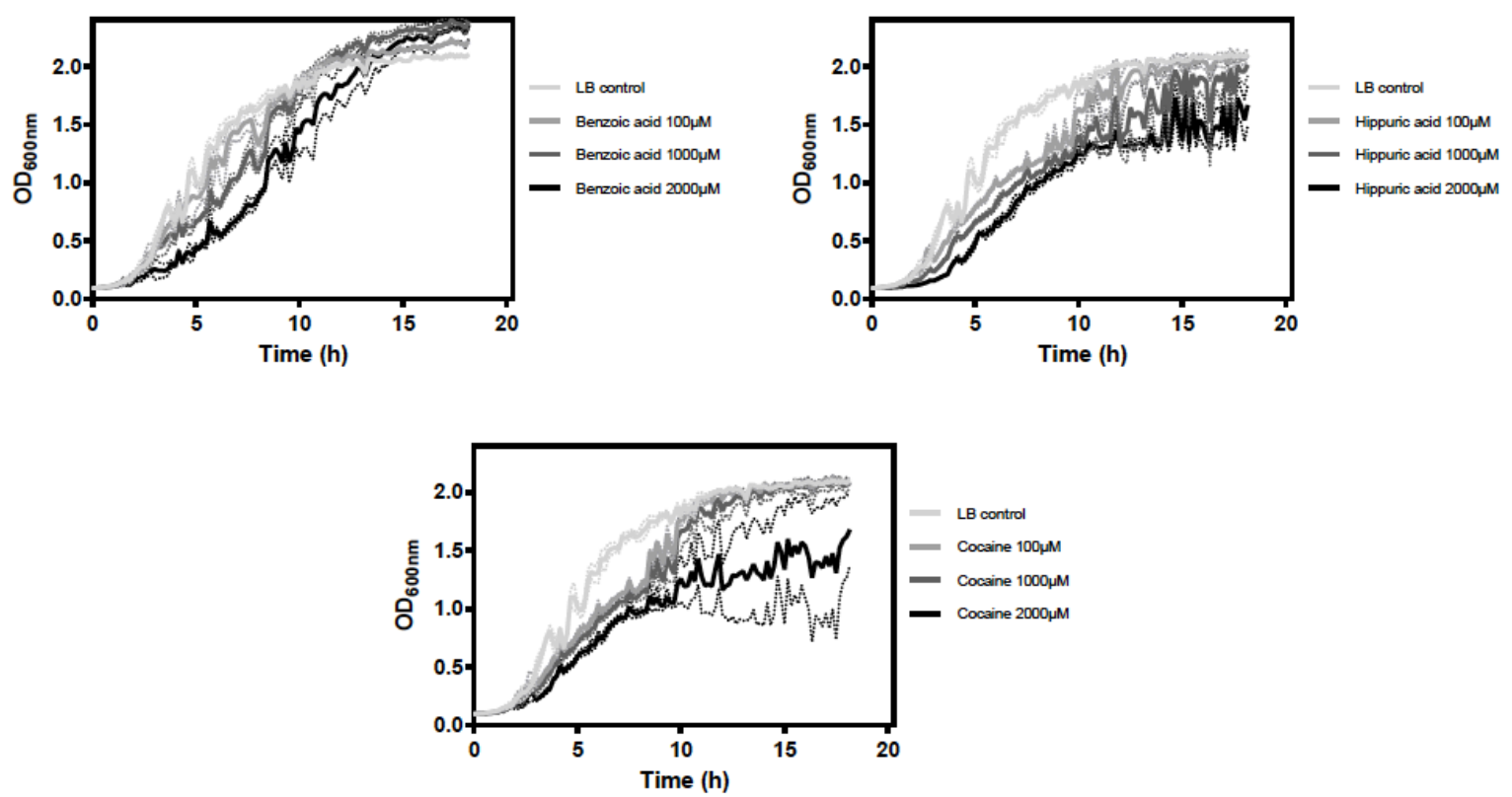

Fig S8. Growth curves of the different strains in presence of the associated compounds. 
BL21: CocE metabolic module + benzoate sensing module
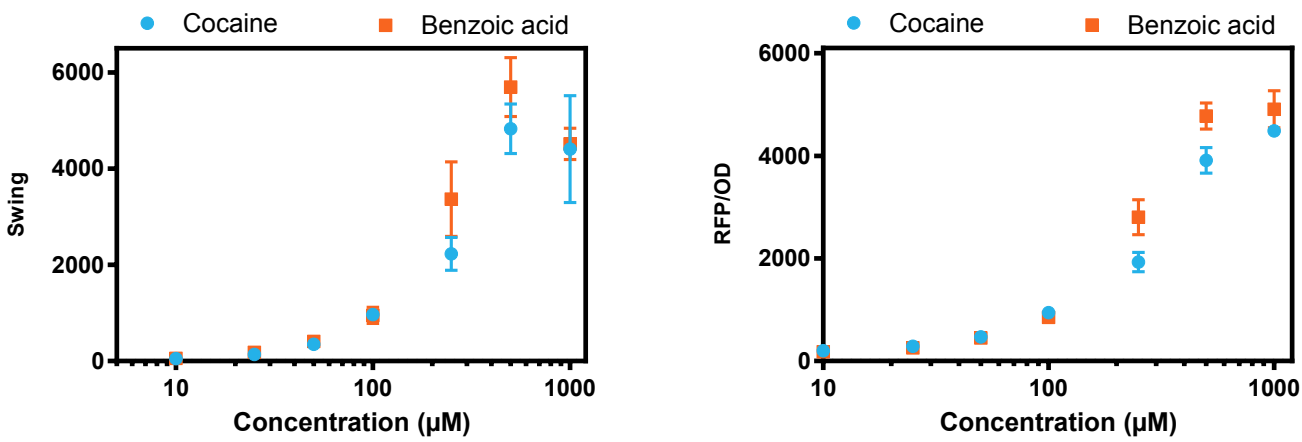

BL21: PTE-S5 metabolic module + 4-nitrophenol sensing module
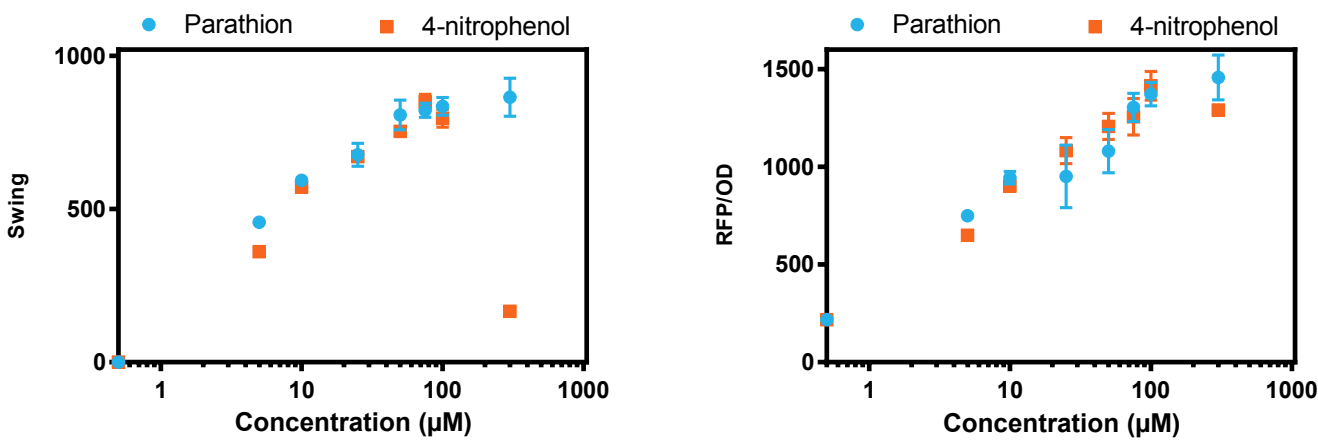

BL21: HipO metabolic module + benzoate sensing module
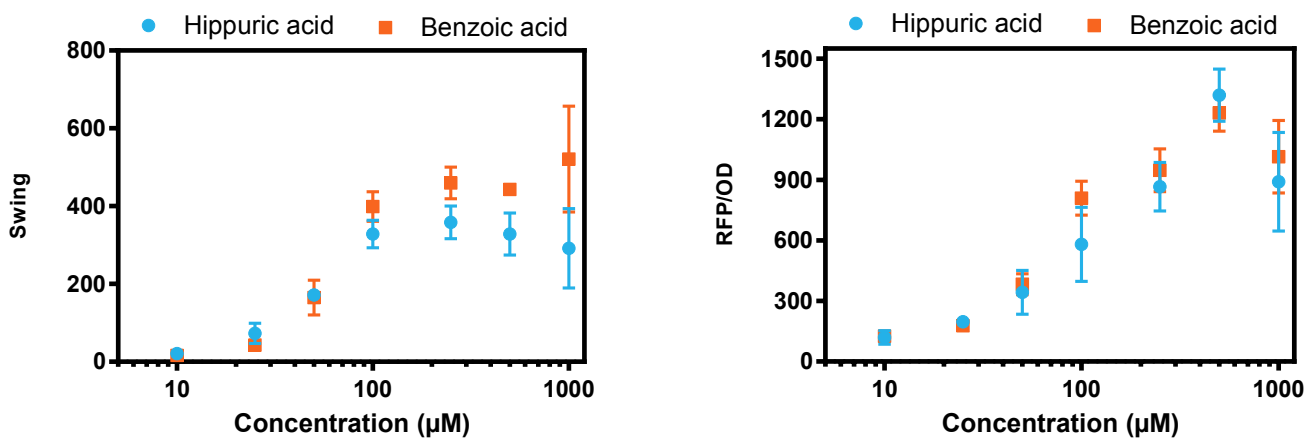

BL21: pnpB-pnpA metabolic module + chlorohydroquinone sensing module
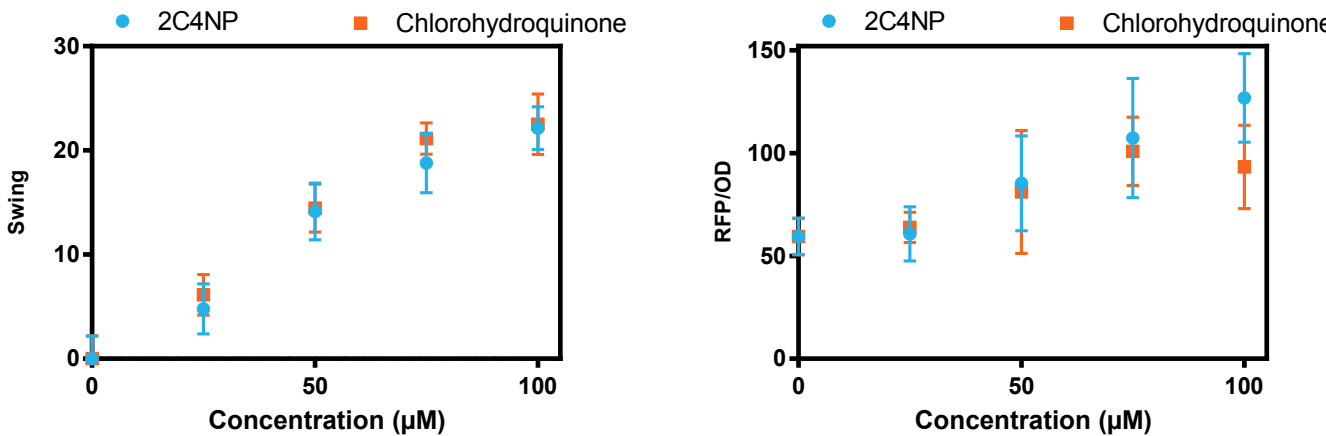
BL21: benzoate sensing module
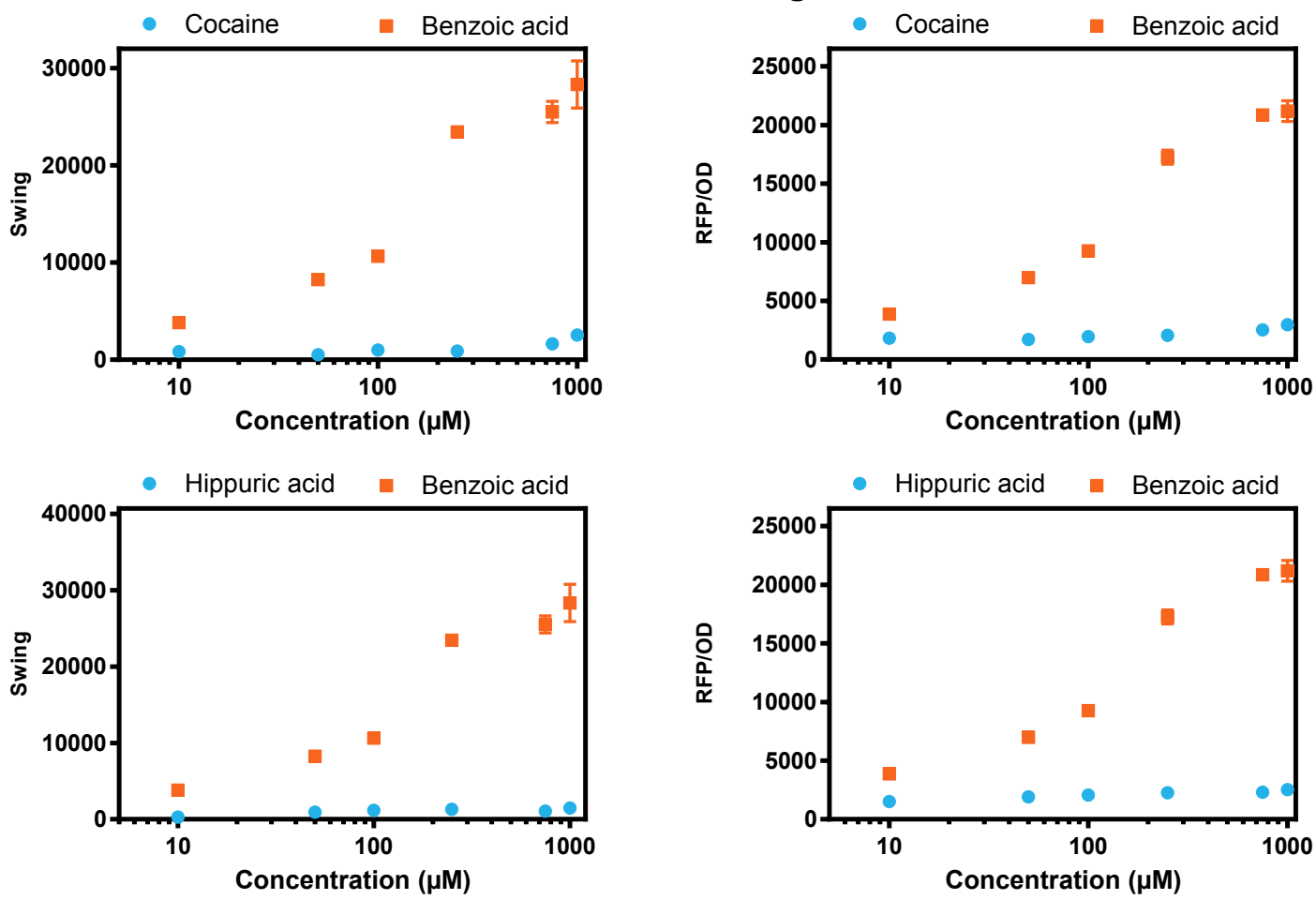

\section{BL21: 4-nitrophenol sensing module}
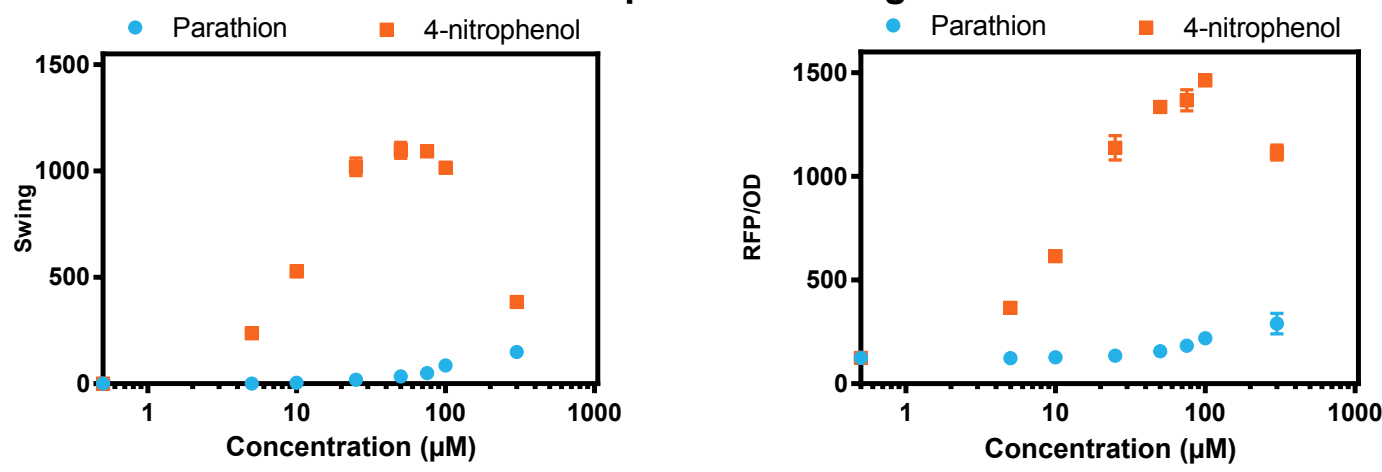

\section{BL21: chlorohydroquinone sensing module}
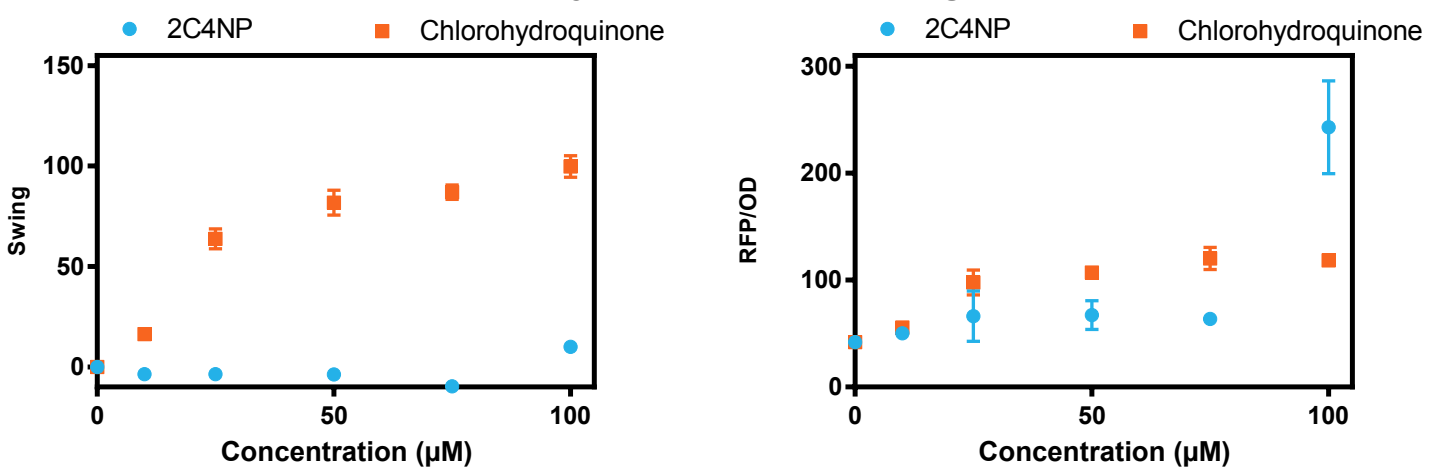
Fig S9. Swing and RFP/OD of all strains with every compounds. Swing is defined as the difference in raw fluorescence observed between induced cells and noninduced control population. 
Data file S1. Raw data and calculations

BL21: CocE metabolic module + benzoate sensing module

\begin{tabular}{|c|c|c|}
\hline & 600 & RFP (a.u.) \\
\hline \multirow{3}{*}{ no inducer } & 1,295 & 166 \\
\hline & 1,1914 & 154 \\
\hline & 1,1329 & 158 \\
\hline \multirow{3}{*}{ cocaine $10 \mu \mathrm{M}$} & 1,1428 & \\
\hline & 1,0104 & 205 \\
\hline & 0,9874 & 200 \\
\hline \multirow{2}{*}{ benzoate $10 \mu \mathrm{M}$} & 1,1706 & 209 \\
\hline & 1,2377 & 226 \\
\hline \multirow{3}{*}{ cocaine $25 \mu \mathrm{M}$} & 1,1193 & 294 \\
\hline & 0,9724 & 274 \\
\hline & 1,0059 & 318 \\
\hline \multirow{3}{*}{ benzoate $25 \mu \mathrm{M}$} & 1,3077 & 313 \\
\hline & 1,3367 & 338 \\
\hline & 1,3271 & 374 \\
\hline \multirow{3}{*}{ cocaine $50 \mu \mathrm{M}$} & 1,1283 & 504 \\
\hline & 0,9065 & 455 \\
\hline & 1,2152 & 568 \\
\hline \multirow{3}{*}{ benzoate $50 \mu \mathrm{M}$} & 1,2206 & 516 \\
\hline & 1,2399 & 552 \\
\hline & 1,3005 & 631 \\
\hline \multirow{3}{*}{ cocaine $100 \mu \mathrm{M}$} & 1,1999 & 1139 \\
\hline & 1,1746 & 1080 \\
\hline & 1,2129 & 1164 \\
\hline \multirow{3}{*}{ benzoate $100 \mu \mathrm{M}$} & 1,2406 & 933 \\
\hline & 1,3357 & 1273 \\
\hline & 1,3239 & 1126 \\
\hline \multirow{3}{*}{ cocaine $250 \mu \mathrm{M}$} & 1,2087 & 2238 \\
\hline & 1,1973 & 2145 \\
\hline & 1,2941 & 2777 \\
\hline \multirow{3}{*}{ benzoate $250 \mu \mathrm{M}$} & 1,1854 & 3172 \\
\hline & 1,174 & 2992 \\
\hline & 1,3831 & 4412 \\
\hline \multirow{3}{*}{ cocaine $500 \mu \mathrm{M}$} & 1,276 & 4909 \\
\hline & 1,2202 & 4517 \\
\hline & 1,3231 & 5536 \\
\hline \multirow{3}{*}{ benzoate $500 \mu \mathrm{M}$} & 1,2313 & 5969 \\
\hline & 1,1554 & 5194 \\
\hline & 1,2837 & 6406 \\
\hline \multirow{3}{*}{ cocaine $1000 \mu \mathrm{M}$} & 1,2592 & 5792 \\
\hline & 0,802 & 3610 \\
\hline & 0,9864 & 4303 \\
\hline \multirow{3}{*}{ benzoate $1000 \mu \mathrm{M}$} & 1,077 & 4996 \\
\hline & 0,8787 & 4675 \\
\hline & 0,912 & 4350 \\
\hline
\end{tabular}

\begin{tabular}{|c|c|c|c|c|c|c|c|c|c|}
\hline & & \begin{tabular}{|l|} 
no inducer \\
\end{tabular} & $10 \mu \mathrm{M}$ & $25 \mu \mathrm{M}$ & $50 \mu \mathrm{M}$ & $100 \mu \mathrm{M}$ & $250 \mu \mathrm{M}$ & $500 \mu \mathrm{M}$ & $1000 \mu \mathrm{M}$ \\
\hline \multirow[t]{2}{*}{ means } & cocaine & 0 & 52 & 136 & 349,666667 & 968,333333 & 2227,33333 & 4828 & 4409 \\
\hline & benzoate & 0 & 54,3333333 & 182,333333 & 407 & 951,333333 & $\quad 3366$ & 5697 & 4514,33333 \\
\hline \multirow[t]{4}{*}{ std } & cocaine & 6,11010093 & 15,5026879 & 22,0302822 & 56,6656863 & 43,1315816 & 341,221824 & 513,996433 & 1114,93602 \\
\hline & benzoate & 6,11010093 & 10,7857931 & 30,664855 & 58,824598 & 170,517839 & 773,132158 & 613,804801 & 323,002064 \\
\hline & RFP/OD & & & & & & & & \\
\hline & & \begin{tabular}{|l|} 
no inducer \\
\end{tabular} & $10 \mu \mathrm{M}$ & $25 \mu \mathrm{M}$ & $50 \mu \mathrm{M}$ & $100 \mu \mathrm{M}$ & $250 \mu \mathrm{M}$ & $500 \mu \mathrm{M}$ & $1000 \mu \mathrm{M}$ \\
\hline \multirow[t]{2}{*}{ means } & cocaine & 132,303371 & 201,942374 & 286,858672 & 472,010995 & 942,797039 & 1929,66664 & 3911,04797 & 4487,77347 \\
\hline & benzoate & 132,303371 & 182,479548 & 258,010185 & 451,046036 & 851,877063 & 2804,79254 & 4777,7991 & 4909,63599 \\
\hline \multirow[t]{4}{*}{ std } & cocaine & 6,22545005 & 1,35924231 & 27,0951013 & 27,9059852 & 20,8717761 & 189,648888 & 247,393131 & 119,281188 \\
\hline & benzoate & 6,22545005 & 3,82034551 & 21,6961066 & 31,6356793 & 3100,50833 & 339,566133 & 254,727211 & 361,670825 \\
\hline & Change (f) & & & & & & & & \\
\hline & & \begin{tabular}{|l|} 
no inducer \\
\end{tabular} & $10 \mu \mathrm{M}$ & $25 \mu \mathrm{M}$ & 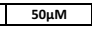 & \begin{tabular}{|l|l|}
$100 \mu \mathrm{M}$ \\
\end{tabular} & $250 \mu \mathrm{M}$ & $500 \mu \mathrm{M}$ & $1000 \mu \mathrm{M}$ \\
\hline \multirow[t]{2}{*}{ means } & cocaine & & 1,32635983 & 1,85355649 & 3,19456067 & 7,07740586 & 14,9790795 & 31,3012552 & 28,6715481 \\
\hline & benzoate & & 1,34100418 & 2,14435146 & 53,55439331 & 6,9707113 & 22,125523 & 36,7552301 & 29,332636 \\
\hline \multirow{2}{*}{ std } & cocaine & 0,03834791 & 0,0972972 & 0,13826537 & 0,35564238 & 0,2707003 & 2,14155957 & 3,22591904 & 6,99750641 \\
\hline & benzoate & & & 0,19245725 & 50,36919204 & 1,07019564 & 4,85229388 & 3,85233138 & 2,02720961 \\
\hline
\end{tabular}


BL21: benzoate sensing module

\begin{tabular}{|c|c|c|}
\hline & $600 \quad \mathrm{P}$ & $\operatorname{RFP}($ a.u.) \\
\hline \multirow{6}{*}{ no inducer } & 1,5831 & 3133 \\
\hline & 1,3088 & 1808 \\
\hline & 1,2266 & 1585 \\
\hline & 1,1706 & 1659 \\
\hline & 1,2046 & 1579 \\
\hline & 1,1924 & 1489 \\
\hline \multirow{3}{*}{ benzoate 10uM } & 1,4141 & 5320 \\
\hline & 1,5218 & 6114 \\
\hline & 1,4577 & 5652 \\
\hline \multirow{3}{*}{ cocaine $10 \mu \mathrm{M}$} & 1,4333 & 2298 \\
\hline & 1,4923 & 2680 \\
\hline & 1,5393 & 3163 \\
\hline \multirow{3}{*}{ 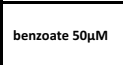 } & 1,4116 & 9534 \\
\hline & 1,477 & 10499 \\
\hline & 1,4595 & 10369 \\
\hline \multirow{3}{*}{ cocaine $50 \mu \mathrm{M}$} & 1,4003 & 2353 \\
\hline & 1,3808 & 2373 \\
\hline & 1,372 & 2422 \\
\hline \multirow{3}{*}{ benzoate $100 \mu \mathrm{M}$} & 1,4037 & 13166 \\
\hline & 1,3494 & 12028 \\
\hline & 1,3012 & 12359 \\
\hline \multirow{3}{*}{ cocaine $100 \mu \mathrm{M}$} & 1,3833 & 2423 \\
\hline & 1,5234 & 3195 \\
\hline & 1,5163 & 3028 \\
\hline \multirow{3}{*}{ benzoate $250 \mu \mathrm{M}$} & 1,4125 & 25298 \\
\hline & 1,5113 & 25964 \\
\hline & $\begin{array}{l}1,4803 \\
\end{array}$ & 24736 \\
\hline \multirow{3}{*}{ cocaine $250 \mu \mathrm{M}$} & 1,3247 & 2948 \\
\hline & 1,3133 & 2617 \\
\hline & 1,3707 & 2713 \\
\hline \multirow{3}{*}{ benzoate $750 \mu \mathrm{M}$} & 1,2473 & 26246 \\
\hline & 1,3372 & 27386 \\
\hline & 1,3539 & 28481 \\
\hline \multirow{3}{*}{ cocaine $750 \mu \mathrm{M}$} & 1,3898 & 3679 \\
\hline & 1,3668 & 3425 \\
\hline & 1,3989 & 3409 \\
\hline \multirow{3}{*}{ benzoate $1000 \mu \mathrm{M}$} & 1,2397 & 27409 \\
\hline & 1,5418 & 31347 \\
\hline & 1,5089 & 31838 \\
\hline \multirow[b]{2}{*}{ cocaine $1000 \mu \mathrm{M}$} & 1,4615 & 4245 \\
\hline & $\begin{array}{l}1,5283 \\
1,4622\end{array}$ & 4844 \\
\hline
\end{tabular}

\begin{tabular}{|c|c|c|c|c|c|c|c|c|}
\hline & & no inducer & $10 \mu \mathrm{M}$ & $50 \mu \mathrm{M}$ & $100 \mathrm{\mu m}$ & $250 \mu \mathrm{M}$ & $700 \mu \mathrm{M}$ & $1000 \mathrm{\mu M}$ \\
\hline \multirow[t]{2}{*}{ means } & cocaine & \multirow{2}{*}{$\begin{array}{r}0 \\
0\end{array}$} & \multirow{2}{*}{$\begin{array}{l}838,166667 \\
3819,83333\end{array}$} & \multirow{2}{*}{$\begin{array}{r}507,166667 \\
8258,5\end{array}$} & \multirow{2}{*}{$\begin{array}{r}1006,5 \\
10642,166\end{array}$} & \multirow{2}{*}{$\begin{array}{l}883,833333 \\
23457,1667\end{array}$} & \multirow{2}{*}{$\begin{array}{r}1628,83333 \\
25495,5\end{array}$} & \multirow{2}{*}{$\begin{array}{r}2552,5 \\
28322,5\end{array}$} \\
\hline & benzoate & & & & & & & \\
\hline \multirow[t]{4}{*}{ std } & cocaine & & 433,481641 & \multirow{2}{*}{$\begin{array}{l}35,501173 \\
523,66496\end{array}$} & \multirow{2}{*}{$\begin{array}{l}406,18099 \\
585,35658\end{array}$} & \multirow{2}{*}{$\begin{array}{l}170,294842 \\
614,733547\end{array}$} & 151,477171 & \multirow{2}{*}{$\begin{array}{r}361,13294 \\
2427,78932\end{array}$} \\
\hline & benzoate & 625,207086 & 398,76977 & & & & 1117,5755 & \\
\hline & \multirow[t]{2}{*}{ RFP/OD } & & & & & & & \\
\hline & & no inducer & $10 \mu \mathrm{M}$ & $50 \mu \mathrm{M}$ & $100 \mu \mathrm{M}$ & $250 \mu \mathrm{M}$ & $700 \mu \mathrm{M}$ & $1000 \mu \mathrm{M}$ \\
\hline \multirow[t]{2}{*}{ means } & cocaine & \multirow{2}{*}{$\begin{array}{l}1438,23482 \\
1438,23482\end{array}$} & \multirow{2}{*}{$\begin{array}{l}1818,00292 \\
3885,68731\end{array}$} & \multirow{2}{*}{$\begin{array}{l}1721,40976 \\
6988,95117\end{array}$} & \multirow{2}{*}{$\begin{array}{l}1948,61900 \\
9263,74794\end{array}$} & \multirow{2}{*}{$\begin{array}{l}2065,79345 \\
17266,7087\end{array}$} & \multirow{2}{*}{$\begin{array}{l}2529,9704 \\
20852,8749\end{array}$} & \multirow{2}{*}{$\begin{array}{l}2981,0164 \mathrm{C} \\
21180,317\end{array}$} \\
\hline & benzoate & & & & & & & \\
\hline \multirow[t]{2}{*}{ std } & cocaine & \multirow{2}{*}{$\begin{array}{l}271,882912 \\
271,882912\end{array}$} & \multirow{2}{*}{$\begin{array}{l}226,57957 \\
127,95459\end{array}$} & \multirow{2}{*}{$\begin{array}{l}42,5471448 \\
203,44985\end{array}$} & \multirow{2}{*}{$\begin{array}{l}177,83618 \\
308,99393\end{array}$} & \multirow{2}{*}{$\begin{array}{l}138,39408 \\
604,671516\end{array}$} & \multirow{2}{*}{$\begin{array}{l}107,169338 \\
322,83970\end{array}$} & \multirow{2}{*}{$\begin{array}{r}164,22832 \\
891,682889\end{array}$} \\
\hline & benzoate & & & & & & & \\
\hline \multicolumn{9}{|c|}{ Id Change (RFP) } \\
\hline & & \begin{tabular}{|l|} 
no inducer \\
\end{tabular} & $10 \mu \mathrm{M}$ & $50 \mu \mathrm{M}$ & $100 \mu \mathrm{M}$ & $250 \mu \mathrm{M}$ & $700 \mu \mathrm{M}$ & $1000 \mu \mathrm{M}$ \\
\hline \multirow[t]{2}{*}{ means } & cocaine & & \multirow{2}{*}{$\begin{array}{l}1,44690305 \\
3,03670132\end{array}$} & 1,27041678 & 1,53665689 & 1,47125211 & 1,86847952 & 2,36097041 \\
\hline & benzoate & & & 5,4033591 & 6,67430907 & 13,5071536 & 14,5939749 & 16,1013063 \\
\hline std & & 0,33335488 & 0,23112857 & 0,01892891 & 0,21657211 & 0,0907997 & 0,08076629 & 0,19255289 \\
\hline & benzoate & 0,33335488 & 0,21262051 & 0,27921353 & 0,31210695 & 0,32777049 & 0,59588137 & 1,29447578 \\
\hline
\end{tabular}


BL21: HipO metabolic module + benzoate sensing module

\begin{tabular}{|c|c|c|}
\hline & & $\operatorname{RFP}$ (а.u.) \\
\hline \multirow{5}{*}{ no inducer } & 1,1227 & 48 \\
\hline & 0,5927 & 62 \\
\hline & 0,5479 & 69 \\
\hline & 0,5922 & 58 \\
\hline & 0,9875 & 60 \\
\hline \multirow{3}{*}{ hippurate $10 \mu \mathrm{M}$} & 0,8364 & 81 \\
\hline & 0,5812 & 92 \\
\hline & 0,6647 & 69 \\
\hline \multirow{3}{*}{ benzoate 10uM } & 0,5904 & 66 \\
\hline & 0,6113 & 72 \\
\hline & 0,6079 & 90 \\
\hline \multirow{3}{*}{ hippurate $25 \mu \mathrm{M}$} & 0,7916 & 160 \\
\hline & 0,6198 & 131 \\
\hline & 0,6074 & 107 \\
\hline \multirow{3}{*}{ benzoate $25 \mu \mathrm{M}$} & 0,5514 & 115 \\
\hline & 0,5568 & 85 \\
\hline & 0,6358 & 108 \\
\hline \multirow{3}{*}{ hippurate $50 \mu \mathrm{M}$} & 1,0303 & 224 \\
\hline & 0,5798 & 234 \\
\hline & 0,5782 & 236 \\
\hline \multirow{3}{*}{ benzoate $50 \mu \mathrm{M}$} & 0,5618 & 193 \\
\hline & 0,5615 & 205 \\
\hline & 0,6246 & 276 \\
\hline \multirow{3}{*}{ hippurate $100 \mu \mathrm{M}$} & 1,0341 & 391 \\
\hline & 0,5741 & 422 \\
\hline & 0,557 & 351 \\
\hline \multirow{3}{*}{ benzoate $100 \mu \mathrm{M}$} & 0,5551 & 501 \\
\hline & 0,5664 & 446 \\
\hline & 0,5789 & 428 \\
\hline \multirow{3}{*}{ hippurate $250 \mu \mathrm{M}$} & 0,4549 & 421 \\
\hline & 0,4853 & 459 \\
\hline & 0,5137 & 374 \\
\hline \multirow{3}{*}{ benzoate $250 \mu \mathrm{M}$} & 0,5125 & 497 \\
\hline & 0,5441 & 566 \\
\hline & 0,5933 & 494 \\
\hline \multirow{3}{*}{ 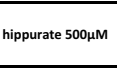 } & 0,2726 & 339 \\
\hline & 0,3041 & 379 \\
\hline & 0,3035 & 446 \\
\hline \multirow{3}{*}{ benzoate $500 \mu \mathrm{M}$} & 0,3741 & 485 \\
\hline & 0,4016 & 510 \\
\hline & 0,4538 & 512 \\
\hline \multirow{3}{*}{ hippurate $1000 \mu \mathrm{M}$} & 0,2635 & 273 \\
\hline & 0,3052 & 314 \\
\hline & 0,7672 & 467 \\
\hline \multirow{3}{*}{ benzoate $1000 \mu \mathrm{M}$} & 0,8103 & 701 \\
\hline & 0,6301 & 608 \\
\hline & 0,3564 & 433 \\
\hline
\end{tabular}

\begin{tabular}{|c|c|c|c|c|c|c|c|c|c|}
\hline & & \begin{tabular}{|l|} 
no inducer \\
\end{tabular} & $10 \mu \mathrm{M}$ & $25 \mu \mathrm{M}$ & $50 \mu \mathrm{M}$ & $100 \mu \mathrm{M}$ & $250 \mu \mathrm{M}$ & $500 \mu \mathrm{M}$ & $1000 \mu \mathrm{M}$ \\
\hline \multirow[t]{2}{*}{ means } & hippurate & & 21,2666667 & 73,2666667 & 171,933333 & 328,6 & 358,6 & 328,6 & 291,933333 \\
\hline & benzoate & 0 & 16,6 & 43,2666667 & 165,266667 & 398,933333 & 459,6 & 442,933333 & 521,266667 \\
\hline \multirow[t]{4}{*}{ std } & hippurate & 7,60263112 & 11,5036226 & 26,5392791 & 6,42910051 & 35,5949435 & 42,5793377 & 54,064776 & 102,246434 \\
\hline & benzoate & 7,60263112 & 12,489996 & 15,6950098 & 44,8590385 & 38,0306894 & 40,7308237 & 15,0443788 & 136,074734 \\
\hline & RFP/OD & & & & & & & & \\
\hline & & \begin{tabular}{|l|} 
no inducer \\
\end{tabular} & $10 \mu \mathrm{M}$ & $25 \mu \mathrm{M}$ & $50 \mu \mathrm{M}$ & $100 \mu \mathrm{M}$ & $250 \mu \mathrm{M}$ & $500 \mu \mathrm{M}$ & $1000 \mu \mathrm{M}$ \\
\hline \multirow[t]{2}{*}{ means } & hippurate & 86,3989767 & 119,647677 & 196,547157 & 343,054371 & 581,110575 & 866,445412 & 1319,80105 & 891,19789 \\
\hline & benzoate & 86,3989767 & 125,873687 & 177,027604 & 383,504977 & 809,76756 & 947,545699 & 1231,53848 & 1014,98831 \\
\hline \multirow[t]{2}{*}{ std } & hippurate & 33,875058 & 33,648567 & 18,2491952 & 108,833186 & 183,464105 & 120,282964 & 129,669492 & 244,670929 \\
\hline & benzoate & 33,875058 & 9 & 28,6310657 & 51,6926616 & 83,8651652 & 105,576413 & 90,4279764 & 180,200882 \\
\hline
\end{tabular}

\begin{tabular}{|c|c|c|c|c|c|c|c|c|c|}
\hline & & no inducer & $10 \mu \mathrm{M}$ & $25 \mu \mathrm{M}$ & $50 \mu \mathrm{M}$ & $100 \mu \mathrm{M}$ & $250 \mu \mathrm{M}$ & $500 \mu \mathrm{M}$ & $1000 \mu \mathrm{M}$ \\
\hline \multirow[t]{2}{*}{ means } & hippurate & & & 2,2334 & 3,89450056 & 6,5319 & 7,0370 & & \\
\hline & benzoate & & 1,27946128 & 1,72839506 & 3,78226712 & 7,71604938 & & & 9,7755 \\
\hline \multirow[t]{2}{*}{ std } & hippurate & 0,12799042 & 0,19366368 & 0,44678921 & 0,10823402 & 0,59924147 & 0,71682387 & 0,91018141 & 1,72132044 \\
\hline & benzoate & & 102692 & 2642257: & 0,75520267 & 0,6402473 & 0,685704 & 0,2532723 & 2,29082044 \\
\hline
\end{tabular}


BL21: benzoate sensing module

\begin{tabular}{|c|c|c|}
\hline \multirow{6}{*}{ no inducer } & 1,5831 & (a.u. \\
\hline & 1,3088 & 1808 \\
\hline & 1,2266 & 1585 \\
\hline & 1,1706 & 1659 \\
\hline & 1,2046 & 1579 \\
\hline & 1,1924 & 1489 \\
\hline \multirow{3}{*}{ benzoate $10 \mu \mathrm{M}$} & 1,4141 & 5320 \\
\hline & 1,5218 & 6114 \\
\hline & 1,4577 & 5652 \\
\hline \multirow{3}{*}{ hippurate $10 \mu \mathrm{M}$} & 1,4236 & 2163 \\
\hline & 1,3719 & 2012 \\
\hline & 1,4545 & \\
\hline \multirow{3}{*}{ benzoate $50 \mu \mathrm{M}$} & 1,4116 & 9534 \\
\hline & 1,477 & 10499 \\
\hline & 1,4595 & 10369 \\
\hline \multirow{3}{*}{ hippurate $50 \mu \mathrm{M}$} & 1,4236 & 2460 \\
\hline & 1,3719 & 3208 \\
\hline & 1,4545 & 2752 \\
\hline \multirow{3}{*}{ benzoate $100 \mu \mathrm{M}$} & 1,4037 & 13166 \\
\hline & 1,3494 & 12028 \\
\hline & 1,3012 & 12359 \\
\hline \multirow{3}{*}{ hippurate $100 \mu \mathrm{M}$} & 1,4935 & 3045 \\
\hline & 1,4474 & 2915 \\
\hline & 1,4972 & 3210 \\
\hline \multirow{3}{*}{ benzoate $250 \mu \mathrm{M}$} & 1,4125 & 25298 \\
\hline & 1,5113 & 25964 \\
\hline & 1,4803 & 24736 \\
\hline \multirow{3}{*}{ hippurate $250 \mu \mathrm{M}$} & 1,4575 & 3323 \\
\hline & 1,3115 & 2956 \\
\hline & 1,4401 & 3234 \\
\hline \multirow{3}{*}{ benzoate $750 \mu \mathrm{M}$} & 1,2473 & 26246 \\
\hline & 1,3372 & 27386 \\
\hline & 1,3539 & 28481 \\
\hline \multirow{3}{*}{ hippurate $750 \mu \mathrm{M}$} & 1,3079 & 3097 \\
\hline & 1,1987 & 2649 \\
\hline & 1,3313 & 3088 \\
\hline \multirow{3}{*}{ benzoate $1000 \mu \mathrm{M}$} & 1,2397 & 27409 \\
\hline & 1,5418 & 31347 \\
\hline & 1,5089 & 31838 \\
\hline \multirow{3}{*}{ hippurate $1000 \mu \mathrm{M}$} & 1,4514 & 3925 \\
\hline & 1,1243 & 2670 \\
\hline & 1,3936 & 3452 \\
\hline
\end{tabular}

\begin{tabular}{|c|c|c|c|c|c|c|c|c|}
\hline & & \begin{tabular}{|l|} 
no inducer \\
\end{tabular} & $10 \mu \mathrm{M}$ & $50 \mu \mathrm{M}$ & $100 \mu \mathrm{M}$ & $250 \mu \mathrm{M}$ & $700 \mu \mathrm{M}$ & $1000 \mu \mathrm{M}$ \\
\hline \multirow[t]{2}{*}{ means } & hippurate & & 280,166667 & 931,166667 & 1181,16667 & 1295,5 & 1069,16667 & 1473,5 \\
\hline & benzoate & 0 & 3819,83333 & 8258,5 & 10642,1667 & 23457,1667 & 25495,5 & 28322,5 \\
\hline \multirow[t]{4}{*}{ std } & hippurate & 625,207086 & 140,143974 & 376,984527 & 147,84564 & 191,439285 & 256,094384 & 633,808331 \\
\hline & benzoate & | 625,207086 & 398,769775 & 523,664969 & 585,356586 & 614,733547 & 1117,5755 & 2427,78932 \\
\hline & RFP/OD & & & & & & & \\
\hline & & \begin{tabular}{|l|} 
no inducer \\
\end{tabular} & $10 \mu \mathrm{M}$ & $50 \mu \mathrm{M}$ & $100 \mu \mathrm{M}$ & $250 \mu \mathrm{M}$ & $700 \mathrm{\mu M}$ & $1000 \mu \mathrm{M}$ \\
\hline \multirow[t]{2}{*}{ means } & hippurate & 1438,23482 & 1520,58864 & 1906,02289 & 2065,59772 & 2259,83884 & 2299,11646 & 2518,71147 \\
\hline & benzoate & 1438,23482 & 3885,68731 & 6988,95117 & 9263,74794 & 17266,7087 & 20852,8749 & 21180,3171 \\
\hline \multirow[t]{4}{*}{ std } & hippurate & 271,882912 & 54,6199301 & 161,412176 & 69,0302817 & 17,8806508 & 80,9669856 & 168,644243 \\
\hline & benzoate & 271,882912 & 127,954593 & 203,449854 & 308,993937 & 604,671516 & 322,839706 & 891,682889 \\
\hline & Change [ & & & & & & & \\
\hline & & \begin{tabular}{|l} 
no inducer \\
\end{tabular} & $10 \mu \mathrm{M}$ & $50 \mu \mathrm{M}$ & $100 \mu \mathrm{M}$ & $250 \mu \mathrm{M}$ & $700 \mu \mathrm{M}$ & $1000 \mu \mathrm{M}$ \\
\hline \multirow[t]{2}{*}{ means } & hippurate & & 1,14938239 & 1,49648982 & 1,62978761 & 1,69074913 & 1,570070 & 1,78565716 \\
\hline & & & 3,03670132 & 5,4033591 & 6,67430 & & & \\
\hline \multirow[t]{2}{*}{ std } & hippurate & 0,33335488 & 0,07472353 & 0,20100481 & 0,07882999 & 0,10207373 & 0,13654726 & 0,33794099 \\
\hline & benzoate & 0,33335488 & 0,21262051 & 0,27921353 & 0,31210695 & 0,32777049 & 0,59588137 & 1,29447578 \\
\hline
\end{tabular}


BL21: PTE-S5 metabolic module + 4-nitrophenol sensing module

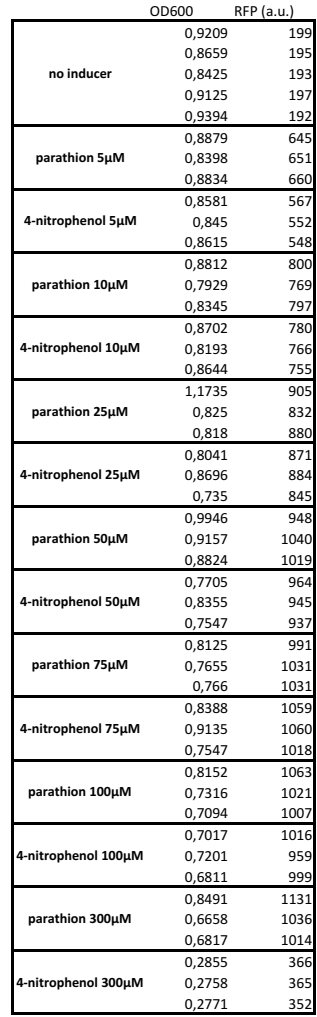

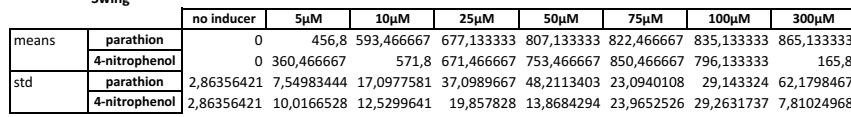

RFP/OD

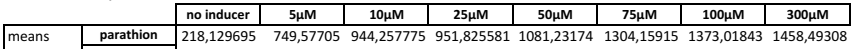

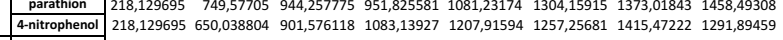

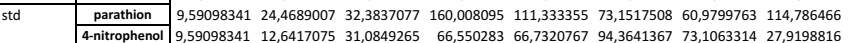

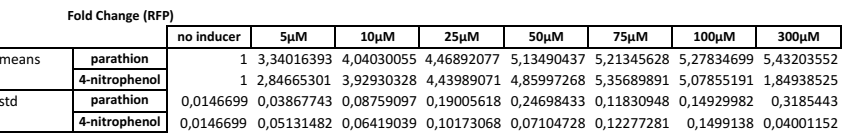


BL21: 4-nitrophenol sensing module

\begin{tabular}{|c|c|c|}
\hline & 600 & $\operatorname{RFP}$ (a.u.) \\
\hline \multirow{5}{*}{ no inducer } & 1,3601 & 154 \\
\hline & 1,0694 & 138 \\
\hline & 1,1037 & 140 \\
\hline & 1,1063 & 136 \\
\hline & 1,0639 & 137 \\
\hline \multirow[b]{2}{*}{ parathion $5 \mu \mathrm{M}$} & 1,0423 & 134 \\
\hline & 1,2693 & 149 \\
\hline \multirow{3}{*}{ 4-nitrophenol $5 \mu \mathrm{M}$} & $\frac{1,1364}{10241}$ & $\frac{142}{375}$ \\
\hline & 1,0667 & 375 \\
\hline & 1,0137 & 385 \\
\hline \multirow{3}{*}{ parathion $10 \mu \mathrm{M}$} & 1,0695 & 139 \\
\hline & 1,1688 & 149 \\
\hline & 1,1963 & 149 \\
\hline \multirow{3}{*}{ 4-nitrophenol 10 $10 \mathrm{M}$} & 1,0332 & 653 \\
\hline & 1,1364 & 667 \\
\hline & 1,0928 & 686 \\
\hline \multirow{3}{*}{ parathion $25 \mu \mathrm{M}$} & 1,1208 & 152 \\
\hline & 1,2083 & 167 \\
\hline & 1,202 & 161 \\
\hline \multirow{3}{*}{ 4-nitrophenol $25 \mu \mathrm{M}$} & 0,9572 & 1113 \\
\hline & 1,0074 & 1189 \\
\hline & 1,1033 & 1182 \\
\hline \multirow{3}{*}{ parathion $50 \mu \mathrm{M}$} & 1,096 & 167 \\
\hline & 1,224 & 191 \\
\hline & 1,047 & 168 \\
\hline \multirow{3}{*}{ 4-nitrophenol $50 \mu \mathrm{M}$} & 0,8965 & 1218 \\
\hline & 0,9659 & 1281 \\
\hline & 0,9212 & 1216 \\
\hline \multirow{3}{*}{ parathion $75 \mu \mathrm{M}$} & 1,0882 & 185 \\
\hline & 1,0088 & 198 \\
\hline & 1,0351 & 191 \\
\hline \multirow{3}{*}{ 4-nitrophenol 75 } & 0,9348 & 1224 \\
\hline & 0,8886 & 1241 \\
\hline & 0,8835 & 1234 \\
\hline \multirow{3}{*}{ parathion $100 \mu \mathrm{M}$} & 1,0131 & 216 \\
\hline & 1,0405 & 237 \\
\hline & 1,0423 & \\
\hline \multirow{3}{*}{ 4-nitrophenol $100 \mu \mathrm{M}$} & 0,7754 & 1138 \\
\hline & 0,7779 & 1140 \\
\hline & 0,8184 & 1191 \\
\hline \multirow{2}{*}{ parathion $300 \mu \mathrm{M}$} & 1,1479 & \\
\hline & 0,9214 & 305 \\
\hline \multirow{3}{*}{ 4-nitrophenol $300 \mu \mathrm{M}$} & $\begin{array}{l}0,9113 \\
0,4667\end{array}$ & 538 \\
\hline & 0,4732 & \\
\hline & & \\
\hline
\end{tabular}

\begin{tabular}{|c|c|c|c|c|c|c|c|c|c|}
\hline & & \begin{tabular}{|l|} 
no inducer \\
\end{tabular} & 5нM & \begin{tabular}{|c|}
$10 \mu \mathrm{M}$ \\
\end{tabular} & $25 \mu \mathrm{M}$ & $50 \mu \mathrm{M}$ & $75 \mu \mathrm{M}$ & $100 \mu \mathrm{M}$ & $300 \mu \mathrm{M}$ \\
\hline \multirow[t]{2}{*}{ means } & \begin{tabular}{|l|} 
parathion \\
\end{tabular} & & 0,66666667 & 4,66666667 & 19 & 34,3333333 & 50,3333333 & 85,3333333 & 148,666667 \\
\hline & 4-nitrophenol & & 237,333333 & 527,666667 & 1020,33333 & 1097,33333 & 1092 & 1015,33333 & 384,333333 \\
\hline \multirow[t]{4}{*}{ std } & \begin{tabular}{|l|} 
parathion \\
\end{tabular} & 7,41619849 & 7,5055535 & 5,77350269 & 7,54983444 & 13,5769412 & 6,5064071 & 10,5039675 & 17,8978583 \\
\hline & 4-nitrophenol & 7,41619849 & 5,77350269 & 16,563011 & 42,0039681 & 36,9639464 & 8,54400375 & 30,0388637 & 13,0128142 \\
\hline & RFP/OD & & & & & & & & \\
\hline & & no inducer & $5 \mu \mathrm{M}$ & $10 \mu \mathrm{M}$ & $25 \mu \mathrm{M}$ & $50 \mu \mathrm{M}$ & $75 \mu \mathrm{M}$ & $100 \mu \mathrm{M}$ & $300 \mathrm{MM}$ \\
\hline \multirow[t]{2}{*}{ means } & \begin{tabular}{|l|} 
parathion \\
\end{tabular} & \begin{tabular}{|l|l}
124,164231 \\
\end{tabular} & 123,635124 & 127,33305 & 135,923851 & 156,292156 & 183,600516 & 219,270088 & 289,639088 \\
\hline & 4-nitrophenol & 124,164231 & 365,841159 & 615,567831 & 1138,1213 & 1334,95282 & 1367,55583 & 1462,7974 & 1115,29778 \\
\hline \multirow[t]{4}{*}{ std } & \begin{tabular}{|l|} 
parathion \\
\end{tabular} & 6,58361297 & 5,70304931 & 2,71132465 & 2,15008137 & 4,04872242 & 13,1579304 & 7,58483732 & 49,2176847 \\
\hline & 4-nitrophenol & 6,58361297 & 14,1255972 & 24,8832132 & 58,4997527 & 20,7272972 & 50,3895949 & 6,59926213 & 35,7636625 \\
\hline & Id Change (F) & & & & & & & & \\
\hline & & no inducer & $5 \mu \mathrm{M}$ & $10 \mu \mathrm{M}$ & $25 \mu \mathrm{M}$ & $50 \mu \mathrm{M}$ & $75 \mu \mathrm{M}$ & $100 \mu \mathrm{M}$ & $300 \mu \mathrm{M}$ \\
\hline \multirow[t]{2}{*}{ means } & \begin{tabular}{|l|} 
parathion \\
\end{tabular} & & & & 1,13475177 & 1,24349882 & & & 2,05437352 \\
\hline & & & 2,68321513 & 4,74231678 & 8,23640662 & 8,78250591 & 8,74468085 & 8,20094563 & 3,72576832 \\
\hline \multirow[t]{2}{*}{ std } & parathion & 0,05259715 & 0,05323088 & 0,04094683 & 0,05354493 & 0,09629036 & 0,04614473 & 0,07449622 & 0,12693517 \\
\hline & 4-nitrophenol & & & & 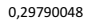 & & 506059577 & 0,21304159 & 0,09228946 \\
\hline
\end{tabular}


BL21: pnpA-pnpB metabolic module + chlorohydroquinone sensing module

\begin{tabular}{|c|c|c|}
\hline & & RFP (a.u.) \\
\hline \multirow{8}{*}{ no inducer } & 0,9341 & 45 \\
\hline & 0,8173 & 52 \\
\hline & 0,6816 & 48 \\
\hline & 0,9378 & 48 \\
\hline & 0,8174 & 49 \\
\hline & 0,7037 & 50 \\
\hline & 0,9205 & 46 \\
\hline & 0,8026 & 49 \\
\hline \multirow{6}{*}{ 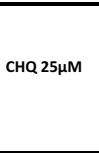 } & 0,9263 & 56 \\
\hline & 0,768 & 56 \\
\hline & 0,7998 & 56 \\
\hline & 0,9909 & 52 \\
\hline & 0,7835 & 52 \\
\hline & 0,8934 & 55 \\
\hline \multirow{6}{*}{ 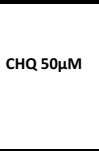 } & 1,1537 & 63 \\
\hline & 0,8759 & 62 \\
\hline & 0,836 & 62 \\
\hline & 0,7145 & 63 \\
\hline & 0,4871 & 67 \\
\hline & 0,9783 & 60 \\
\hline \multirow{6}{*}{ CHQ $75 \mu \mathrm{M}$} & 0,655 & 69 \\
\hline & 0,6241 & 69 \\
\hline & 0,8477 & 67 \\
\hline & 0,6917 & 71 \\
\hline & 0,5746 & 71 \\
\hline & 0,8311 & 70 \\
\hline \multirow{8}{*}{ CHQ $100 \mu \mathrm{M}$} & 0,6543 & 75 \\
\hline & 0,9184 & 70 \\
\hline & 0,6633 & 73 \\
\hline & 0,7292 & 72 \\
\hline & 1,1171 & 67 \\
\hline & 0,7541 & 67 \\
\hline & 0,8595 & 70 \\
\hline & 0,6251 & 73 \\
\hline \multirow{6}{*}{ 2C4NP 25 $\mu \mathrm{M}$} & 0,8719 & 54 \\
\hline & 0,6908 & 57 \\
\hline & 0,801 & 54 \\
\hline & 1,1307 & 52 \\
\hline & 1,0324 & 52 \\
\hline & 0,8898 & 50 \\
\hline \multirow{6}{*}{ 2C4NP 50 $\mu \mathrm{M}$} & 0,5751 & 66 \\
\hline & 0,688 & 62 \\
\hline & 0,7809 & 63 \\
\hline & 0,6178 & 65 \\
\hline & 1,1442 & 60 \\
\hline & 0,8566 & 59 \\
\hline \multirow{6}{*}{2 C4NP $75 \mu \mathrm{M}$} & 0,4794 & 71 \\
\hline & 0,5387 & 69 \\
\hline & 0,598 & 68 \\
\hline & 0,6853 & 67 \\
\hline & 0,7204 & 65 \\
\hline & 0,9524 & 63 \\
\hline \multirow{8}{*}{2 C4NP $100 \mu \mathrm{M}$} & 0,6272 & 71 \\
\hline & 0,637 & 67 \\
\hline & 0,5032 & 72 \\
\hline & 0,5614 & 72 \\
\hline & 0,4618 & 73 \\
\hline & 0,5134 & 70 \\
\hline & 0,5133 & 71 \\
\hline & 0,7321 & 68 \\
\hline
\end{tabular}

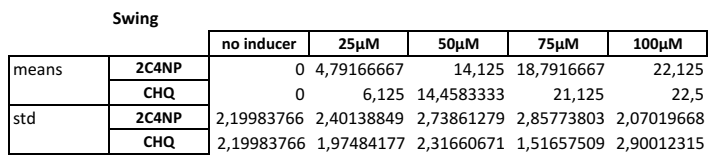

RFP/OD

\begin{tabular}{|c|c|c|c|c|c|c|}
\hline \multirow{3}{*}{ means } & & no inducer & $25 \mu \mathrm{M}$ & $50 \mu \mathrm{M}$ & $75 \mu \mathrm{M}$ & $100 \mu \mathrm{M}$ \\
\hline & 2 C4NP & 59,4285747 & 60,735359 & 85,3470758 & 107,340672 & 126,918038 \\
\hline & $\mathrm{CHQ}$ & 59,4285747 & 63,9664534 & 81,1011864 & 100,895952 & 93,336041 \\
\hline \multirow[t]{2}{*}{ std } & 2C4NP & 8,94857402 & 13,1597738 & 23,0594997 & 29,0300715 & 21,6198555 \\
\hline & CHQ & 8,94857402 & 7,38367116 & 29,9457451 & 16,6479067 & 20,2805616 \\
\hline
\end{tabular}

Fold Change (RFP)

\begin{tabular}{|c|c|c|c|c|c|c|}
\hline \multicolumn{7}{|c|}{ Fold Change (RFP) } \\
\hline & & no inducer & $25 \mu \mathrm{M}$ & $50 \mu \mathrm{M}$ & $75 \mu \mathrm{M}$ & $100 \mu \mathrm{M}$ \\
\hline \multirow[t]{2}{*}{ means } & 2 CANP & 1 & 1,09905254 & 1,29198966 & 1,38845823 & 1,45736434 \\
\hline & $\mathrm{CHQ}$ & 1 & 1,12661499 & 1,29888028 & 1,43669251 & 1,46511628 \\
\hline \multirow[t]{2}{*}{ std } & 2C4NP & 0,04547468 & 0,04964111 & 0,05661215 & 0,05907469 & 0,04279476 \\
\hline & $\mathrm{CHQ}$ & 0,04547468 & 0,0408236 & 0,04788851 & 0,03135039 & 0,05995087 \\
\hline
\end{tabular}


BL21: chlorohydroquinone sensing module

\begin{tabular}{|c|c|c|}
\hline & & RFP (a.u.) \\
\hline \multirow{10}{*}{ no inducer } & 1,5331 & 63 \\
\hline & 1,4154 & 59 \\
\hline & 1,4077 & 59 \\
\hline & 1,4106 & 60 \\
\hline & 1,3615 & 57 \\
\hline & 1,4511 & 60 \\
\hline & 1,405 & 59 \\
\hline & 1,3897 & 59 \\
\hline & 1,4127 & 62 \\
\hline & 1,3933 & 58 \\
\hline \multirow{5}{*}{ 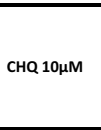 } & 1,4331 & 76 \\
\hline & 1,424 & 76 \\
\hline & 1,4014 & 75 \\
\hline & 1,2549 & 77 \\
\hline & 1,3243 & 76 \\
\hline \multirow{5}{*}{ 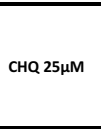 } & 1,3803 & 121 \\
\hline & 1,0076 & 118 \\
\hline & 1,2276 & 122 \\
\hline & 1,375 & 125 \\
\hline & 1,3974 & 131 \\
\hline \multirow{5}{*}{ 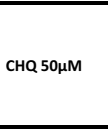 } & 1,3757 & 142 \\
\hline & 1,4003 & 144 \\
\hline & 1,1595 & 135 \\
\hline & 1,2744 & 136 \\
\hline & 1,4072 & 150 \\
\hline \multirow{5}{*}{ CHQ $75 \mu \mathrm{M}$} & 1,3244 & 141 \\
\hline & 1,1508 & 145 \\
\hline & 1,2165 & 149 \\
\hline & 1,1128 & 148 \\
\hline & 1,317 & 150 \\
\hline \multirow{5}{*}{ CHQ $100 \mu \mathrm{M}$} & 1,4318 & 159 \\
\hline & 1,3747 & 160 \\
\hline & 1,2495 & 154 \\
\hline & 1,2848 & 156 \\
\hline & 1,397 & 168 \\
\hline \multirow{5}{*}{ 2C4NP 10 $10 \mathrm{M}$} & 1,1904 & 57 \\
\hline & 1,237 & 57 \\
\hline & 1,1368 & 56 \\
\hline & 1,1601 & 58 \\
\hline & 0,9038 & 52 \\
\hline \multirow{5}{*}{ 2C4NP $25 \mu \mathrm{M}$} & 0,5446 & 56 \\
\hline & 1,213 & 55 \\
\hline & 0,8021 & 57 \\
\hline & 0,8362 & 55 \\
\hline & 1,2482 & 57 \\
\hline \multirow{5}{*}{ 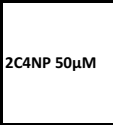 } & 1,009 & 59 \\
\hline & 0,5931 & 54 \\
\hline & 0,8817 & 55 \\
\hline & 0,8554 & 56 \\
\hline & 0,924 & 55 \\
\hline \multirow{5}{*}{2 C4NP $75 \mu \mathrm{M}$} & 0,9003 & 50 \\
\hline & 0,7662 & 52 \\
\hline & 0,7063 & 49 \\
\hline & 0,7596 & 49 \\
\hline & 0,8224 & 50 \\
\hline \multirow{5}{*}{$2 \mathrm{C} 4 \mathrm{NP} 100 \mu \mathrm{M}$} & 0,2468 & 71 \\
\hline & 0,2705 & 72 \\
\hline & 0,2774 & 70 \\
\hline & 0,2923 & 69 \\
\hline & 0,3816 & 66 \\
\hline
\end{tabular}

\begin{tabular}{|c|c|c|c|c|c|c|c|}
\hline \multirow{2}{*}{\multicolumn{2}{|c|}{ Swing }} & \multirow{2}{*}{\multicolumn{2}{|c|}{\begin{tabular}{l|l}
\multicolumn{2}{|l}{} \\
no inducer & $10 \mu \mathrm{M}$ \\
\end{tabular}}} & & & \\
\hline & & & & $25 \mu \mathrm{M}$ & $50 \mu \mathrm{M}$ & $75 \mu \mathrm{M}$ & $100 \mu \mathrm{M}$ \\
\hline \multirow[t]{2}{*}{ means } & 2C4NP & 0 & $-3,6$ & $-3,6$ & $-3,8$ & $-9,6$ & 10 \\
\hline & $\mathrm{CHQ}$ & 0 & 16,4 & 63,8 & 81,8 & 87 & 99,8 \\
\hline \multirow[t]{4}{*}{ std } & 2C4NP & 1,77638835 & 2,34520788 & 1 & 1,92353841 & 1,22474487 & 2,30217289 \\
\hline & $\mathrm{CHQ}$ & 1,77638835 & 0,70710678 & 4,92950302 & 6,14817046 & 3,64691651 & 5,36656315 \\
\hline & RFP/OD & & & & & & \\
\hline & & no inducer & $10 \mu \mathrm{M}$ & $25 \mu \mathrm{M}$ & $50 \mu \mathrm{M}$ & $75 \mu \mathrm{M}$ & $100 \mu \mathrm{M}$ \\
\hline \multirow[t]{2}{*}{ means } & 2C4NP & 42,0401972 & 50,150783 & 66,1345692 & 67,3781059 & 63,6170722 & 243,042818 \\
\hline & $\mathrm{CHQ}$ & 42,0401972 & 55,7337732 & 97,7615182 & 107,159265 & 120,36764 & 118,472946 \\
\hline \multirow[t]{2}{*}{ std } & 2C4NP & 0,78540957 & 4,38919829 & 23,5696442 & 13,507746 & 5,59587255 & 43,5177115 \\
\hline & $\mathrm{CHQ}$ & 0,78540957 & 3,6118399 & 11,6411653 & 5,49241294 & 10,3794662 & 4,8512717 \\
\hline
\end{tabular}

Fold Change (RFP)

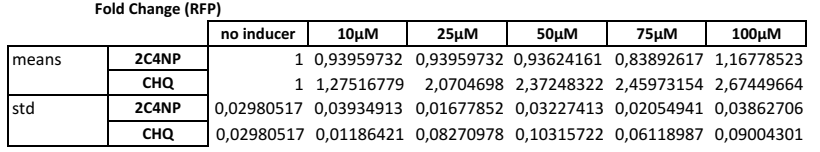


BL21: nitrite sensing module

\begin{tabular}{|c|c|c|}
\hline \multirow{4}{*}{ no inducer } & 10892 & RFP(a.u.) \\
\hline & 1,0891 & 3883 \\
\hline & 1,0903 & 3979 \\
\hline & 1,0743 & 3932 \\
\hline \multirow{4}{*}{ nitroglycerin $50 \mu \mathrm{M}$} & 1,0647 & 5306 \\
\hline & 1,084 & 5474 \\
\hline & 1,0833 & 5624 \\
\hline & 1,1203 & 5726 \\
\hline
\end{tabular}

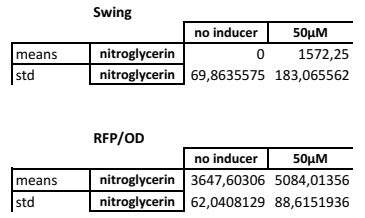

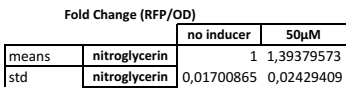

JW1642: nitrite sensing module

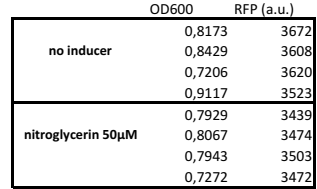

\begin{tabular}{|c|c|c|c|}
\hline \multirow{3}{*}{\begin{tabular}{|l} 
means \\
std
\end{tabular}} & Swing & \multicolumn{2}{|c|}{\begin{tabular}{|l|l} 
no inducer & $50 \mu \mathrm{M}$ \\
\end{tabular}} \\
\hline & nitroglycerin & 0 & $-133,75$ \\
\hline & nitroglycerin & 61,7650117 & 26,1661359 \\
\hline & RFP/OD & & \\
\hline & & no inducer & $50 \mu \mathrm{M}$ \\
\hline means & nitroglycerin & 4415,27594 & 4457,08164 \\
\hline
\end{tabular}

Fold Change (RFP/OD)
\begin{tabular}{|l|r|r|r|}
\hline \multicolumn{2}{|c|}{ no inducer } & $50 \mu \mathrm{MM}$ \\
\hline means & nitroglycerin & 1 & 1,00946842 \\
\hline std & nitroglycerin & 0,10924016 & 0,04892608
\end{tabular}

JW1642: NemA metabolic module + nitrite sensing module

\begin{tabular}{|c|c|c|}
\hline & 600 & $\operatorname{RFP}($ a.u. $)$ \\
\hline \multirow{4}{*}{ no inducer } & 0,3858 & 1963 \\
\hline & 0,4559 & 1996 \\
\hline & 0,4235 & 1945 \\
\hline & 0,4224 & 1939 \\
\hline \multirow{3}{*}{ nitroglycerin $50 \mu \mathrm{M}$} & 0,3727 & 2112 \\
\hline & 0,3688 & 2151 \\
\hline & $\begin{array}{r}0,369 \\
0,3609\end{array}$ & 2160 \\
\hline
\end{tabular}

\begin{tabular}{|c|c|c|c|}
\hline \multirow{2}{*}{\multicolumn{2}{|c|}{ Swing }} & \multirow{3}{*}{$\begin{array}{ll}\text { no inducer } \\
\end{array}$} & \multirow[b]{2}{*}{$50 \mu \mathrm{M}$} \\
\hline & & & \\
\hline means & \begin{tabular}{|l|} 
nitroglycerin \\
\end{tabular} & & 183,75 \\
\hline \multirow{3}{*}{ std } & \begin{tabular}{|l|} 
nitroglycerin \\
\end{tabular} & 25,6173769 & 21,977261 \\
\hline & RFP/OD & & \\
\hline & & \begin{tabular}{l|l|} 
no inducer \\
\end{tabular} & $50 \mu \mathrm{M}$ \\
\hline means & \begin{tabular}{|l|} 
nitroglycerin \\
\end{tabular} & 4662,34933 & 5831,00682 \\
\hline \multirow[t]{3}{*}{ std } & nitroglycerin & 301,153806 & 125,356593 \\
\hline & Id Change (RFP/C & D) & \\
\hline & & \begin{tabular}{|l|} 
no inducer \\
\end{tabular} & $50 \mu \mathrm{M}$ \\
\hline means & nitroglycerin & & 1,2506585 \\
\hline std & $\begin{array}{l}\text { nitroglycerin } \\
\end{array}$ & 0,06459272 & 0,026887 \\
\hline
\end{tabular}


Data file S2. sequences of plasmids used in this study

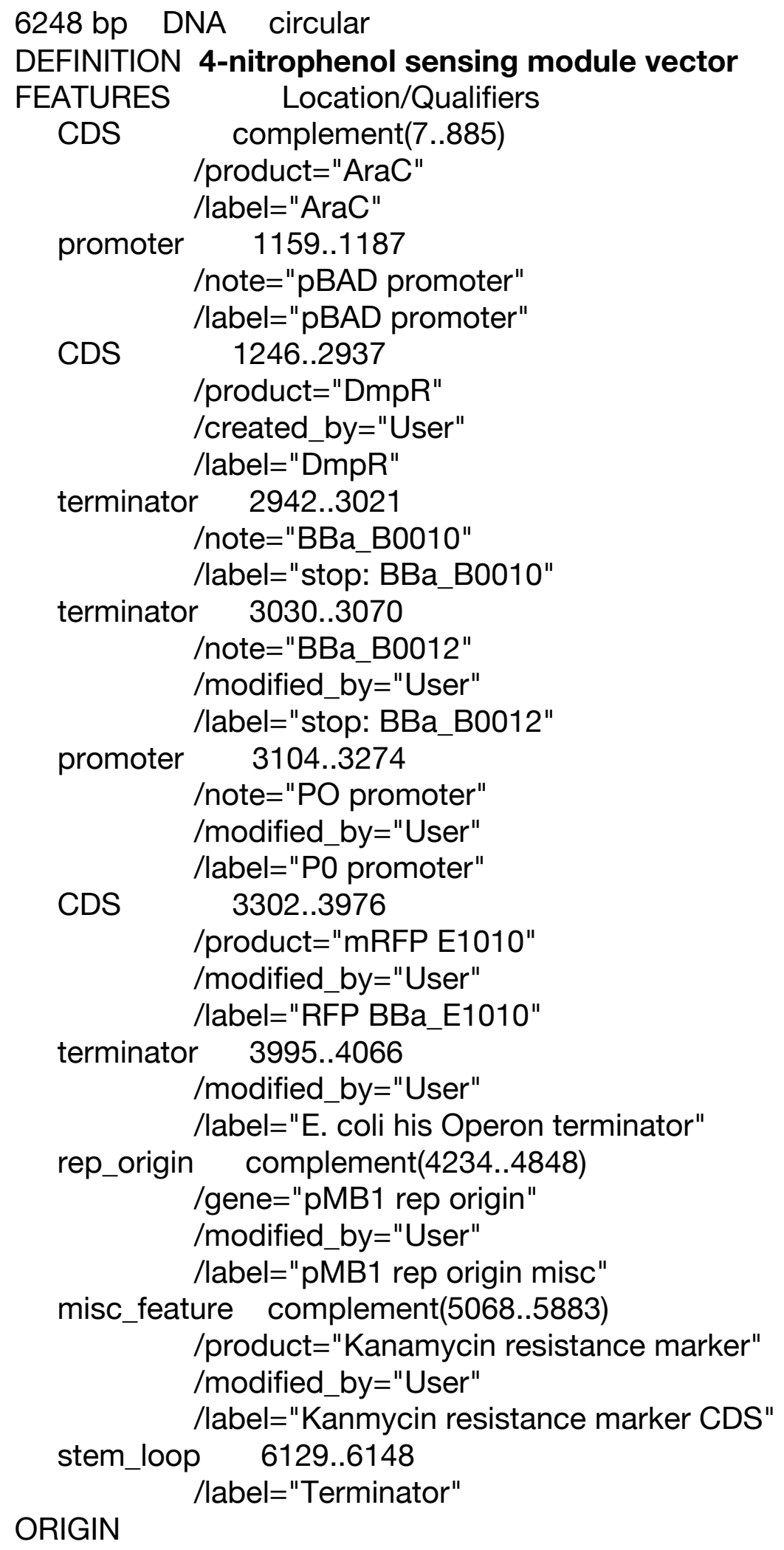


1 gacgtcttat gacaacttga cggctacatc attcactttt tcttcacaac cggcacggaa

61 ctcgctcggg ctggccccgg tgcattttt aaatacccgc gagaaataga gttgatcgtc

121 aaaaccaaca ttgcgaccga cggtggcgat aggcatccgg gtggtgctca aaagcagctt

181 cgcctggctg atacgttggt cctcgcgcca gcttaagacg ctaatcccta actgctggcg

241 gaaaagatgt gacagacgcg acggcgacaa gcaaacatgc tgtgcgacgc tggcgatatc

301 aaaattgctg tctgccaggt gatcgctgat gtactgacaa gcctcgcgta cccgattatc

361 catcggtgga tggagcgact cgttaatcgc ttccatgcgc cgcagtaaca attgctcaag

421 cagatttatc gccagcagct ccgaatagcg cccttcccct tgcccggcgt taatgatttg

481 cccaaacagg tcgctgaaat gcggctggtg cgcttcatcc gggcgaaaga accccgtatt

541 ggcaaatatt gacggccagt taagccattc atgccagtag gcgcgcggac gaaagtaaac

601 ccactggtga taccattcgc gagcctccgg atgacgaccg tagtgatgaa tctctcctgg

661 cgggaacagc aaaatatcac ccggtcggca aacaaattct cgtccctgat tttcaccac

721 cccctgaccg cgaatggtga gattgagaat ataacctttc attcccagcg gtcggtcgat

781 aaaaaaatcg agataaccgt tggcctcaat cggcgttaaa cccgccacca gatgggcatt

841 aaacgagtat cccggcagca ggggatcatt ttgcgettca gccatacttt tcatactccc

901 gccattcaga gaagaaacca attgtccata ttgcatcaga cattgccgtc actgcgtctt

961 ttactggctc ttctcgctaa ccaaaccggt aaccccgctt attaaaagca ttctgtaaca

1021 aagcgggacc aaagccatga caaaaacgcg taacaaaagt gtctataatc acggcagaaa

1081 agtccacatt gattatttgc acggcgtcac actttgctat gccatagcat ttttatccat

1141 aagattagcg gattctacct gacgctttt atcgcaactc tctactgttt ctccataccc

1201 gttttttgg gaattcaaaa gatcttttaa gaaggagata tacagatgcc gatcaagtac

1261 aagcctgaaa tccagcactc cgatttcaag gacctgacca acctgatcca cttccagagc

1321 atggaaggca agatctggct tggcgaacag cgcatgctgt tgcttcagtt ttcagcgatg

1381 gccagctttc gccgggaaat ggtcaatacc ctgggcatcg aacgcgccaa gggcttgttc

1441 ctgcgccatg gttaccagtc cggcctgaag gatgccgaac tggccaggaa gctgagaccg

1501 aatgccagcg aagtcggcat gttcctcgct gggccgcaga tgcattcact caagggtctg

1561 gtcaaggtcc gccccaccga gctcgatatc gacaaggaat acgggcgctt ctatgccgag

1621 atggagtgga tcgactcctt cgaggtggaa atctgccaga ccgacctggg gcagatgcaa

1681 gacccggtgt gctggactct gctcggctac gcctgcgcct attcctcggc gttcatgggc

1741 cgggaaatca tcttcaagga agtcagctgc ggcggctgcg gcggcgacaa gtgccgggtc

1801 attggcaagc cggccgaaga gtgggacgac gttgccagct tcaaacagta ttcaagaac

1861 gacccatca tcgaggaact ctacgagttg caatcgcaac tgttgtcgct gcgtaccaac

1921 ctcgacaaac aggaaggcca gtactacggc atcggtcaga ccccggccta ccagaccgtg

1981 cgcaatatga tggacaaggc cgcacagggc aaagtctcgg tgctgctgct tggcgagacc

2041 ggggtcggca aggaggtcat cgcgcgtagc gtgcacctgc gcagcaaacg cgccgccgag

2101 ccctttgtcg cggtgaactg tgcggcgatc ccgccggacc tgatcgagtc cgaattgttc

2161 ggcgtggaaa aaggcgcctt caccggcgcc tcccagtcac gcatgggccg cttcgagcgg

2221 gccgacaagg gcaccatctt ccttgacgag gtgatcgaac tcagcccgcg cgctcaggcc

2281 agtctgctgc gcgtgctgca agaaggcgag ctggagcgag ttggcgacaa ccgcacgcgc

2341 aagatcgacg taagggttat cgccgccacc cacgaggacc tggccgaagc ggtcaaggcc

2401 gggcgttttc gcgccgacct gtactaccga ctgaacgttt tcccggtggc gatcccggcg

$2461 \mathrm{ttg} g c g a a c$ gccgcgagga cattccactg ctggttgagc acttccttca gcgcttccac

2521 caggagtacg gcaagagaac cctcggcctt tcagacaaag ccctggaggc ctgcctgcat

2581 tacagttggc cgggcaatat ccgtgagctg gagaacgtca tcgagcgcgg catcatcctc

2641 accgatccga acgaaagcat cagcgtgcag gcgctgttcc tacgggcgcc ggaagagccg

2701 cagaccgcca gcgagcgggt gtcgttggac ggcgtgctga ttcagccagg caatggccag

2761 ggcagttgga tcagccagtt gttgagcagc ggcctgagcc tcgacgagat cgaggaaagc

2821 ctgatgcgcg aagccatgca acaggccaac caaaacgtct ccggtgccgc gcgcttgctc 
2881 ggcctaagcc gaccggcact ggcctatcgg ctgaagaaaa tcggcatcga aggctgaaga 2941 cccaggcatc aaataaaacg aaaggctcag tcgaaagact gggcctttcg ttttatctgt 3001 tgtttgtcgg tgaacgctct ctactagagt cacactggct caccttcggg tgggcctttc 3061 tgcgtttata tactagttgc ggaattcgcg gccgcttcta gagtaagcat ttgctcaagc 3121 ggccttgggc aattgatcaa atgcttaaaa agtctgcgca agcgcggctt aatttcgctc 3181 gctccgatca ttctaaaaat tagaaacaca ttgaaaaaca ttaccttgaa gtctgttttc 3241 agaccttggc acagccgttg cttgatgtcc tgcgtactag agtcacacag gaaagactag 3301 aatggettcc tccgaagacg ttatcaaaga gttcatgcgt ttcaaagttc gtatggaagg 3361 ttccgttaac ggtcacgagt tcgaaatcga aggtgaaggt gaaggtcgtc cgtacgaagg 3421 tacccagacc gctaaactga aagttaccaa aggtggtccg ctgccgttcg cttgggacat 3481 cctgtccccg cagttccagt acggttccaa agcttacgtt aaacacccgg ctgacatccc 3541 ggactacctg aaactgtcct tcccggaagg tttcaaatgg gaacgtgtta tgaacttcga 3601 agacggtggt gttgttaccg ttacccagga ctcctccctg caagacggtg agttcatcta 3661 caaagttaaa ctgcgtggta ccaacttccc gtccgacggt ccggttatgc agaaaaaaac 3721 catgggttgg gaagcttcca ccgaacgtat gtacccggaa gacggtgctc tgaaaggtga 3781 aatcaaaatg cgtctgaaac tgaaagacgg tggtcactac gacgctgaag ttaaaaccac 3841 ctacatggct aaaaaaccgg ttcagctgcc gggtgcttac aaaaccgaca tcaaactgga 3901 catcacctcc cacaacgaag actacaccat cgttgaacag tacgaacgtg ctgaaggtcg 3961 tcactccacc ggtgcttaat gcgtcttcct gcagtccggc aaaaaagggc aaggtgtcac 4021 caccctgccc tttttcttta aaaccgaaaa gattacttcg cgttatgcag gcttcctcgc 4081 tcactgactc gctgcgctcg gtcgttcggc tgcggcgagc ggtatcagct cactcaaagg 4141 cggtaatacg gttatccaca gaatcagggg ataacgcagg aaagaacatg tgagcaaaag 4201 gccagcaaaa ggccaggaac cgtaaaaagg ccgcgttgct ggcgttttc cacaggctcc 4261 gcccccctga cgagcatcac aaaaatcgac gctcaagtca gaggtggcga aacccgacag 4321 gactataaag ataccaggcg tttccccctg gaagctccct cgtgcgetct cctgttccga 4381 ccctgccgct taccggatac ctgtccgcet ttctccettc gggaagcgtg gcgetttctc 4441 atagctcacg ctgtaggtat ctcagttcgg tgtaggtcgt tcgctccaag ctgggctgtg 4501 tgcacgaacc ccccgttcag cccgaccgct gcgccttatc cggtaactat cgtcttgagt 4561 ccaacccggt aagacacgac ttatcgccac tggcagcagc cactggtaac aggattagca 4621 gagcgaggta tgtaggcggt gctacagagt tcttgaagtg gtggcctaac tacggctaca 4681 ctagaagaac agtatttggt atctgcgctc tgctgaagcc agttaccttc ggaaaaagag 4741 ttggtagctc ttgatccggc aaacaaacca ccgctggtag cggtggtttt tttgtttgca 4801 agcagcagat tacgcgcaga aaaaaaggat ctcaagaaga tcctttgatc ttttctacgg 4861 ggtctgacgc tcagtggaac gaaaactcac gttaagggat tttggtcatg agattatcaa 4921 aaaggatctt cacctagatc cttttaaatt aaaaatgaag ttttaaatca atctaaagta 4981 tatatgagta aacttggtct gacagctcga gtcccgtcaa gtcagcgtaa tgctctgcca 5041 gtgttacaac caattaacca attctgatta gaaaaactca tcgagcatca aatgaaactg 5101 caatttattc atatcaggat tatcaatacc atattttga aaaagccgtt tctgtaatga 5161 aggagaaaac tcaccgaggc agttccatag gatggcaaga tcctggtatc ggtctgcgat 5221 tccgactcgt ccaacatcaa tacaacctat taatttcccc tcgtcaaaaa taaggttatc 5281 aagtgagaaa tcaccatgag tgacgactga atccggtgag aatggcaaaa gcttatgcat 5341 ttctttccag acttgttcaa caggccagcc attacgctcg tcatcaaaat cactcgcatc 5401 aaccaaaccg ttattcattc gtgattgcgc ctgagcgaga cgaaatacgc gatcgctgtt 5461 aaaaggacaa ttacaaacag gaatcgaatg caaccggcgc aggaacactg ccagcgcatc 5521 aacaatattt tcacctgaat caggatattc ttctaatacc tggaatgctg ttttcccggg 5581 gatcgcagtg gtgagtaacc atgcatcatc aggagtacgg ataaaatgct tgatggtcgg 5641 aagaggcata aattccgtca gccagtttag tctgaccatc tcatctgtaa catcattggc 5701 aacgctacct ttgccatgtt tcagaaacaa ctctggcgca tcgggcttcc catacaatcg 
5761 atagattgtc gcacctgatt geccgacatt atcgcgagcc catttatacc catataaatc 5821 agcatccatg ttggaattta atcgcggcct ggagcaagac gtttcccgtt gaatatggct 5881 cataacaccc cttgtattac tgtttatgta agcagacagt tttattgttc atgatgatat 5941 attttatct tgtgcaatgt aacatcagag attttgagac acaacgtggc tttgttgaat 6001 aaatcgaact tttgctgagt tgaaggatca gctcgagtgc cacctgacgt ctaagaaacc 6061 attattatca tgacattaac ctataaaaat aggcgtatca cgaggcagaa tttcagataa 6121 aaaaaatcct tagctttcgc taaggatgat ttctggaatt cgaagagcct cggtagtgat $6181 \mathrm{cttatttcat}$ tatggtgaaa gttggaacct cttacgtgcc gatcaacgtc tcattttcgc // 6241 cagatatc

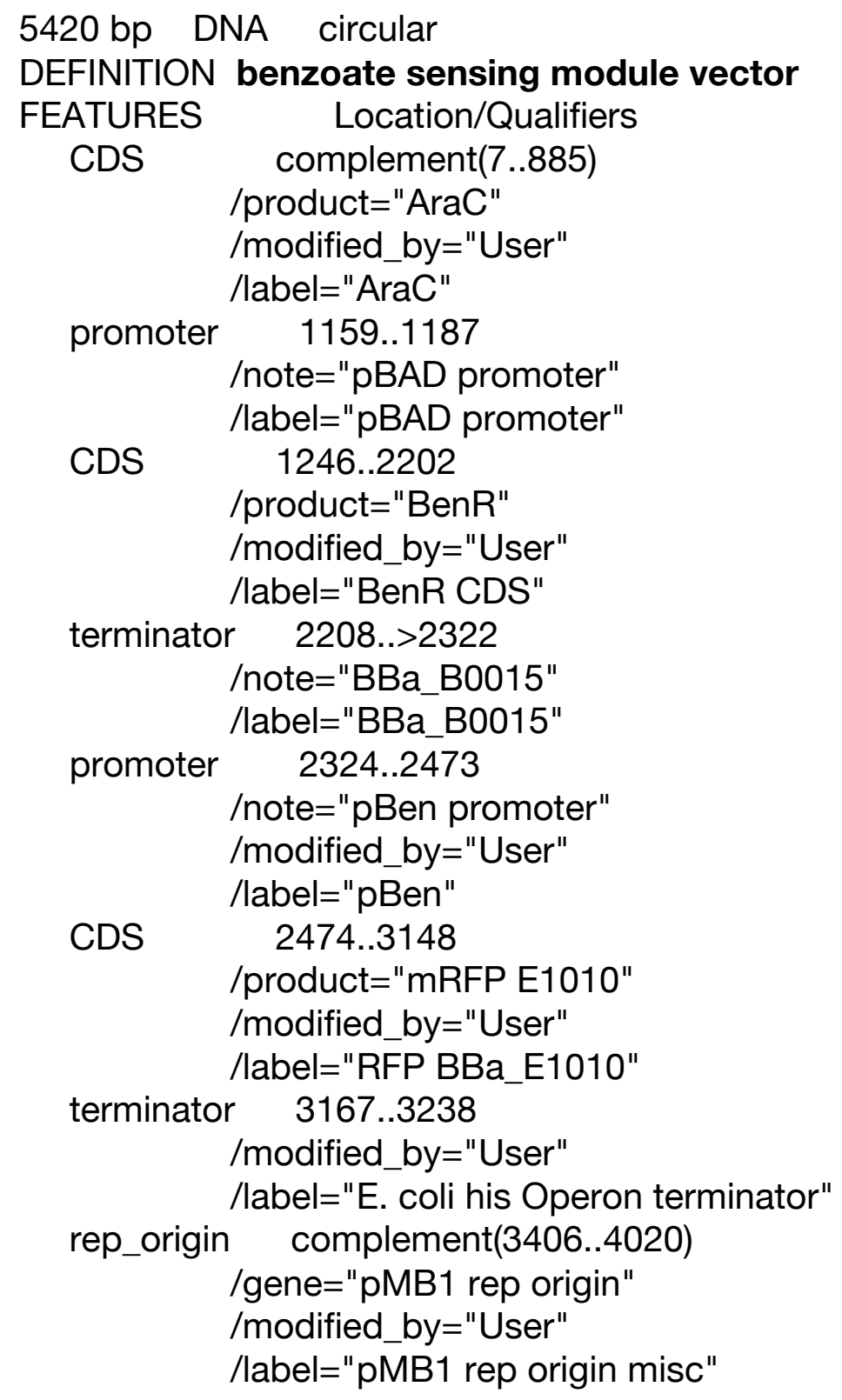


misc_feature complement(4240..5055)

/product="Kanamycin resistance marker CDS"

/modified_by="User"

/label="Kanamycin resistance marker CDS misc"

terminator $5301 . .5320$

/modified_by="User"

/label="Terminator"

ORIGIN

1 gacgtcttat gacaacttga cggctacatc attcactttt tcttcacaac cggcacggaa

61 ctcgctcggg ctggccccgg tgcattttt aaatacccgc gagaaataga gttgatcgtc

121 aaaaccaaca ttgcgaccga cggtggcgat aggcatccgg gtggtgctca aaagcagctt

181 cgcctggctg atacgttggt cctcgcgcca gcttaagacg ctaatcccta actgctggcg

241 gaaaagatgt gacagacgcg acggcgacaa gcaaacatgc tgtgcgacgc tggcgatatc

301 aaaattgctg tctgccaggt gatcgctgat gtactgacaa gcctcgcgta cccgattatc

361 catcggtgga tggagcgact cgttaatcgc ttccatgcgc cgcagtaaca attgctcaag

421 cagatttatc gccagcagct ccgaatagcg cccttcccct tgcccggcgt taatgatttg

481 cccaaacagg tcgctgaaat gcggctggtg cgcttcatcc gggcgaaaga accccgtatt

541 ggcaaatatt gacggccagt taagccattc atgccagtag gcgcgcggac gaaagtaaac

601 ccactggtga taccattcgc gagcctccgg atgacgaccg tagtgatgaa tctctcctgg

661 cgggaacagc aaaatatcac ccggtcggca aacaaattct cgtccctgat tttcaccac

721 ccctgaccg cgaatggtga gattgagaat ataaccttc attcccagcg gtcggtcgat

781 aaaaaaatcg agataaccgt tggcctcaat cggcgttaaa cccgccacca gatgggcatt

841 aaacgagtat cccggcagca ggggatcatt ttgcgcttca gccatacttt tcatactccc

901 gccattcaga gaagaaacca attgtccata ttgcatcaga cattgccgtc actgcgtctt

961 ttactggctc ttctcgctaa ccaaaccggt aaccccgctt attaaaagca ttctgtaaca

1021 aagcgggacc aaagccatga caaaaacgcg taacaaaagt gtctataatc acggcagaaa

1081 agtccacatt gattatttgc acggcgtcac actttgctat gccatagcat tttatccat

1141 aagattagcg gattctacct gacgctttt atcgcaactc tctactgttt ctccataccc

1201 gttttttgg gaattcaaaa gatcttttaa gaaggagata tacagatgga atctcgtctg

1261 ctgtctgaac gttcttctgt tttccaccac gctgacccgt acgctgtttc tgactacgtt

1321 aaccagcacg ttggtcagca ctgcatcggt ctgtctcgta ccacccaccc gcaggcttct

1381 ctgtctcacc gtaaattcgc tgaactggac ctgtgccgta tctcttacgg tggttctgtt

1441 cgtgttacct ctccggctct ggaaaccatc taccacctgc aggttctgct gaacggtaac

1501 tgcctgtggc gtggtcacaa acgtgaacag cacctggttc cgggtgaact gctgctgatc

1561 aacccggacg acccggttga cctgacctac tctgaagact gcgaaaaatt catcctgaaa

1621 gttccgaccc gtctgctgga ctctatctgc gacgaacagc gttggcagcg tccggacggt

1681 ggtgttcgtt tcctgcgtaa ccactaccgt ctggacgaac tggacggttt cgttaacctg

1741 ctggctatgg tttgccacga agctgaagtt tctgactctc tgccgcgtgt tcagggtcac

1801 tactctcaga tcgttgcttc taaactgctg accctgatgt ctaccaacat ccgtcgtgaa

1861 tctctgtctg ctccgcaggc tggtctggaa cgtatcctgg actacatcga acgtaacctg

1921 aaactggaac tgtctgctga agttctggct gaacaggctt gcatgtctct gcgttctctg

1981 tacgctctgt tcgaccagca cctgggtatc accccgaaac actacgttcg tcagcgtaaa

2041 ctggaacgtg ttcacgcttg cctgtctgac ccgacctgcg gtgttcgttc tgttaccgaa

2101 ctggctctgg actacggttt cctgcacctg ggtcgtttct ctgaaatcta ccgtcagcag

2161 ttcggtgaac tgccgtctca gaccttcaaa cgtcgtgctt aatagaccca ggcatcaaat

2221 aaaacgaaag gctcagtcga aagactgggc ctttcgtttt atctgttgtt tgtcggtgaa

2281 cgctctctac tagagtcaca ctggctcacc ttcgggtggg cctactgttc gaagcattgc

2341 cattttctga agttaccgaa aaagtaccga acatccgtaa atctggataa cgttctgcac 
2401 aatccggata gccccccgcc agccgtctcc ctaacctgac caggtctaaa caataacaag 2461 ggagagtctg gccatggctt cctccgaaga cgttatcaaa gagttcatgc gtttcaaagt 2521 tcgtatggaa ggttccgtta acggtcacga gttcgaaatc gaaggtgaag gtgaaggtcg 2581 tccgtacgaa ggtacccaga ccgctaaact gaaagttacc aaaggtggtc cgctgccgtt 2641 cgcttgggac atcctgtccc cgcagttcca gtacggttcc aaagcttacg ttaaacaccc 2701 ggctgacatc ccggactacc tgaaactgtc cttcccggaa ggtttcaaat gggaacgtgt 2761 tatgaacttc gaagacggtg gtgttgttac cgttacccag gactcctccc tgcaagacgg 2821 tgagttcatc tacaaagtta aactgcgtgg taccaacttc ccgtccgacg gtccggttat 2881 gcagaaaaaa accatgggtt gggaagcttc caccgaacgt atgtacccgg aagacggtgc 2941 tctgaaaggt gaaatcaaaa tgcgtctgaa actgaaagac ggtggtcact acgacgctga 3001 agttaaaacc acctacatgg ctaaaaaacc ggttcagctg ccgggtgctt acaaaaccga 3061 catcaaactg gacatcacct cccacaacga agactacacc atcgttgaac agtacgaacg 3121 tgctgaaggt cgtcactcca ccggtgctta atgcgtcttc ctgcagtccg gcaaaaaagg 3181 gcaaggtgtc accaccctgc ccttttctt taaaaccgaa aagattactt cgcgttatgc 3241 aggcttcctc gctcactgac tcgctgcgct cggtcgttcg gctgcggcga gcggtatcag 3301 ctcactcaaa ggcggtaata cggttatcca cagaatcagg ggataacgca ggaaagaaca 3361 tgtgagcaaa aggccagcaa aaggccagga accgtaaaaa ggccgcgttg ctggcgtttt 3421 tccacaggct ccgcccccct gacgagcatc acaaaaatcg acgctcaagt cagaggtggc 3481 gaaacccgac aggactataa agataccagg cgtttccccc tggaagctcc ctcgtgcgct 3541 ctcctgttcc gaccctgccg cttaccggat acctgtccgc ctttctccct tcgggaagcg 3601 tggcgcttc tcatagctca cgctgtaggt atctcagttc ggtgtaggtc gttcgctcca 3661 agctgggctg tgtgcacgaa ccccccgttc agcccgaccg ctgcgcctta tccggtaact 3721 atcgtcttga gtccaacccg gtaagacacg acttatcgcc actggcagca gccactggta 3781 acaggattag cagagcgagg tatgtaggcg gtgctacaga gttcttgaag tggtggccta 3841 actacggcta cactagaaga acagtatttg gtatctgcgc tctgctgaag ccagttacct 3901 tcggaaaaag agttggtagc tcttgatccg gcaaacaaac caccgctggt agcggtggtt $3961 \mathrm{ttttgtttg}$ caagcagcag attacgcgca gaaaaaaagg atctcaagaa gatcctttga 4021 tcttttctac ggggtctgac gctcagtgga acgaaaactc acgttaaggg attttggtca 4081 tgagattatc aaaaaggatc ttcacctaga tccttttaaa ttaaaaatga agttttaaat 4141 caatctaaag tatatatgag taaacttggt ctgacagctc gagtcccgtc aagtcagcgt 4201 aatgctctgc cagtgttaca accaattaac caattctgat tagaaaaact catcgagcat 4261 caaatgaaac tgcaatttat tcatatcagg attatcaata ccatatttt gaaaaagccg 4321 tttctgtaat gaaggagaaa actcaccgag gcagttccat aggatggcaa gatcctggta 4381 tcggtctgcg attccgactc gtccaacatc aatacaacct attaatttcc cctcgtcaaa 4441 aataaggtta tcaagtgaga aatcaccatg agtgacgact gaatccggtg agaatggcaa 4501 aagcttatgc atttctttcc agacttgttc aacaggccag ccattacgct cgtcatcaaa 4561 atcactcgca tcaaccaaac cgttattcat tcgtgattgc gcctgagcga gacgaaatac 4621 gcgatcgctg ttaaaaggac aattacaaac aggaatcgaa tgcaaccggc gcaggaacac 4681 tgccagcgca tcaacaatat ttcacctga atcaggatat tcttctaata cctggaatgc 4741 tgttttcccg gggatcgcag tggtgagtaa ccatgcatca tcaggagtac ggataaaatg 4801 cttgatggtc ggaagaggca taaattccgt cagccagttt agtctgacca tctcatctgt 4861 aacatcattg gcaacgctac ctttgccatg tttcagaaac aactctggcg catcgggctt 4921 cccatacaat cgatagattg tcgcacctga ttgcccgaca ttatcgcgag cccatttata 4981 cccatataaa tcagcatcca tgttggaatt taatcgcggc ctggagcaag acgtttcccg 5041 ttgaatatgg ctcataacac cccttgtatt actgtttatg taagcagaca gttttattgt 5101 tcatgatgat atatttttat cttgtgcaat gtaacatcag agattttgag acacaacgtg 5161 gctttgttga ataaatcgaa cttttgctga gttgaaggat cagctcgagt gccacctgac 5221 gtctaagaaa ccattattat catgacatta acctataaaa ataggcgtat cacgaggcag 
5281 aatttcagat aaaaaaaatc cttagctttc gctaaggatg atttctggaa ttcgaagagc 5341 ctcggtagtg atcttatttc attatggtga aagttggaac ctcttacgtg ccgatcaacg $/ /$

5401 tctcattttc gccagatatc

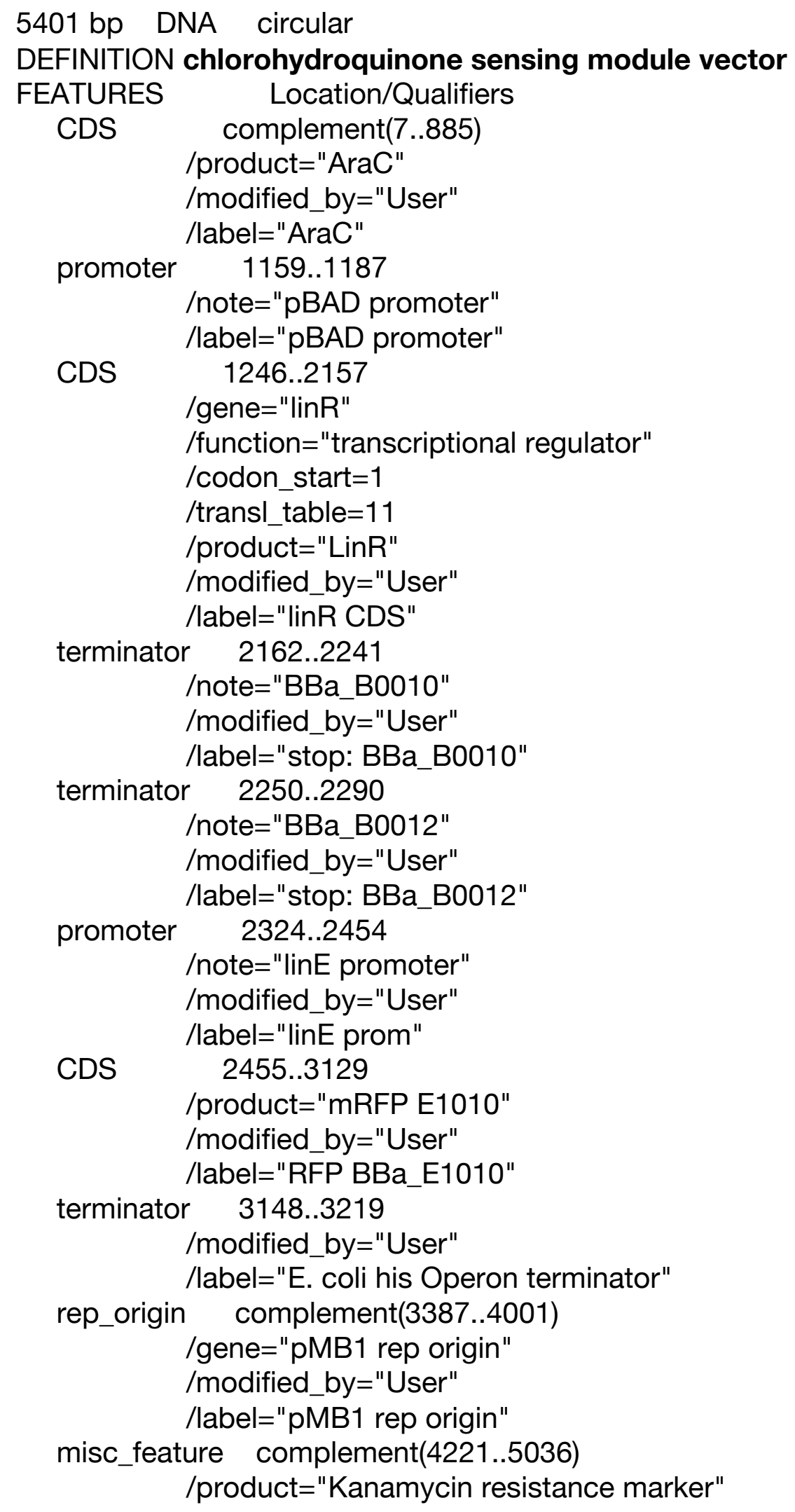




\author{
/modified_by="User" \\ /label="Kanamycin resistance marker " \\ stem_loop 5282..5301 \\ /label="Terminator" \\ ORIGIN
}

1 gacgtcttat gacaacttga cggctacatc attcactttt tcttcacaac cggcacggaa

61 ctcgctcggg ctggccccgg tgcattttt aaatacccgc gagaaataga gttgatcgtc

121 aaaaccaaca ttgcgaccga cggtggcgat aggcatccgg gtggtgctca aaagcagctt

181 cgcctggctg atacgttggt cctcgcgcca gcttaagacg ctaatcccta actgctggcg

241 gaaaagatgt gacagacgcg acggcgacaa gcaaacatgc tgtgcgacgc tggcgatatc

301 aaaattgctg tctgccaggt gatcgctgat gtactgacaa gcctcgcgta cccgattatc

361 catcggtgga tggagcgact cgttaatcgc ttccatgcgc cgcagtaaca attgctcaag

421 cagatttatc gccagcagct ccgaatagcg cccttcccct tgcccggcgt taatgatttg

481 cccaaacagg tcgctgaaat gcggctggtg cgcttcatcc gggcgaaaga accccgtatt

541 ggcaaatatt gacggccagt taagccattc atgccagtag gcgcgcggac gaaagtaaac

601 ccactggtga taccattcgc gagcctccgg atgacgaccg tagtgatgaa tctctcctgg

661 cgggaacagc aaaatatcac ccggtcggca aacaaattct cgtccctgat tttcaccac

721 cccctgaccg cgaatggtga gattgagaat ataacctttc attcccagcg gtcggtcgat

781 aaaaaaatcg agataaccgt tggcctcaat cggcgttaaa cccgccacca gatgggcatt

841 aaacgagtat cccggcagca ggggatcatt ttgcgettca gccatacttt tcatactccc

901 gccattcaga gaagaaacca attgtccata ttgcatcaga cattgccgtc actgcgtctt

961 ttactggctc ttctcgctaa ccaaaccggt aaccccgctt attaaaagca ttctgtaaca

1021 aagcgggacc aaagccatga caaaaacgcg taacaaaagt gtctataatc acggcagaaa

1081 agtccacatt gattatttgc acggcgtcac acttgctat gccatagcat tttatccat

1141 aagattagcg gattctacct gacgctttt atcgcaactc tctactgttt ctccataccc

1201 gttttttgg gaattcaaaa gatcttttaa gaaggagata tacagatgaa tatagatgac

1261 ctggatttc ggcacttggt gettttggat gcgctgttaa agcggcacag cgtcagcgcc

1321 gctgcacgag aactcgacct accgcagccc accgccagcc atgggctggc gcgcctgcgc

1381 aaagcattgg gcgacccgct tctcgtccgg gcgcgcgacg gcatggagcc gacaccacgt

1441 gccgaggcga tcgccggggt tgttcagcaa ctgctggaac tgcgtcgcga cctggctgaa

1501 ggcgggcaga cattctcacc tgaccgtctc aagcgcgaat tcatcattgc cggatcggac

1561 atcgcccatc ttgtcgtttt gacggcgttg cattcggcgg cgcggttcga ggcaccacac

1621 accagctacc gcgcactcac gctgagcggc gatgaaatgg tgagcgcgct cgagaccggg

1681 catgtcgata tcgccgtcgg cgcctatccg agccttgttg ccggcatcaa gacgcaacgg

1741 ctctaccagg aggaatatct gtgcttcgga aaggaaggtc atccgttcat caagtccggt

1801 gagaccgatg acttcacggc cgcagatcat atcgtggtca gcaccaaggg tatggcccat

1861 gcccatcgtg ccgtcgaacg cgctctcctc gacaagatcc atcccgatcg gatcaggatc

1921 gtcgcgagca gctttctcgt ggcgttggca gcttgcttcg agtcggacct tattcttacc

1981 gctccggctc gcgtgactgg tcggctcgcc gaggtctacg gactccgggc cgtgcggccg

2041 ccaattctta tggaagcatt tgaggtcagg caatattggc atgcgaggaa ccaagacgac

2101 ccgcctcacc gctggttgcg acagctcctg cacaaggtgc tgtccgcgcg gatgtgaaga

2161 cccaggcatc aaataaaacg aaaggctcag tcgaaagact gggcctttcg tttatctgt

2221 tgtttgtcgg tgaacgctct ctactagagt cacactgget caccttcggg tgggcctttc

2281 tgcgtttata tactagttgc gaatccaggt catctatatt cacaatctga attgtatcta

2341 tgaaggtccg ccaattgtca aatactccct gatccctcaa acaggctgca gcagcgatcg

2401 ttgtgcaggt ttgaagagac gatccgcaat cgcagaaaat agtggagagc gatcatggct

2461 tcctccgaag acgttatcaa agagttcatg cgtttcaaag ttcgtatgga aggttccgtt

2521 aacggtcacg agttcgaaat cgaaggtgaa ggtgaaggtc gtccgtacga aggtacccag 
2581 accgctaaac tgaaagttac caaaggtggt ccgctgccgt tcgcttggga catcctgtcc 2641 ccgcagttcc agtacggttc caaagcttac gttaaacacc cggctgacat cccggactac 2701 ctgaaactgt ccttcccgga aggtttcaaa tgggaacgtg ttatgaactt cgaagacggt 2761 ggtgttgtta ccgttaccca ggactcctcc ctgcaagacg gtgagttcat ctacaaagtt 2821 aaactgcgtg gtaccaactt cccgtccgac ggtccggtta tgcagaaaaa aaccatgggt 2881 tgggaagctt ccaccgaacg tatgtacccg gaagacggtg ctctgaaagg tgaaatcaaa 2941 atgcgtctga aactgaaaga cggtggtcac tacgacgctg aagttaaaac cacctacatg 3001 gctaaaaaac cggttcagct gccgggtgct tacaaaaccg acatcaaact ggacatcacc 3061 tcccacaacg aagactacac catcgttgaa cagtacgaac gtgctgaagg tcgtcactcc 3121 accggtgctt aatgcgtctt cctgcagtcc ggcaaaaaag ggcaaggtgt caccaccctg 3181 cccttttct ttaaaaccga aaagattact tcgcgttatg caggcttcct cgctcactga 3241 ctcgctgcgc tcggtcgttc ggctgcggcg agcggtatca gctcactcaa aggcggtaat 3301 acggttatcc acagaatcag gggataacgc aggaaagaac atgtgagcaa aaggccagca 3361 aaaggccagg aaccgtaaaa aggccgcgtt gctggcgttt ttccacaggc tccgcccccc 3421 tgacgagcat cacaaaaatc gacgctcaag tcagaggtgg cgaaacccga caggactata 3481 aagataccag gcgtttcccc ctggaagctc cctcgtgcge tctcctgttc cgaccctgcc 3541 gettaccgga tacctgtccg cctttctccc ttcgggaage gtggcgettt ctcatagctc 3601 acgctgtagg tatctcagtt cggtgtaggt cgttcgctcc aagctgggct gtgtgcacga 3661 accccccgtt cagcccgacc gctgcgcctt atccggtaac tatcgtcttg agtccaaccc 3721 ggtaagacac gacttatcgc cactggcagc agccactggt aacaggatta gcagagcgag 3781 gtatgtaggc ggtgctacag agttcttgaa gtggtggcct aactacggct acactagaag 3841 aacagtattt ggtatctgcg ctctgctgaa gccagttacc ttcggaaaaa gagttggtag 3901 ctcttgatcc ggcaaacaaa ccaccgctgg tagcggtggt tttttgttt gcaagcagca 3961 gattacgcgc agaaaaaaag gatctcaaga agatcctttg atcttttcta cggggtctga 4021 cgctcagtgg aacgaaaact cacgttaagg gattttggtc atgagattat caaaaaggat $4081 \mathrm{cttcacctag}$ atccttttaa attaaaaatg aagttttaaa tcaatctaaa gtatatatga 4141 gtaaacttgg tctgacagct cgagtcccgt caagtcagcg taatgctctg ccagtgttac 4201 aaccaattaa ccaattctga ttagaaaaac tcatcgagca tcaaatgaaa ctgcaattta 4261 ttcatatcag gattatcaat accatatttt tgaaaaagcc gtttctgtaa tgaaggagaa 4321 aactcaccga ggcagttcca taggatggca agatcctggt atcggtctgc gattccgact 4381 cgtccaacat caatacaacc tattaatttc ccctcgtcaa aaataaggtt atcaagtgag 4441 aaatcaccat gagtgacgac tgaatccggt gagaatggca aaagcttatg catttctttc 4501 cagacttgtt caacaggcca gccattacgc tcgtcatcaa aatcactcgc atcaaccaaa 4561 ccgttattca ttcgtgattg cgcctgagcg agacgaaata cgcgatcgct gttaaaagga 4621 caattacaaa caggaatcga atgcaaccgg cgcaggaaca ctgccagcgc atcaacaata $4681 \mathrm{ttttcacctg}$ aatcaggata ttcttctaat acctggaatg ctgttttccc ggggatcgca 4741 gtggtgagta accatgcatc atcaggagta cggataaaat gettgatggt cggaagaggc 4801 ataaattccg tcagccagtt tagtctgacc atctcatctg taacatcatt ggcaacgcta 4861 cctttgccat gtttcagaaa caactctggc gcatcggget tcccatacaa tcgatagatt 4921 gtcgcacctg attgcccgac attatcgcga gcccatttat acccatataa atcagcatcc 4981 atgttggaat ttaatcgcgg cctggagcaa gacgtttccc gttgaatatg gctcataaca 5041 ccccttgtat tactgtttat gtaagcagac agttttattg ttcatgatga tatatttta 5101 tcttgtgcaa tgtaacatca gagattttga gacacaacgt ggctttgttg aataaatcga 5161 acttttgctg agttgaagga tcagctcgag tgccacctga cgtctaagaa accattatta 5221 tcatgacatt aacctataaa aataggcgta tcacgaggca gaatttcaga taaaaaaaat 5281 ccttagcttt cgctaaggat gatttctgga attcgaagag cctcggtagt gatcttattt 5341 cattatggtg aaagttggaa cctcttacgt gccgatcaac gtctcatttt cgccagatat $5401 \mathrm{c}$ 
7143 bp DNA circular

DEFINITION CocE metabolic module vector

FEATURES Location/Qualifiers

CDS complement(155..>1246)

/product="Lacl"

/transl_table $=11$

/modified_by="User"

/label="lacl"

promoter 1624..1642

/note="T7 promoter"

/modified_by="User"

/label="T7 prom"

misc_feature 1642..1669

/note="LacO"

/modified_by="User"

/label="lacO "

CDS 1711..3435

/product $=$ "CocE"

/label="CocE"

terminator 3658.3687

/modified_by="User"

/label="Terminator"

CDS 4498..5448

/product="RepA"

/modified_by="User"

/label="repA CDS"

misc_feature $5747 . .6937$

/product="tetracycline resistance marker"

/modified_by="User"

ORIGIN

/label="tetR CDS"

1 aattcgaaga cgtcagaaga cagtcataag tgcggcgacg atagtcatgc cccgcgccca

61 ccggaaggag ctgactgggt tgaaggctct caagggcatc ggtcgagatc ccggtgccta 121 atgagtgagc taacttacat taattgcgtt gcgctcactg cccgctttcc agtcgggaaa

181 cctgtcgtgc cagctgcatt aatgaatcgg ccaacgcgcg gggagaggcg gtttgcgtat 241 tgggcgccag ggtggttttt cttttcacca gtgagacggg caacagctga ttgcccttca 301 ccgcctggcc ctgagagagt tgcagcaagc ggtccacgct ggtttgcccc agcaggcgaa 361 aatcctgtt gatggtggtt aacggcggga tataacatga gctgtcttcg gtatcgtcgt 421 atcccactac cgagatatcc gcaccaacgc gcagcccgga ctcggtaatg gcgcgcattg 481 cgcccagcgc catctgatcg ttggcaacca gcatcgcagt gggaacgatg ccctcattca 541 gcatttgcat ggtttgttga aaaccggaca tggcactcca gtcgccttcc cgttccgcta 601 tcggctgaat ttgattgcga gtgagatatt tatgccagcc agccagacgc agacgcgccg 661 agacagaact taatgggecc gctaacagcg cgatttgctg gtgacccaat gcgaccagat 
721 gctccacgcc cagtcgcgta ccgtcttcat gggagaaaat aatactgttg atgggtgtct 781 ggtcagagac atcaagaaat aacgccggaa cattagtgca ggcagcttcc acagcaatgg 841 catcctggtc atccagcgga tagttaatga tcagcccact gacgcgttgc gcgagaagat 901 tgtgcaccgc cgctttacag gcttcgacgc cgcttcgttc taccatcgac accaccacgc 961 tggcacccag ttgatcggcg cgagatttaa tcgccgcgac aatttgcgac ggcgcgtgca 1021 gggccagact ggaggtggca acgccaatca gcaacgactg tttgcccgcc agttgttgtg 1081 ccacgcggtt gggaatgtaa ttcagctccg ccatcgccgc ttccactttt tcccgcgttt 1141 tcgcagaaac gtggctggcc tggttcacca cgcgggaaac ggtctgataa gagacaccgg 1201 catactctgc gacatcgtat aacgttactg gtttcacatt caccaccctg aattgactct 1261 cttccgggcg ctatcatgcc ataccgcgaa aggttttgcg ccattcgatg gtgtccggga 1321 tctcgacgct ctccettatg cgactcctgc attaggaagc agcccagtag taggttgagg 1381 ccgttgagca ccgccgccgc aaggaatggt gcatgcaagg agatggcgcc caacagtccc 1441 ccggccacgg ggcctgccac catacccacg ccgaaacaag cgctcatgag cccgaagtgg 1501 cgagcccgat cttccccatc ggtgatgtcg gcgatatagg cgccagcaac cgcacctgtg 1561 gcgccggtga tgccggccac gatgcgtccg gcgtagagga tcgagatctc gatcccgcga 1621 aattaatacg actcactata ggggaattgt gagcggataa caattcccct gtagaaataa $1681 \mathrm{tttg} t t+a a$ ctttaagaag gagatatacc atggttgacg gtaactactc tgttgcttct 1741 aacgttatgg ttccgatgcg tgacggtgtt cgtctggctg ttgacctgta ccgtccggac 1801 gctgacggtc cggttccggt tctgctggtt cgtaacccgt acgacaaatt cgacgttttc 1861 gcttggtcta cccagtctac caactggctg gaatttgttc gtgacggtta cgctgttgtt 1921 atccaggaca cccgtggtct gttcgcttct gaaggtgaat ttgttccgca cgttgacgac 1981 gaagctgacg ctgaagacac cctgtcttgg atcctggaac aggcttggtg cgacggtaac 2041 gttggtatgt tcggtgtttc ttacctgggt gttacccagt ggcaggctgc tgtttctggt 2101 gttggtggtc tgaaagctat cgctccgtct atggettctg ctgacctgta ccgtgctccg 2161 tggtacggtc cgggtggtgc tctgtctgtt gaagctctgc tgggttggtc tgctctgatc 2221 ggtaccggtc tgatcacctc tcgttctgac gctcgtccgg aagacgctgc tgacttcgtt 2281 cagctggctg ctatcctgaa cgacgttgct ggtgctgctt ctgttacccc gctggctgaa 2341 cagccgctgc tgggtcgtct gatcccgtgg gttatcgacc aggttgttga ccacccggac 2401 aacgacgaat cttggcagtc tatctctctg ttcgaacgtc tgggtggtct ggctaccccg 2461 gctctgatca ccgctggttg gtacgacggt ttcgttggtg aatctctgcg taccttcgtt 2521 gctgttaaag acaacgctga cgctcgtctg gttgttggtc cgtggtccca ctctaacctg 2581 accggtcgta acgctgaccg taaattcggt atcgctgcta cctacccgat ccaggaagct 2641 accaccatgc acaaagcttt cttcgaccgt cacctgcgtg gtgaaaccga cgctctggct 2701 ggtgttccga aagttcgtct gttcgttatg ggtatcgacg aatggcgtga cgaaaccgac 2761 tggccgctgc cggacaccgc ttacaccccg ttctacctgg gtggttctgg tgctgctaac 2821 acctctaccg gtggtggtac cctgtctacc tctatctctg gtaccgaatc tgctgacacc 2881 tacctgtacg acccggctga cccggttccg tctctgggtg gtaccctgct gttccacaac 2941 ggtgacaacg gtccggctga ccagcgtccg atccacgacc gtgacgacgt tctgtgctac 3001 tctaccgaag ttctgaccga cccggttgaa gttaccggta ccgtttctgc tcgtctgttc 3061 gtttcttctt ctgctgttga caccgacttc accgctaaac tggttgacgt tttcccggac 3121 ggtcgtgcta tcgctctgtg cgacggtatc gttcgtatgc gttaccgtga aaccctggtt 3181 aacccgaccc tgatcgaagc tggtgaaatc tacgaagttg ctatcgacat gctggctacc 3241 tctaacgttt tcctgccggg tcaccgtatc atggttcagg tttcttcttc taacttcccg 3301 aaatacgacc gtaactctaa caccggtggt gttatcgctc gtgaacagct ggaagaaatg 3361 tgcaccgctg ttaaccgtat ccaccgtggt ccggaacacc cgtctcacat cgttctgccg 3421 atcatcaaac gttaaagcgt cttcctgcag gagtcactaa gggttagtta gttagattag 3481 cagaaagtca aaagcctccg accggagget tttgactaaa acttcccttg gggttatcat 3541 tggggctcac tcaaaggcgg taatcagata aaaaaaatcc ttagctttcg ctaaggatga 
3601 tttctgctag agctgtcaga ccaagtttac gagctcgctt ggactcctgt tgatagatcc 3661 agtaatgacc tcagaactcc atctggattt gttcagaacg ctcggttgcc gccgggcgtt $3721 \mathrm{ttttattggt} \mathrm{gagaatccaa}$ gcactaggga cagtaagacg ggtaagcctg ttgatgatac 3781 cgctgcctta ctgggtgcat tagccagtct gaatgacctg tcacgggata atccgaagtg 3841 gtcagactgg aaaatcagag ggcaggaact gctgaacagc aaaaagtcag atagcaccac 3901 atagcagacc cgccataaaa cgccctgaga agcccgtgac gggcttttct tgtattatgg 3961 gtagtttcct tgcatgaatc cataaaagge gectgtagtg ccatttaccc ccattcactg 4021 ccagagccgt gagcgcagcg aactgaatgt cacgaaaaag acagcgactc aggtgcctga 4081 tggtcggaga caaaaggaat attcagcgat ttgcccgagc ttgcgagggt gctacttaag 4141 cctttagggt tttaaggtct gttttgtaga ggagcaaaca gcgtttgcga catccttttg 4201 taatactgcg gaactgacta aagtagtgag ttatacacag ggctgggatc tattcttttt 4261 atctttttt attctttctt tattctataa attataacca cttgaatata aacaaaaaa 4321 acacacaaag gtctagcgga atttacagag ggtctagcag aatttacaag ttttccagca 4381 aaggtctagc agaatttaca gatacccaca actcaaagga aaaggacatg taattatcat 4441 tgactagccc atctcaattg gtatagtgat taaaatcacc tagaccaatt gagatgtatg 4501 tctgaattag ttgttttcaa agcaaatgaa ctagcgatta gtcgctatga cttaacggag 4561 catgaaacca agctaatttt atgctgtgtg gcactactca accccacgat tgaaaaccct 4621 acaaggaaag aacggacggt atcgttcact tataaccaat acgctcagat gatgaacatc 4681 agtagggaaa atgcttatgg tgtattagct aaagcaacca gagagctgat gacgagaact 4741 gtggaaatca ggaatccttt ggttaaaggc tttgagattt tccagtggac aaactatgcc 4801 aagttctcaa gcgaaaaatt agaattagtt tttagtgaag agatattgcc ttatcttttc 4861 cagttaaaaa aattcataaa atataatctg gaacatgtta agtctttga aaacaaatac 4921 tctatgagga tttatgagtg gttattaaaa gaactaacac aaaagaaaac tcacaaggca 4981 aatatagaga ttagccttga tgaatttaag ttcatgttaa tgcttgaaaa taactaccat 5041 gagtttaaaa ggcttaacca atgggttttg aaaccaataa gtaaagattt aaacacttac 5101 agcaatatga aattggtggt tgataagcga ggccgcccga ctgatacgtt gattttccaa 5161 gttgaactag atagacaaat ggatctcgta accgaacttg agaacaacca gataaaaatg 5221 aatggtgaca aaataccaac aaccattaca tcagattcct acctacgtaa cggactaaga 5281 aaaacactac acgatgcttt aactgcaaaa attcagctca ccagttttga ggcaaaattt 5341 ttgagtgaca tgcaaagtaa gcatgatctc aatggttcgt tctcatggct cacgcaaaaa 5401 caacgaacca cactagagaa catactggct aaatacggaa ggatctgagg ttcttatggc $5461 \mathrm{tcttg} t a t c t$ atcagtgaag catcaagact aacaaacaaa agtagaacaa ctgttcaccg 5521 ttagatatca aagggaaaac tgtccatatg cacagatgaa aacggtgtaa aaaagataga 5581 tacatcagag cttttacgag ttttggtgc atttaaagct gttcaccatg aacagatcga 5641 caatgtaact actagaggag attctcatgt ttgacagctt atcatcgata agctttaatg 5701 cggtagttta tcacagttaa attgctaacg cagtcaggca ccgtgtatga aatctaacaa 5761 tgcgctcatc gtcattctcg gcaccgtcac cctggacgct gtaggcatag gcttggttat 5821 gccggtactg ccgggcctct tgcgggatat cgtccattcc gacagtattg ccagtcacta 5881 tggcgtgctg cttgcgctct atgcgttgat gcaatttctt tgcgcacccg ttctcggagc 5941 cctgtccgac cgctttggcc gccgtccagt cctgctcgct tcgctccttg gagccactat 6001 cgactacgcg atcatggcga ccacacccgt cctgtggatt ctctacgccg gacgcatcgt 6061 ggcgggcatc acgggtgcca caggtgcggt tgctggtgcc tatatcgccg acatcaccga 6121 tggggaagat cgggctcgcc acttcgggct catgagcgct tgtttcggcg tgggtatggt 6181 ggcaggeccc gtggccgggg gactgttggg tgccatctcc ttgcatgcac cattccttgc 6241 ggcggcggtg ctcaacggcc tcaacctcct cctgggctgc ttccttatgc aggaatcgca 6301 taagggagag cgccgtccga tgcccttgcg tgccttcaat ccagtcagct ccttccggtg 6361 ggcgcggggc atgactatcg tcgccgcact tatgactgtt ttctttatca tgcaactcgt 6421 aggacaggtt ccggcagcgc tctgggtcat tttcggcgag gaccgctttc gctggagcgc 
6481 gacgatgatc ggcctgtcgc ttgcggtatt cggaatcttg cacgccctcg ctcaagcctt 6541 cgtcacggge cccgccacca aacgtttcgg cgagaagcag gccattatcg cgggcatggc 6601 ggccgacgcg ctgggctacg tcttgctggc gttcgcgacg cgcggctgga tggecttccc 6661 cattatgatt cttctcgctt ccggcggcat cggtatgccc gcgttgcagg ccatgctgtc 6721 ccgccaagta gatgacgacc atcagggaca gcttcaaggg tcgctcgcgg ctcttaccag 6781 cctcacttcg atcattggac cgctgatcgt cacggcgatt tatgccgcct cggcgagcac 6841 atggaacggg ttggcatgga ttgtaggtgc cgccctttac cttgtctgcc tccccgcgtt 6901 gcgtcgcggt gcatggagcc gggccacctc gacctaataa tactagctcc ggcaaaaaaa 6961 cgggcaaggt gtcaccaccc tgcccttttt ctttaaaacc gaaaagatta cttcgcgttt 7021 gccacctgac gtctaagaaa aggaatattc agcaatttgc ccgtgccgaa gaaaggccca 7081 cccgtgaagg tgagccagtg agttgattgc tacgtaatta gttagttagc ccttagtgac // $7141 \mathrm{tcg}$

6570 bp DNA circular DEFINITION HipO metabolic module vector FEATURES Location/Qualifiers CDS complement(155..>1246) $/$ product $=$ "Lacl" /modified_by="User" /label="lacl"

promoter 1624..1642 $/$ note=" promoter T7" /modified_by="User" /label="T7 prom"

misc_feature 1642..1669 /note="LacO" /modified_by="User" /label="lacO reg"

CDS 1711..2862 /product="HipO" /modified_by="User" /label="hipO"

terminator 3085.3114 /modified_by="User" /label="Terminator"

CDS 3925..4875 /product="RepA" /modified_by="User" /label="repA"

misc_feature $5174 . .6364$ /product="tetracycline resistance marker" /modified_by="User"

ORIGIN /label="tetR CDS" 
1 aattcgaaga cgtcagaaga cagtcataag tgcggcgacg atagtcatgc cccgcgccca

61 ccggaaggag ctgactgggt tgaaggctct caagggcatc ggtcgagatc ccggtgccta 121 atgagtgagc taacttacat taattgcgtt gcgctcactg cccgctttcc agtcgggaaa 181 cctgtcgtgc cagctgcatt aatgaatcgg ccaacgcgcg gggagaggcg gtttgcgtat 241 tgggcgccag ggtggttttt cttttcacca gtgagacggg caacagctga ttgcccttca 301 ccgcctggcc ctgagagagt tgcagcaagc ggtccacgct ggtttgcccc agcaggcgaa 361 aatcctgttt gatggtggtt aacggcggga tataacatga gctgtcttcg gtatcgtcgt 421 atcccactac cgagatatcc gcaccaacgc gcagcccgga ctcggtaatg gcgcgcattg 481 cgcccagcgc catctgatcg ttggcaacca gcatcgcagt gggaacgatg ccctcattca 541 gcatttgcat ggtttgttga aaaccggaca tggcactcca gtcgccttcc cgttccgcta 601 tcggctgaat ttgattgcga gtgagatatt tatgccagcc agccagacgc agacgcgccg 661 agacagaact taatgggccc gctaacagcg cgatttgctg gtgacccaat gcgaccagat 721 gctccacgcc cagtcgcgta ccgtcttcat gggagaaaat aatactgttg atgggtgtct 781 ggtcagagac atcaagaaat aacgccggaa cattagtgca ggcagcttcc acagcaatgg 841 catcctggtc atccagcgga tagttaatga tcagcccact gacgcgttgc gcgagaagat 901 tgtgcaccgc cgctttacag gcttcgacgc cgcttcgttc taccatcgac accaccacgc 961 tggcacccag ttgatcggcg cgagatttaa tcgccgcgac aatttgcgac ggcgcgtgca 1021 gggccagact ggaggtggca acgccaatca gcaacgactg tttgcccgcc agttgttgtg 1081 ccacgcggtt gggaatgtaa ttcagctccg ccatcgccgc ttccactttt tcccgcgttt 1141 tcgcagaaac gtggctggcc tggttcacca cgcgggaaac ggtctgataa gagacaccgg 1201 catactctgc gacatcgtat aacgttactg gtttcacatt caccaccctg aattgactct 1261 cttccgggcg ctatcatgcc ataccgcgaa aggttttgcg ccattcgatg gtgtccggga 1321 tctcgacgct ctccettatg cgactcctgc attaggaagc agcccagtag taggttgagg 1381 ccgttgagca ccgccgccgc aaggaatggt gcatgcaagg agatggcgcc caacagtccc 1441 ccggccacgg ggcctgccac catacccacg ccgaaacaag cgctcatgag cccgaagtgg 1501 cgagcccgat cttccccatc ggtgatgtcg gcgatatagg cgccagcaac cgcacctgtg 1561 gcgccggtga tgccggccac gatgcgtccg gcgtagagga tcgagatctc gatcccgcga 1621 aattaatacg actcactata ggggaattgt gagcggataa caattcccct gtagaaataa $1681 \mathrm{ttttgtttaa}$ ctttaagaag gagatatacc atgaacctga tcccggaaat cctggacctg 1741 cagggtgaat tcgaaaaaat ccgtcaccag atccacgaaa acccggaact gggtttcgac 1801 gaactgtgca ccgctaaact ggttgctcag aaactgaaag aattcggtta cgaagtttac 1861 gaagaaatcg gtaaaaccgg tgttgttggt gttctgaaaa aaggtaactc tgacaaaaaa 1921 atcggtctgc gtgctgacat ggacgctctg ccgctgcagg aatgcaccaa cctgccgtac 1981 aaatctaaaa aagaaaacgt tatgcacgct tgcggtcacg acggtcacac cacctctctg 2041 ctgctggctg ctaaatacct ggcttctcag aacttcaacg gtgctctgaa cctgtacttc 2101 cagccggctg aagaaggtct gggtggtgct aaagctatga tcgaagacgg tctgttcgaa 2161 aaattcgact ctgactacgt tttcggttgg cacaacatgc cgttcggttc tgacaaaaaa 2221 ttctacctga aaaaaggtgc tatgatgget tcttctgact cttactctat cgaagttatc 2281 ggtcgtggtg gtcacggttc tgctccggaa aaagctaaag acccgatcta cgctgcttct 2341 ctgctgatcg ttgctctgca gtctatcgtt tctcgtaacg ttgacccgca gaactctgct 2401 gttgtttcta tcggtgcttt caacgctggt cacgctttca acatcatccc ggacatcgct 2461 accatcaaaa tgtctgttcg tgctctggac aacgaaaccc gtaaactgac cgaagaaaaa 2521 atctacaaaa tctgcaaagg tatcgctcag gctaacgaca tcgaaatcaa aatcaacaaa 2581 aacgttgttg ctccggttac catgaacaac gacgaagctg ttgacttcgc ttctgaagtt 2641 gctaaagaac tgttcggtga aaaaaactgc gaattcaacc accgtccgct gatggcttct 2701 gaagacttcg gtttcttctg cgaaatgaaa aaatgcgctt acgctttcct ggaaaacgaa 2761 aacgacatct acctgcacaa ctcttcttac gttttcaacg acaaactgct ggctcgtgct 2821 gcttcttact acgctaaact ggctctgaaa tacctgaaat aaagcgtctt cctgcaggag 
2881 tcactaaggg ttagttagtt agattagcag aaagtcaaaa gcctccgacc ggaggctttt 2941 gactaaaact tcccttgggg ttatcattgg ggctcactca aaggcggtaa tcagataaaa 3001 aaaatcctta gctttcgcta aggatgattt ctgctagagc tgtcagacca agtttacgag 3061 ctcgcttgga ctcctgttga tagatccagt aatgacctca gaactccatc tggatttgtt 3121 cagaacgctc ggttgccgcc gggcgtttt tattggtgag aatccaagca ctagggacag 3181 taagacgggt aagcctgttg atgataccgc tgccttactg ggtgcattag ccagtctgaa 3241 tgacctgtca cgggataatc cgaagtggtc agactggaaa atcagagggc aggaactgct 3301 gaacagcaaa aagtcagata gcaccacata gcagacccgc cataaaacgc cctgagaagc 3361 ccgtgacggg cttttcttgt attatgggta gtttccttgc atgaatccat aaaaggcgcc 3421 tgtagtgcca tttaccccca ttcactgcca gagccgtgag cgcagcgaac tgaatgtcac 3481 gaaaaagaca gcgactcagg tgcctgatgg tcggagacaa aaggaatatt cagcgatttg 3541 cccgagcttg cgagggtgct acttaagcct ttagggtttt aaggtctgtt ttgtagagga 3601 gcaaacagcg tttgcgacat ccttttgtaa tactgcggaa ctgactaaag tagtgagtta 3661 tacacagggc tgggatctat tctttttatc ttttttatt ctttctttat tctataaatt 3721 ataaccactt gaatataaac aaaaaaaca cacaaaggtc tagcggaatt tacagagggt 3781 ctagcagaat ttacaagttt tccagcaaag gtctagcaga atttacagat acccacaact 3841 caaaggaaaa ggacatgtaa ttatcattga ctagcccatc tcaattggta tagtgattaa 3901 aatcacctag accaattgag atgtatgtct gaattagttg ttttcaaagc aaatgaacta 3961 gcgattagtc gctatgactt aacggagcat gaaaccaagc taattttatg ctgtgtggca 4021 ctactcaacc ccacgattga aaaccctaca aggaaagaac ggacggtatc gttcacttat 4081 aaccaatacg ctcagatgat gaacatcagt agggaaaatg cttatggtgt attagctaaa 4141 gcaaccagag agctgatgac gagaactgtg gaaatcagga atcctttggt taaaggcttt 4201 gagattttcc agtggacaaa ctatgccaag ttctcaagcg aaaaattaga attagttttt 4261 agtgaagaga tattgcctta tctttccag ttaaaaaaat tcataaaata taatctggaa 4321 catgttaagt cttttgaaaa caaatactct atgaggattt atgagtggtt attaaaagaa 4381 ctaacacaaa agaaaactca caaggcaaat atagagatta gccttgatga atttaagttc 4441 atgttaatgc ttgaaaataa ctaccatgag tttaaaaggc ttaaccaatg ggttttgaaa 4501 ccaataagta aagatttaaa cacttacagc aatatgaaat tggtggttga taagcgaggc 4561 cgcccgactg atacgttgat tttccaagtt gaactagata gacaaatgga tctcgtaacc 4621 gaacttgaga acaaccagat aaaaatgaat ggtgacaaaa taccaacaac cattacatca 4681 gattcctacc tacgtaacgg actaagaaaa acactacacg atgctttaac tgcaaaaatt 4741 cagctcacca gttttgaggc aaaatttttg agtgacatgc aaagtaagca tgatctcaat 4801 ggttcgttct catggctcac gcaaaaacaa cgaaccacac tagagaacat actggctaaa 4861 tacggaagga tctgaggttc ttatggctct tgtatctatc agtgaagcat caagactaac 4921 aaacaaaagt agaacaactg ttcaccgtta gatatcaaag ggaaaactgt ccatatgcac 4981 agatgaaaac ggtgtaaaaa agatagatac atcagagctt ttacgagttt ttggtgcatt 5041 taaagctgtt caccatgaac agatcgacaa tgtaactact agaggagatt ctcatgtttg 5101 acagcttatc atcgataagc tttaatgcgg tagtttatca cagttaaatt gctaacgcag 5161 tcaggcaccg tgtatgaaat ctaacaatgc gctcatcgtc attctcggca ccgtcaccct 5221 ggacgctgta ggcatagget tggttatgcc ggtactgccg ggcctcttgc gggatatcgt 5281 ccattccgac agtattgcca gtcactatgg cgtgctgctt gcgctctatg cgttgatgca 5341 atttctttgc gcacccgttc tcggagccct gtccgaccgc tttggccgcc gtccagtcct 5401 gctcgcttcg ctccttggag ccactatcga ctacgcgatc atggcgacca cacccgtcct 5461 gtggattctc tacgccggac gcatcgtggc gggcatcacg ggtgccacag gtgcggttgc 5521 tggtgcctat atcgccgaca tcaccgatgg ggaagatcgg gctcgccact tcgggctcat 5581 gagcgettgt ttcggcgtgg gtatggtggc aggccccgtg gccgggggac tgttgggtgc 5641 catctccttg catgcaccat tccttgcggc ggcggtgctc aacggcctca acctcctcct 5701 gggctgcttc cttatgcagg aatcgcataa gggagagcge cgtccgatgc ccttgcgtgc 
5761 cttcaatcca gtcagctcct tccggtgggc gcggggcatg actatcgtcg ccgcacttat 5821 gactgttttc tttatcatgc aactcgtagg acaggttccg gcagcgctct gggtcatttt 5881 cggcgaggac cgctttcgct ggagcgcgac gatgatcgge ctgtcgcttg cggtattcgg 5941 aatcttgcac gecctcgctc aagccttcgt cacgggeccc gccaccaaac gtttcggcga 6001 gaagcaggcc attatcgcgg gcatggcggc cgacgcgctg ggctacgtct tgctggcgtt 6061 cgcgacgcgc ggctggatgg ccttccccat tatgattctt ctcgettccg gcggcatcgg 6121 tatgcccgcg ttgcaggcca tgctgtcccg ccaagtagat gacgaccatc agggacagct 6181 tcaagggtcg ctcgcggctc ttaccagcct cacttcgatc attggaccgc tgatcgtcac 6241 ggcgatttat gccgcctcgg cgagcacatg gaacgggttg gcatggattg taggtgccgc 6301 cctttacctt gtctgcctcc ccgcgttgcg tcgcggtgca tggagccggg ccacctcgac 6361 ctaataatac tagctccggc aaaaaaacgg gcaaggtgtc accaccctgc ccttttctt 6421 taaaaccgaa aagattactt cgcgtttgcc acctgacgtc taagaaaagg aatattcagc 6481 aatttgcccg tgccgaagaa aggcccaccc gtgaaggtga gccagtgagt tgattgctac // 6541 gtaattagtt agttagccct tagtgactcg

$3338 \mathrm{bp}$ DNA circular DEFINITION NemA metabolic module vector

FEATURES Location/Qualifiers

promoter $5 . .44$

/note="J23100 promoter"

/modified_by="User"

/label="j23100"

CDS $\quad 67 . .1164$

/gene="nemA"

/locus_tag="b1650"

/gene_synonym="ECK1646; JW1642; ydhN"

/EC_number="1.-.-.-"

/function="enzyme; Central intermediary metabolism: Pool, multipurpose conversions"

/GO_process="GO:0006805 - xenobiotic metabolic process" /note $=$ "N-ethylmaleimide reductase"

/codon_start $=1$

/transl_table $=11$

/product="N-ethylmaleimide reductase, FMN-linked"

/protein_id="NP_416167.1"

/db_xref="Gl:16129608"

/db_xref="ASAP:ABE-0005516"

/db_xref="UniProtKB/Swiss-Prot:P77258"

/db_xref="EcoGene:EG13546"

/db_xref="GenelD:946164"

/translation="MSSEKLYSPLKVGAITAANRIFMAPLTRLRSIEPGDIPTPLMAE

YYRQRASAGLIISEATQISAQAKGYAGAPGIHSPEQIAAWKKITAGVHAENGHMAVQL 
WHTGRISHASLQPGGQAPVAPSALSAGTRTSLRDENGQAIRVETSMPRALELEEIPGI

VNDFRQAIANAREAGFDLVELHSAHGYLLHQFLSPSSNHRTDQYGGSVENRARLVLEV

VDAGIEEWGADRIGIRVSPIGTFQNTDNGPNEEADALYLIEQLGKRGIAYLHMSEPDW

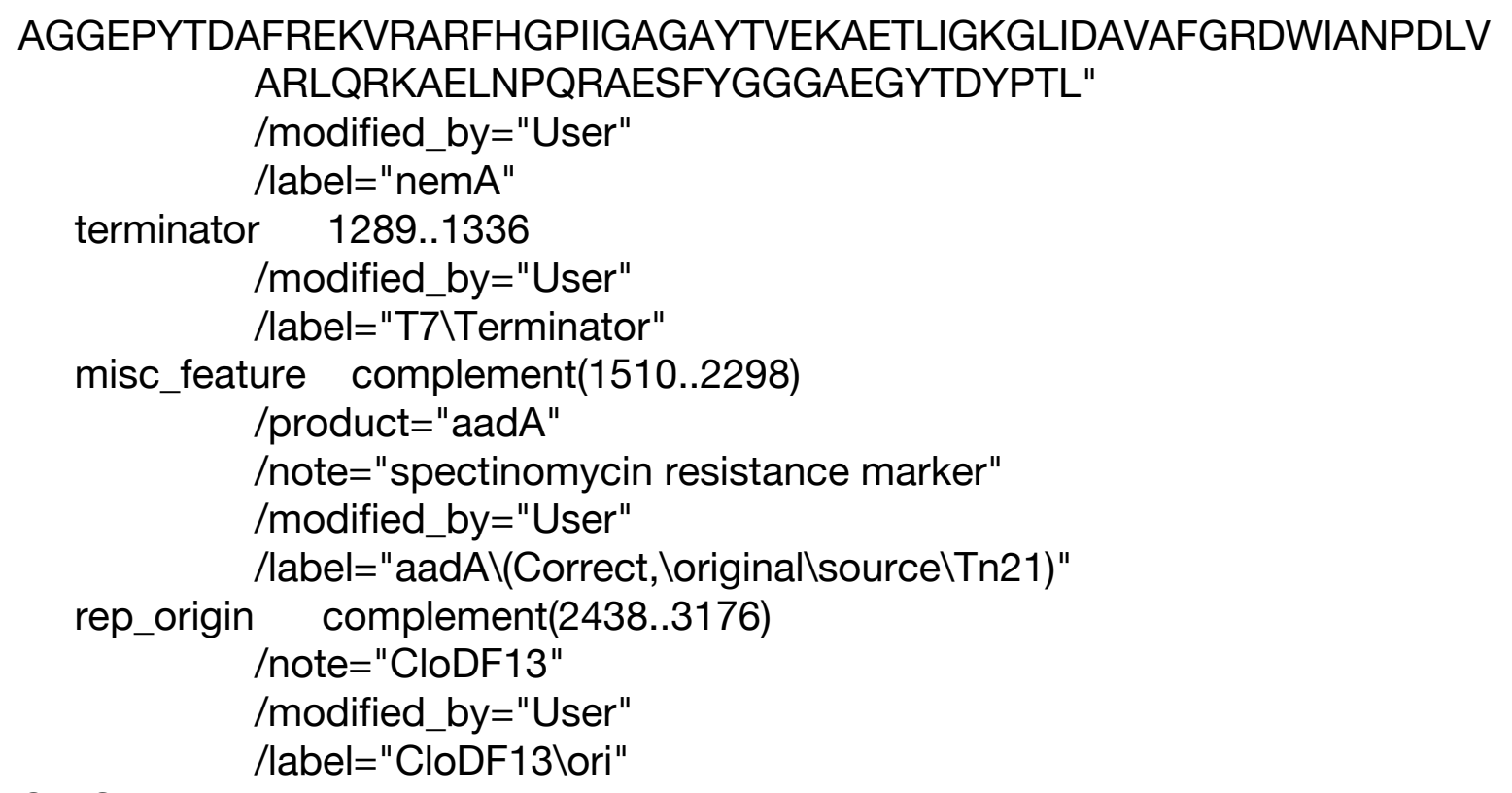

ORIGIN

1 cgtcgccttt gacggctagc tcagtcctag gtacagtgct agcccaccag acgaccggga

61 gcctttatgt catctgaaaa actgtattcc ccactgaaag tgggcgcgat cacggcggca

121 aaccgtattt ttatggcacc gctgacgcgt ctgcgcagta ttgaaccggg tgacattcct

181 acccogttga tggcggaata ctatcgccaa cgtgccagtg ccggtttgat tattagtgaa

241 gccacgcaaa tttctgccca ggcaaaagga tatgcaggtg cgcctggcat ccatagtccg

301 gagcaaattg ccgcatggaa aaaaatcacc gctggcgttc atgctgaaaa tggtcatatg

361 gccgtgcagc tgtggcacac cggacgcatt tctcacgcca gcctgcaacc tggcggtcag

421 gcaccggtag cgccttcagc acttagcgcg ggaacacgta cttctctgcg cgatgaaaat

481 ggtcaggcga tccgtgttga aacatccatg ccgcgtgcgc ttgaactgga agagattcca

541 ggtatcgtca atgatttccg tcaggccatt gctaacgcgc gtgaagccgg ttttgatctg 601 gtagagctcc actctgctca cggttatttg ctgcatcagt tcctttctcc ttcttcaaac

661 catcgtaccg atcagtacgg cggcagcgtg gaaaatcgcg cacgtttggt actggaagtg

721 gtcgatgccg ggattgaaga atggggtgcc gatcgcattg gcattcgcgt ttcaccaatc

781 ggtactttcc agaacacaga taacggcccg aatgaagaag ccgatgcact gtatctgatt

841 gaacaactgg gtaaacgcgg cattgcttat ctgcatatgt cagaaccaga ttgggcgggg

901 ggtgaaccgt atactgatgc gttccgcgaa aaagtacgcg cccgtttcca cggtccgatt 961 atcggcgcag gtgcatacac agtagaaaaa gctgaaacgc tgatcggcaa agggttaatt 1021 gatgcggtgg catttggtcg tgactggatt gcgaacccgg atctggtcgc ccgcttgcag 1081 cgcaaagctg agcttaaccc acagcgtgcc gaaagtttct acggtggcgg cgcggaaggc 1141 tataccgatt acccgacgtt gtaacgctga cgtcggtacc ctcgagtctg gtaaagaaac 1201 cgctgctgcg aaatttgaac gccagcacat ggactcgtct actagcgcag cttaattaac 1261 ctaggctgct gccaccgctg agcaataact agcataaccc cttggggcct ctaaacgggt 1321 cttgaggggt ttttgctga aacctcaggc atttgagaag cacacggtca cactgcttcc 1381 ggtagtcaat aaaccggtaa accagcaata gacataagcg gctatttaac gaccctgccc 
1441 tgaaccgacg accgggtcat cgtggccgga tcttgcggcc cctcggcttg aacgaattgt 1501 tagacattat ttgccgacta ccttggtgat ctcgcctttc acgtagtgga caaattcttc 1561 caactgatct gcgcgcgagg ccaagcgatc ttcttcttgt ccaagataag cctgtctagc 1621 ttcaagtatg acgggctgat actgggecgg caggcgctcc attgcccagt cggcagcgac 1681 atccttcggc gcgattttgc cggttactgc gctgtaccaa atgcgggaca acgtaagcac 1741 tacatttcgc tcatcgccag cccagtcggg cggcgagttc catagcgtta aggtttcatt 1801 tagcgcctca aatagatcct gttcaggaac cggatcaaag agttcctccg ccgctggacc 1861 taccaaggca acgctatgtt ctcttgcttt tgtcagcaag atagccagat caatgtcgat 1921 cgtggctggc tcgaagatac ctgcaagaat gtcattgcgc tgccattctc caaattgcag 1981 ttcgcgctta gctggataac gccacggaat gatgtcgtcg tgcacaacaa tggtgacttc 2041 tacagcgcgg agaatctcgc tctctccagg ggaagccgaa gtttccaaaa ggtcgttgat 2101 caaagctcgc cgcgttgttt catcaagcct tacggtcacc gtaaccagca aatcaatatc 2161 actgtgtggc ttcaggccgc catccactgc ggagccgtac aaatgtacgg ccagcaacgt 2221 cggttcgaga tggcgctcga tgacgccaac tacctctgat agttgagtcg atacttcggc 2281 gatcaccgct tccctcatac tcttcctttt tcaatattat tgaagcattt atcagggtta 2341 ttgtctcatg agcggataca tatttgaatg tatttagaaa aataaacaaa tagctagctc 2401 actcggtcge tacgctccgg gcgtgagact gcggcgggeg ctgcggacac atacaaagtt 2461 acccacagat tccgtggata agcaggggac taacatgtga ggcaaaacag cagggccgcg 2521 ccggtggcgt ttttccatag gctccgccct cctgccagag ttcacataaa cagacgcttt 2581 tccggtgcat ctgtgggagc cgtgaggctc aaccatgaat ctgacagtac gggcgaaacc 2641 cgacaggact taaagatccc caccgtttcc ggcgggtcgc tccctcttgc getctcctgt 2701 tccgaccctg ccgtttaccg gatacctgtt ccgcctttct cccttacggg aagtgtggcg 2761 ctttctcata gctcacacac tggtatctcg gctcggtgta ggtcgttcgc tccaagctgg 2821 gctgtaagca agaactcccc gttcagcccg actgctgcge cttatccggt aactgttcac 2881 ttgagtccaa cccggaaaag cacggtaaaa cgccactggc agcagccatt ggtaactggg 2941 agttcgcaga ggatttgttt agctaaacac gcggttgctc ttgaagtgtg cgccaaagtc 3001 cggctacact ggaaggacag atttggttgc tgtgctctgc gaaagccagt taccacggtt 3061 aagcagttcc ccaactgact taaccttcga tcaaaccacc tccccaggtg gtttttcgt 3121 ttacagggca aaagattacg cgcagaaaaa aaggatctca agaagatcct ttgatctttt 3181 ctactgaacc gctctagatt tcagtgcaat ttatctcttc aaatgtagca cctgaagtca 3241 gccccatacg atataagttg taattctcat gttagtcatg ccccgcgccc accggaagga 3301 gctgactggg ttgaaggctc tcaagggcat cggtcgag

$3188 \mathrm{bp}$ DNA circular DEFINITION nitrite sensing module vector FEATURES Location/Qualifiers

$$
\begin{array}{cc}
\text { CDS } & 1 . .678 \\
& \text { /product="RFP BBa_E1010" } \\
\text { /modified_by="User" } & \\
\text { /label="RFP BBa_E1010" } \\
\text { terminator } \quad 683 . .762 \\
\text { /note="BBa_B0010" } \\
\text { /modified_by="User" }
\end{array}
$$




$$
\begin{aligned}
& \text { /label="Terminator BBa_B0010" } \\
& \text { terminator } 771 . .811 \\
& \text { /note="BBa_B0012" } \\
& \text { /modified_by="User" } \\
& \text { /label="Terminator BBa_B0012" } \\
& \text { misc_feature complement(1029..1688) } \\
& \text { /product="chloramphenicol resistance marker" } \\
& \text { /modified_by="User" } \\
& \text { /label="CAT" } \\
& \text { rep_origin 2050..2962 } \\
& \text { /note="P15A" } \\
& \text { /modified_by="User" } \\
& \text { /label="P15A" } \\
& \text { promoter }<3043 . .>3188 \\
& \text { /note="pYeaR promoter region" } \\
& \text { /label="pYeaR promoter region" }
\end{aligned}
$$


1861 tctgcgaagt gatcttccgt cacaggtatt tattcggcgc aaagtgcgtc gggtgatgct 1921 gccaacttac tgatttagtg tatgatggtg tttttgaggt gctccagtgg cttctgtttc 1981 tatcagctgt ccctcctgtt cagctactga cggggtggtg cgtaacggca aaagcaccgc 2041 cggacatcag cgctagcgga gtgtatactg gcttactatg ttggcactga tgagggtgtc 2101 agtgaagtgc ttcatgtggc aggagaaaaa aggctgcacc ggtgcgtcag cagaatatgt 2161 gatacaggat atattccgct tcctcgctca ctgactcgct acgctcggtc gttcgactgc 2221 ggcgagcgga aatggcttac gaacggggcg gagatttcct ggaagatgcc aggaagatac 2281 ttaacaggga agtgagaggg ccgcggcaaa gccgttttc cataggctcc gcccccctga 2341 caagcatcac gaaatctgac gctcaaatca gtggtggcga aacccgacag gactataaag 2401 ataccaggcg tttcccctgg cggctccctc gtgcgctctc ctgttcctgc ctttcggttt 2461 accggtgtca ttccgctgtt atggccgcgt ttgtctcatt ccacgcctga cactcagttc 2521 cgggtaggca gttcgctcca agctggactg tatgcacgaa ccccccgttc agtccgaccg 2581 ctgcgcctta tccggtaact atcgtcttga gtccaacccg gaaagacatg caaaagcacc 2641 actggcagca gccactggta attgatttag aggagttagt cttgaagtca tgcgccggtt 2701 aaggctaaac tgaaaggaca agttttggtg actgcgctcc tccaagccag ttacctcggt 2761 tcaaagagtt ggtagctcag agaaccttcg aaaaaccgcc ctgcaaggcg gtttttcgt $2821 \mathrm{tttcagagca}$ agagattacg cgcagaccaa aacgatctca agaagatcat cttattaatc 2881 agataaaata tttctagatt tcagtgcaat ttatctcttc aaatgtagca cctgaagtca 2941 gccccatacg atataagttg taattctcat gttagtcatg ccccgcgccc accggaagga 3001 gctgactggg ttgaaggctc tcaagggcat cggtcgaggc ctttcccatc tataatcctc 3061 cctgattctt cgctgatatg gtgctaaaaa gtaaccaata aatggtattt aaaatgcaaa 3121 ttatcaggcg taccctgaaa cggctggaat aaaccgtttt cagcgcattc accgaaggag $/ /$ 3181 ggaaaagg

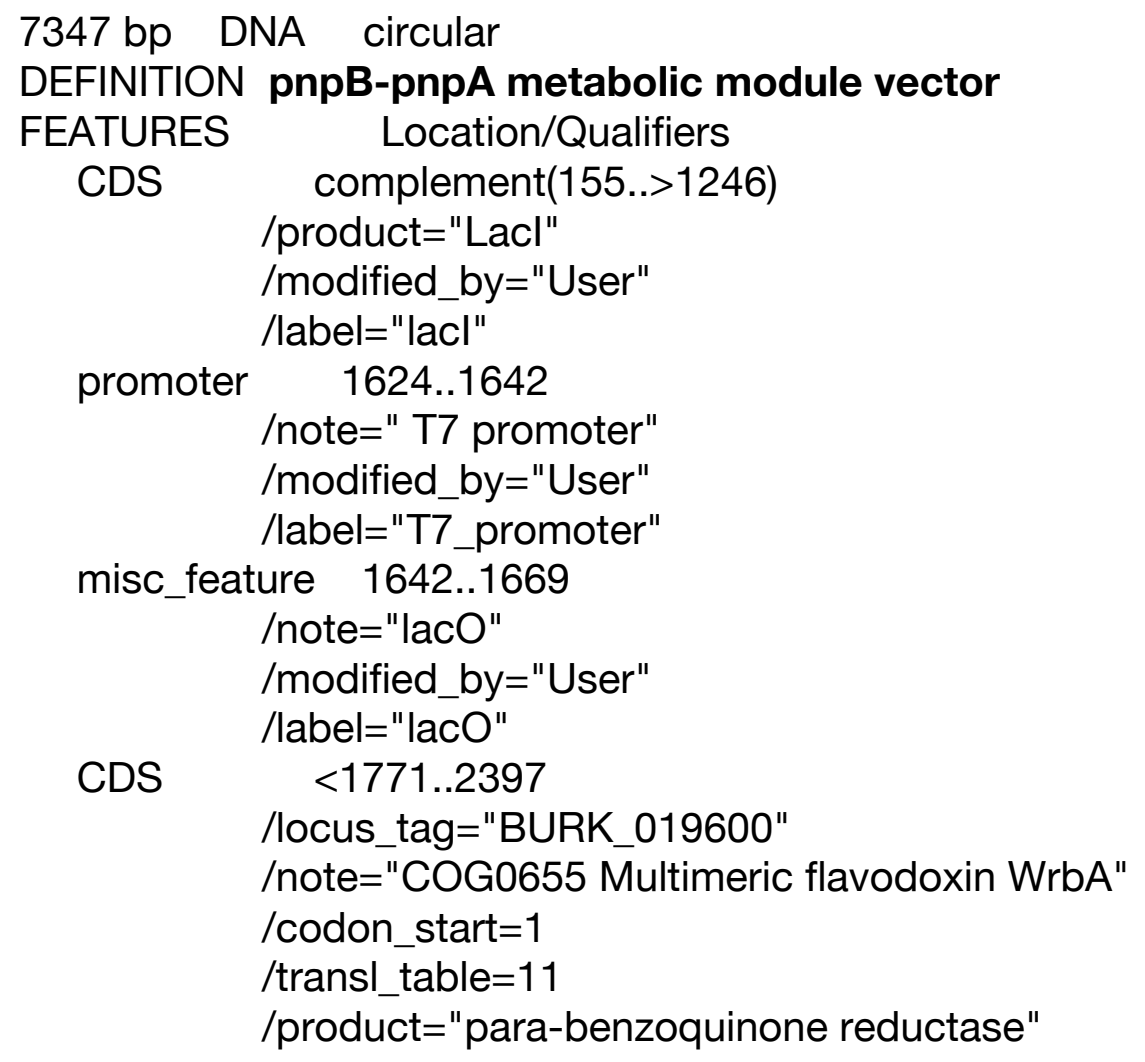


/protein_id="EKS70312.1"

/db_xref="Gl:413931025"

/translation="MATKIQIVFYSGYGHIYKMAEAIAAGVREVEDVEVKLLQVPELV

PEEVLVKSGMKGYRAAFESVQVATPEILAEADGIIFGTPTRFGNMCAQMRNFLDQTGG

LWMNGGLIGKVGSVFTSTASQHGGQETTISSFHTTLLHHGMVIVGVPYSEQRLVNMSE ISGGTPYGASTLAGADGSRQPSENELAIARFQGQHVATITKKLVAQSK" /modified_by="User"

/label="para-benzoquinone reductase CDS"

CDS 2425..3639

/locus_tag="BURK_019605"

/note="COG0654 2-polyprenyl-6-methoxyphenol hydroxylase

and related FAD-dependent oxidoreductases"

/codon_start=1

/transl_table $=11$

/product="para-nitrophenol 4-monooxygenase"

/protein_id="EKS70313.1"

/db_xref="Gl:413931026"

/translation="METLEGVVVVGGGPVGLLTALKLGRAGVKVIVLESEACVSPSPR

AVAYMPPTIAAMQRFGVLEDIRKRAVYCPSINYRHGDGTLFSTLEWSAVAEDTEFPYM

LLLGQNHVSNVIKQHLQTLPNVDIRWNHRVESIDQDANYVTIETRCPGGISRLRARWV

AATDGARSTVREKIGLSFDGITWAERLVATNVFYDFTVHGYSRANFVHDPLDWAVVVQ

LDQSGLWRVCYGEDPSISMEEVRRRMPERFKRLLPGAPTPDRYRVDHLNPYRVHQR $\mathrm{CA}$

AEFRRGRVILAGDAAHATNPMGGLGLSGGVLDAEHLARALISVVNDSAPVKVLDDYSV

DRRKVFLEFTSPTATQNFNWMKESDPAQRARDTELLTHAGTDRAVMREVLLSFEKLN $\mathrm{G}$

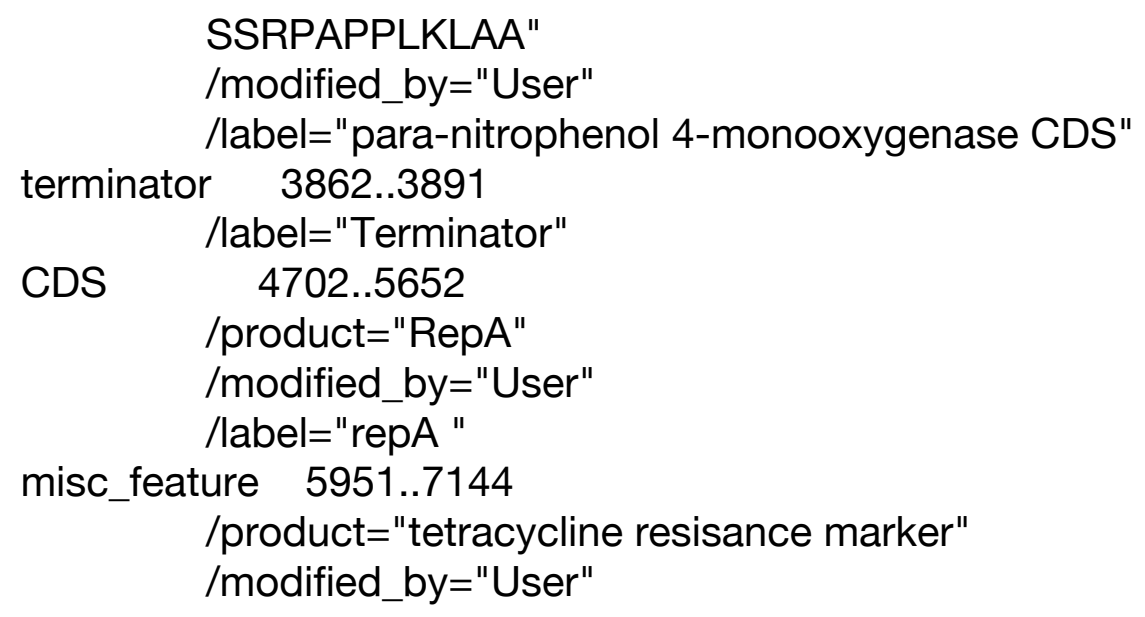




\section{ORIGIN}

$$
\text { /label="tetR CDS" }
$$

1 aattcgaaga cgtcagaaga cagtcataag tgcggcgacg atagtcatgc cccgcgccca

61 ccggaaggag ctgactgggt tgaaggctct caagggcatc ggtcgagatc ccggtgccta

121 atgagtgagc taacttacat taattgcgtt gcgctcactg cccgctttcc agtcgggaaa

181 cctgtcgtgc cagctgcatt aatgaatcgg ccaacgcgcg gggagaggcg gtttgcgtat

241 tgggcgccag ggtggttttt cttttcacca gtgagacggg caacagctga ttgcccttca

301 ccgcctggcc ctgagagagt tgcagcaagc ggtccacgct ggtttgcccc agcaggcgaa

361 aatcctgttt gatggtggtt aacggcggga tataacatga gctgtcttcg gtatcgtcgt

421 atcccactac cgagatatcc gcaccaacgc gcagcccgga ctcggtaatg gcgcgcattg

481 cgcccagcgc catctgatcg ttggcaacca gcatcgcagt gggaacgatg ccctcattca

541 gcatttgcat ggtttgttga aaaccggaca tggcactcca gtcgccttcc cgttccgcta

601 tcggctgaat ttgattgcga gtgagatatt tatgccagcc agccagacgc agacgcgccg

661 agacagaact taatgggccc gctaacagcg cgatttgctg gtgacccaat gcgaccagat

721 gctccacgcc cagtcgcgta ccgtcttcat gggagaaaat aatactgttg atgggtgtct

781 ggtcagagac atcaagaaat aacgccggaa cattagtgca ggcagcttcc acagcaatgg

841 catcctggtc atccagcgga tagttaatga tcagcccact gacgcgttgc gcgagaagat

901 tgtgcaccgc cgctttacag gettcgacgc cgcttcgttc taccatcgac accaccacgc

961 tggcacccag ttgatcggcg cgagatttaa tcgccgcgac aatttgcgac ggcgcgtgca

1021 gggccagact ggaggtggca acgccaatca gcaacgactg tttgcccgcc agttgttgtg

1081 ccacgcggtt gggaatgtaa ttcagctccg ccatcgccgc ttccactttt tcccgcgttt

1141 tcgcagaaac gtggctggcc tggttcacca cgcgggaaac ggtctgataa gagacaccgg

1201 catactctgc gacatcgtat aacgttactg gtttcacatt caccaccctg aattgactct

1261 cttccgggcg ctatcatgcc ataccgcgaa aggttttgcg ccattcgatg gtgtccggga

1321 tctcgacgct ctccettatg cgactcctgc attaggaagc agcccagtag taggttgagg

1381 ccgttgagca ccgccgccgc aaggaatggt gcatgcaagg agatggcgcc caacagtccc

1441 ccggccacgg ggcctgccac catacccacg ccgaaacaag cgctcatgag cccgaagtgg

1501 cgagcccgat cttccccatc ggtgatgtcg gcgatatagg cgccagcaac cgcacctgtg

1561 gcgccggtga tgccggccac gatgcgtccg gcgtagagga tcgagatctc gatcccgcga

1621 aattaatacg actcactata ggggaattgt gagcggataa caattcccct ctagaaataa

$1681 \mathrm{ttttgtttaa}$ ctttaagaag gagatatacc atgggcagca gccatcatca tcatcatcac

1741 agcagcggcc tggtgccgcg cggcagccat atggcaacta agattcagat tgtgtttac

1801 agcggctacg gccacatcta caagatggcc gaagctatcg cggcaggcgt cagagaagtt

1861 gaagacgttg aagtgaagtt gctccaggtt ccggaactcg tgcccgaaga agtcctggtt

1921 aagagcggaa tgaagggcta tcgcgctgca ttcgaaagtg ttcaggttgc tacgccggag

1981 attctcgcgg aagccgacgg catcatcttc ggtacgccga cgcgattcgg caacatgtgc

2041 gctcaaatgc gcaacttcct ggaccaaacg ggtggtctgt ggatgaacgg tggcctgatt

2101 ggcaaggtcg gtagcgtctt cacgagcacc gcttcgcaac acggtggtca agaaacgacc

2161 atcagcagct tccatacgac cttgcttcac cacggtatgg tcatcgttgg tgtgccgtat

2221 tcggagcagc gactggtcaa catgagcgag atttccggtg gcacgcccta cggtgcgtcg

2281 acgcttgcgg gtgcagatgg ttcgcgtcaa ccgagcgaaa acgaactggc aatcgctcgc

2341 ttccaaggtc agcatgttgc cacgatcacc aagaagctgg tcgcgcagtc caagtaactc

2401 ttaactttaa gaaggagata taccatggaa acgcttgaag gagtggtcgt tgttggtgga

2461 ggtcccgttg gcctgctcac cgcgctcaaa ctgggtcgcg ctggcgtcaa ggtcattgtg

2521 ctcgaatcgg aggcttgcgt ttcgccgtcg ccccgcgctg tcgcgtatat gcccccaacc

2581 atcgctgcga tgcagcggtt tggcgtgctt gaggatattc gcaagcgtgc agtgtattgc

2641 ccgagcatca actatcgtca cggcgacggc acgcttttca gtacgctgga atggagtgct

2701 gttgctgagg acaccgagtt cccgtacatg ttgctgctgg gccagaacca tgtatccaac 
2761 gtcattaagc agcaccttca aacgttgccc aacgtagata ttcgctggaa tcaccgcgtt 2821 gagagtatcg atcaagacgc caactacgtg acgattgaaa cgcgttgtcc gggtggcatt 2881 tcgcgactgc gcgctcgctg ggtggcggcg accgatgggg cacgcagcac ggtgcgcgag 2941 aagattggcc tgagctttga cggtatcact tgggcagagc gactcgtcgc aacgaatgtc 3001 ttctatgact tcaccgtcca tggctattcg cgtgccaatt tcgtgcatga cccgcttgac 3061 tgggcagtgg tggtgcagct tgaccaaagc ggettgtgge gcgtctgtta cggcgaagat 3121 ccgtcgatct cgatggagga agtgcgtcgt cgtatgcccg agcgtttcaa acgtctcttg 3181 ccgggagccc cgacacctga ccgttatcgc gtcgaccacc tgaatccgta ccgcgtacat 3241 cagcgctgtg ctgccgagtt tcgccgagge cgtgtgattc tcgcgggtga cgccgcgcac 3301 gcaacaaatc cgatgggtgg cctgggtctg tccggtggtg tgcttgatgc agaacatctg 3361 gctcgtgcgc tgatctcggt cgtcaacgac tccgcaccgg tcaaggtgct cgacgactac 3421 tcggttgatc gtcgaaaggt cttccttgag ttcacgtcgc cgacggcaac gcagaacttc 3481 aactggatga aggaaagcga ccctgcgcaa cgcgcccgtg ataccgaact gcttacacac 3541 gcaggcacgg accgcgctgt gatgcgcgag gttcttctga gcttcgagaa gctcaacggc 3601 agcagccgcc cggccccgcc tcttaagctt gcagcgtaaa gcgtcttcct gcaggagtca 3661 ctaagggtta gttagttaga ttagcagaaa gtcaaaagcc tccgaccgga ggcttttgac 3721 taaaacttcc cttggggtta tcattggggc tcactcaaag gcggtaatca gataaaaaaa 3781 atccttagct ttcgctaagg atgatttctg ctagagctgt cagaccaagt ttacgagctc 3841 gcttggactc ctgttgatag atccagtaat gacctcagaa ctccatctgg atttgttcag 3901 aacgctcggt tgccgccggg cgtttttat tggtgagaat ccaagcacta gggacagtaa 3961 gacgggtaag cctgttgatg ataccgctgc cttactgggt gcattagcca gtctgaatga 4021 cctgtcacgg gataatccga agtggtcaga ctggaaaatc agagggcagg aactgctgaa 4081 cagcaaaaag tcagatagca ccacatagca gacccgccat aaaacgccct gagaagcccg 4141 tgacgggctt ttcttgtatt atgggtagtt tccttgcatg aatccataaa aggcgcctgt 4201 agtgccattt acccccattc actgccagag ccgtgagcgc agcgaactga atgtcacgaa 4261 aaagacagcg actcaggtgc ctgatggtcg gagacaaaag gaatattcag cgatttgccc 4321 gagcttgcga gggtgctact taagccttta gggttttaag gtctgttttg tagaggagca 4381 aacagcgttt gcgacatcct tttgtaatac tgcggaactg actaaagtag tgagttatac 4441 acagggctgg gatctattct ttttatcttt ttttattctt tctttattct ataaattata 4501 accacttgaa tataaacaaa aaaaacacac aaaggtctag cggaatttac agagggtcta 4561 gcagaattta caagttttcc agcaaaggtc tagcagaatt tacagatacc cacaactcaa 4621 aggaaaagga catgtaatta tcattgacta gcccatctca attggtatag tgattaaaat 4681 cacctagacc aattgagatg tatgtctgaa ttagttgttt tcaaagcaaa tgaactagcg 4741 attagtcgct atgacttaac ggagcatgaa accaagctaa ttttatgctg tgtggcacta 4801 ctcaacccca cgattgaaaa ccctacaagg aaagaacgga cggtatcgtt cacttataac 4861 caatacgctc agatgatgaa catcagtagg gaaaatgctt atggtgtatt agctaaagca 4921 accagagagc tgatgacgag aactgtggaa atcaggaatc ctttggttaa aggctttgag 4981 attttccagt ggacaaacta tgccaagttc tcaagcgaaa aattagaatt agtttttagt 5041 gaagagatat tgccttatct tttccagtta aaaaaattca taaaatataa tctggaacat 5101 gttaagtctt ttgaaaacaa atactctatg aggatttatg agtggttatt aaaagaacta 5161 acacaaaaga aaactcacaa ggcaaatata gagattagcc ttgatgaatt taagttcatg 5221 ttaatgcttg aaaataacta ccatgagttt aaaaggctta accaatgggt tttgaaacca 5281 ataagtaaag atttaaacac ttacagcaat atgaaattgg tggttgataa gcgaggccgc 5341 ccgactgata cgttgatttt ccaagttgaa ctagatagac aaatggatct cgtaaccgaa $5401 \mathrm{cttgagaaca}$ accagataaa aatgaatggt gacaaaatac caacaaccat tacatcagat 5461 tcctacctac gtaacggact aagaaaaaca ctacacgatg ctttaactgc aaaaattcag 5521 ctcaccagtt ttgaggcaaa attttgagt gacatgcaaa gtaagcatga tctcaatggt 5581 tcgttctcat ggctcacgca aaaacaacga accacactag agaacatact ggctaaatac 
5641 ggaaggatct gaggttctta tggctcttgt atctatcagt gaagcatcaa gactaacaaa 5701 caaaagtaga acaactgttc accgttagat atcaaaggga aaactgtcca tatgcacaga 5761 tgaaaacggt gtaaaaaaga tagatacatc agagctttta cgagtttttg gtgcatttaa 5821 agctgttcac catgaacaga tcgacaatgt aactactaga ggagattctc atgtttgaca 5881 gcttatcatc gataagcttt aatgcggtag tttatcacag ttaaattgct aacgcagtca 5941 ggcaccgtgt atgaaatcta acaatgcgct catcgtcatt ctcggcaccg tcaccctgga 6001 cgctgtaggc ataggcttgg ttatgccggt actgccgggc ctcttgcggg atatcgtcca 6061 ttccgacagt attgccagtc actatggcgt gctgcttgcg ctctatgcgt tgatgcaatt 6121 tctttgcgca cccgttctcg gagccctgtc cgaccgcttt ggccgccgtc cagtcctgct 6181 cgcttcgctc cttggagcca ctatcgacta cgcgatcatg gcgaccacac ccgtcctgtg 6241 gattctctac gccggacgca tcgtggcggg catcacgggt gccacaggtg cggttgctgg 6301 tgcctatatc gccgacatca ccgatgggga agatcgggct cgccacttcg ggctcatgag 6361 cgcttgtttc ggcgtgggta tggtggcagg ccccgtggcc gggggactgt tgggtgccat 6421 ctccttgcat gcaccattcc ttgcggcgge ggtgctcaac ggcctcaacc tcctcctggg 6481 ctgcttcctt atgcaggaat cgcataaggg agagcgccgt ccgatgccct tgcgtgcctt 6541 caatccagtc agctccttcc ggtgggcgcg gggcatgact atcgtcgccg cacttatgac 6601 tgtttcttt atcatgcaac tcgtaggaca ggttccggca gcgctctggg tcatttcgg 6661 cgaggaccgc tttcgctgga gcgcgacgat gatcggcctg tcgcttgcgg tattcggaat 6721 cttgcacgcc ctcgctcaag cettcgtcac gggccccgcc accaaacgtt tcggcgagaa 6781 gcaggccatt atcgcgggca tggcggccga cgcgctgggc tacgtcttgc tggcgttcgc 6841 gacgcgcggc tggatggcct tccccattat gattcttctc gettccggcg gcatcggtat 6901 gcccgcgttg caggccatgc tgtcccgcca agtagatgac gaccatcagg gacagcttca 6961 agggtcgctc gcggctctta ccagcctcac ttcgatcatt ggaccgctga tcgtcacggc 7021 gatttatgcc gcctcggcga gcacatggaa cgggttggca tggattgtag gtgccgccct 7081 ttaccttgtc tgcctccccg cgttgcgtcg cggtgcatgg agccgggeca cctcgaccta 7141 ataatactag ctccggcaaa aaaacgggca aggtgtcacc accctgccct ttttctttaa 7201 aaccgaaaag attacttcgc gtttgccacc tgacgtctaa gaaaaggaat attcagcaat 7261 ttgcccgtgc cgaagaaagg cccacccgtg aaggtgagcc agtgagttga ttgctacgta // 7321 attagttagt tagcccttag tgactcg

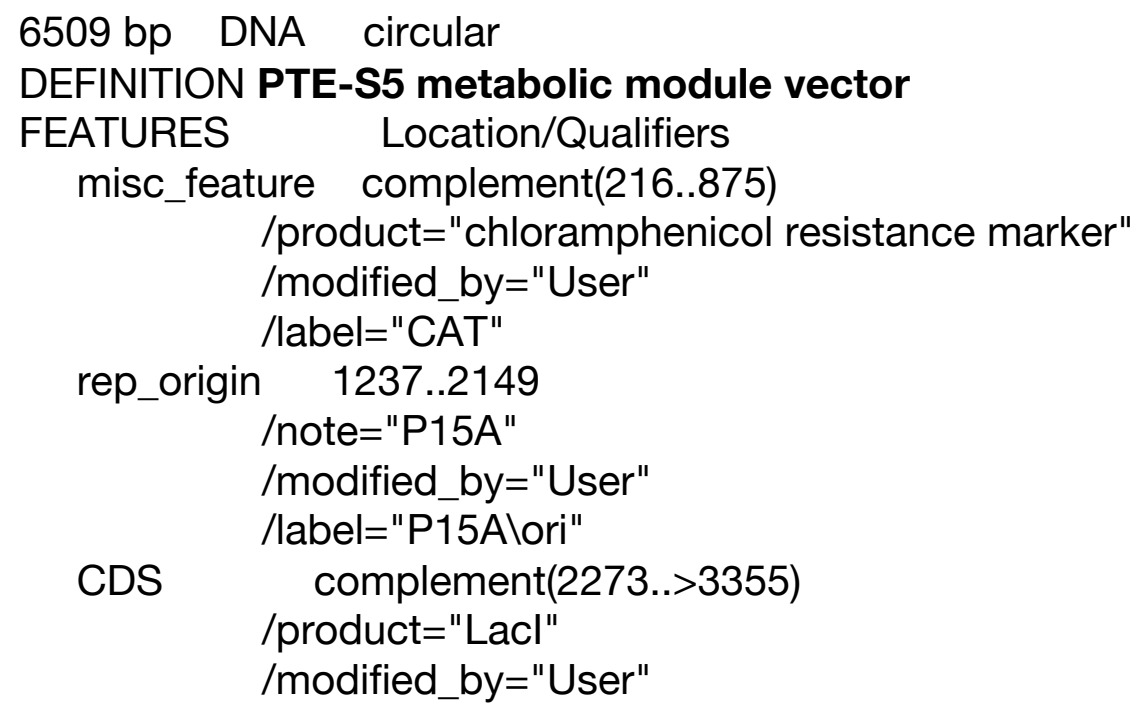




$\begin{array}{cl}\text { /label="lacl" } \\ \text { promoter } & \text { 3545..3617 } \\ & \text { /note="TRC promoter" } \\ & \text { /modified_by="User" } \\ \text { /label="trc prom" } & \\ \text { misc_feature } 3576 . .3603 \\ \\ \text { /note="LacO" } \\ \text { /modified_by="User" } \\ \text { /label="lacO reg" } \\ \text { CDS } \quad 3667 . .5850 \\ \\ \text { /product="MBP-PTE-S5" } \\ \text { /label="MBP-PTE-S5" }\end{array}$

1 aacctcaggc atttgagaag cacacggtca cactgcttcc ggtagtcaat aaaccggtaa 61 accagcaata gacataagcg gctatttaac gaccctgccc tgaaccgacg accgggtcga 121 atttgctttc gaatttctgc cattcatccg cttattatca cttattcagg cgtagcacca 181 ggcgtttaag ggcaccaata actgccttaa aaaaattacg ccccgccctg ccactcatcg 241 cagtactgtt gtaattcatt aagcattctg ccgacatgga agccatcaca gacggcatga 301 tgaacctgaa tcgccagcgg catcagcacc ttgtcgcctt gcgtataata tttgcccata 361 gtgaaaacgg gggcgaagaa gttgtccata ttggccacgt ttaaatcaaa actggtgaaa 421 ctcacccagg gattggctga gacgaaaaac atattctcaa taaacccttt agggaaatag 481 gccaggtttt caccgtaaca cgccacatct tgcgaatata tgtgtagaaa ctgccggaaa 541 tcgtcgtggt attcactcca gagcgatgaa aacgtttcag tttgctcatg gaaaacggtg 601 taacaagggt gaacactatc ccatatcacc agctcaccgt ctttcattgc catacggaac 661 tccggatgag cattcatcag gcgggcaaga atgtgaataa aggccggata aaacttgtgc 721 ttatttttct ttacggtctt taaaaaggcc gtaatatcca gctgaacggt ctggttatag 781 gtacattgag caactgactg aaatgcctca aaatgttctt tacgatgcca ttgggatata 841 tcaacggtgg tatatccagt gattttttc tccattttag cttccttagc tcctgaaaat 901 ctcgataact caaaaaatac gcccggtagt gatcttattt cattatggtg aaagttggaa 961 cctcttacgt gccgatcaac gtctcatttt cgccaaaagt tggcccaggg cttcccggta 1021 tcaacaggga caccaggatt tatttattct gcgaagtgat cttccgtcac aggtatttat 1081 tcggcgcaaa gtgcgtcggg tgatgctgcc aacttactga tttagtgtat gatggtgttt 1141 ttgaggtgct ccagtggctt ctgtttctat cagctgtccc tcctgttcag ctactgacgg 1201 ggtggtgcgt aacggcaaaa gcaccgccgg acatcagcgc tagcggagtg tatactggct 1261 tactatgttg gcactgatga gggtgtcagt gaagtgcttc atgtggcagg agaaaaaagg 1321 ctgcaccggt gcgtcagcag aatatgtgat acaggatata ttccgcttcc tcgctcactg 1381 actcgctacg ctcggtcgtt cgactgcggc gagcggaaat ggcttacgaa cggggcggag 1441 atttcctgga agatgccagg aagatactta acagggaagt gagagggccg cggcaaagcc 1501 gtttttccat aggctccgcc cccctgacaa gcatcacgaa atctgacgct caaatcagtg 1561 gtggcgaaac ccgacaggac tataaagata ccaggcgttt cccctggcgg ctccctcgtg 1621 cgctctcctg ttcctgcctt tcggtttacc ggtgtcattc cgctgttatg gccgcgtttg 1681 tctcattcca cgcctgacac tcagttccgg gtaggcagtt cgctccaagc tggactgtat 1741 gcacgaaccc cccgttcagt ccgaccgctg cgccttatcc ggtaactatc gtcttgagtc 1801 caacccggaa agacatgcaa aagcaccact ggcagcagcc actggtaatt gatttagagg 1861 agttagtctt gaagtcatgc gccggttaag gctaaactga aaggacaagt tttggtgact 1921 gcgctcctcc aagccagtta cctcggttca aagagttggt agctcagaga accttcgaaa 1981 aaccgccctg caaggcggtt ttttcgtttt cagagcaaga gattacgcgc agaccaaaac 2041 gatctcaaga agatcatctt attaatcaga taaaatattt ctagatttca gtgcaattta 
2101 tctcttcaaa tgtagcacct gaagtcagcc ccatacgata taagttgtaa ttctcatgtt 2161 agtcatgccc cgcgcccacc ggaaggagct gactgggttg aaggctctca agggcatcgg 2221 tcgagatccc ggtgcctaat gagtgagcta acttacatta attgcgttgc gctcactgcc 2281 cgcttccag tcgggaaacc tgtcgtgcca gctgcattaa tgaatcggcc aacgcgcggg 2341 gagaggcggt ttgcgtattg ggcgccaggg tggttttct tttcaccagt gagacgggca 2401 acagctgatt gccettcacc gcctggccct gagagagttg cagcaagcgg tccacgctgg 2461 tttgccccag caggcgaaaa tcctgtttga tggtggttaa cggcgggata taacatgagc 2521 tgtcttcggt atcgtcgtat cccactaccg agatgtccgc accaacgcgc agcccggact 2581 cggtaatggc gcgcattgcg cccagcgcca tctgatcgtt ggcaaccagc atcgcagtgg 2641 gaacgatgcc ctcattcagc atttgcatgg tttgttgaaa accggacatg gcactccagt 2701 cgccttcccg ttccgctatc ggctgaattt gattgcgagt gagatattta tgccagccag 2761 ccagacgcag acgcgccgag acagaactta atgggcccgc taacagcgcg atttgctggt 2821 gacccaatgc gaccagatgc tccacgccca gtcgcgtacc gtcttcatgg gagaaaataa 2881 tactgttgat gggtgtctgg tcagagacat caagaaataa cgccggaaca ttagtgcagg 2941 cagcttccac agcaatggca tcctggtcat ccagcggata gttaatgatc agcccactga 3001 cgcgttgcgc gagaagattg tgcaccgccg ctttacaggc ttcgacgccg cttcgttcta 3061 ccatcgacac caccacgctg gcacccagtt gatcggcgcg agatttaatc gccgcgacaa $3121 \mathrm{tttgcgacgg}$ cgcgtgcagg gccagactgg aggtggcaac gccaatcagc aacgactgtt 3181 tgcccgccag ttgttgtgcc acgcggttgg gaatgtaatt cagctccgcc atcgccgctt 3241 ccactttttc ccgcgtttc gcagaaacgt ggctggcctg gttcaccacg cgggaaacgg 3301 tctgataaga gacaccggca tactctgcga catcgtataa cgttactggt ttcacattca 3361 ccaccctgaa ttgactctct tccgggcgct atcatgccat accgcgaaag gttttgcgcc 3421 attcgatggt gtccgggatc tcgacgctct cccttatgcg actcctgcag gcgcactccc 3481 gttctggata atgtttttg cgccgacatc ataacggttc tggcaaatat tctgaaatga 3541 gctgttgaca attaatcatc ggctcgtata atgtgtggaa ttgtgagcgg ataacaattt 3601 cacacaggaa acagccagtc cgtttaggtg ttttcacgag cacttcacca acaaggacca 3661 tagcatatga aaatcgaaga aggtaaactg gtaatctgga ttaacggcga taaaggctat 3721 aacggtctcg ctgaagtcgg taagaaattc gagaaagata ccggaattaa agtcaccgtt 3781 gagcatccgg ataaactgga agagaaattc ccacaggttg cggcaactgg cgatggccct 3841 gacattatct tctgggcaca cgaccgcttt ggtggctacg ctcaatctgg cctgttggct 3901 gaaatcaccc cggacaaagc gttccaggac aagctgtatc cgtttacctg ggatgccgta 3961 cgttacaacg gcaagctgat tgcttacccg atcgctgttg aagcgttatc gctgatttat 4021 aacaaagatc tgctgccgaa cccgccaaaa acctgggaag agatcccggc gctggataaa 4081 gaactgaaag cgaaaggtaa gagcgcgctg atgttcaacc tgcaagaacc gtacttcacc 4141 tggccgctga ttgctgctga cgggggttat gcgttcaagt atgaaaacgg caagtacgac 4201 attaaagacg tgggcgtgga taacgctggc gcgaaagcgg gtctgacctt cctggttgac 4261 ctgattaaaa acaaacacat gaatgcagac accgattact ccatcgcaga agctgccttt 4321 aataaaggcg aaacagcgat gaccatcaac ggcccgtggg catggtccaa catcgacacc 4381 agcaaagtga attatggtgt aacggtactg ccgaccttca agggtcaacc atccaaaccg 4441 ttcgttggcg tgctgagcgc aggtattaac gccgccagtc cgaacaaaga gctggcaaaa 4501 gagttcctcg aaaactatct gctgactgat gaaggtctgg aagcggttaa taaagacaaa 4561 ccgctgggtg ccgtagcgct gaagtcttac gaggaagagt tggcgaaaga tccacgtatt 4621 gccgccacta tggaaaacgc ccagaaaggt gaaatcatgc cgaacatccc gcagatgtcc 4681 gctttctggt atgccgtgcg tactgcggtg atcaacgccg ccagcggtcg tcagactgtc 4741 gatgaagcc tgaaagacgc gcagactaat tcgagctcga acaacaacaa caataacaat 4801 aacaacaacc tcgggatcga gggaaggatt tcagaattca tcaccaacag cggcgatcgg 4861 atcaataccg tgcgcggtcc tatcacaatc tctgaagcgg gtttcacact gactcacgag 4921 cacatctgcg gcagctcggc aggattcttg cgtgcttggc cggagttctt cggtagccgc 
4981 aaagccctag cggaaaaggc tgtgagagga ttgcgccgcg ccagagcggc tggcgtgcga 5041 acgattgtcg atgtgtcgac tttcgatatc ggtcgcgatg tcagtttatt ggccgaggtt 5101 tcgcgggctg ccgacgttca tatcgtggcg gcgaccggct tgtggttcga cccgccactt 5161 tcgatgcgat tgaggagtgt agaggaactc acacagttct tcctgcgtga gattcaatat 5221 ggcatcgaag acaccggaat tagggcgggc attatcaagg tcgcgaccac aggcaaggcg 5281 accccctttc aggagttagt gttaagggcg gccgcccggg ccagcttggc caccggtgtt 5341 ccggtaacca ctcacacggc agcaagtcag cgcggtggtg agcagcaggc cgccattttt 5401 gagtccgaag gcttgagccc ctcacgggtt tgtattggtc acagcgatga tactgacgat 5461 ttgagctatc tcaccgccct cgctgcgcgc ggatacctca tcggtctaga ccacatcccg 5521 cacagtgcga ttggtctaga agataatgcg agtgcatcag ccctcctggg catccgttcg 5581 tggcaaacac gggctctctt gatcaaggcg ctcatcgacc aaggctacat gaaacaaatc 5641 ctcgtttcga atgactggct gttcgggttt tcgagctatg tcaccaacat catggacgtg 5701 atggatagcg tgaaccccga cgggatggcc ttcattccac tgagagtgat cccattccta 5761 cgagagaagg gcgtcccaca ggaaacgctg gcaggcatca ctgtgactaa cccggcgcgg 5821 ttcttgtcac cgaccttgcg ggcgtcatga ctgcaggcaa gettggcact ggccgtcgtt 5881 ttacaacgtc gtgactggga aaaccctggc gttacccaac ttaatcgcct tgcagcacat 5941 ccccctttcg ccagctggcg taatagcgaa gaggcccgca ccgatcgccc ttcccaacag 6001 ttgcgcagcc tgaatggcga atggcagctt ggctgttttg gcggatgaga taagattttc 6061 agcctgatac agattaaatc agaacgcaga agcggtctga taaaacagaa tttgcctggc 6121 ggcagtagcg cggtggtccc acctgacccc atgccgaact cagaagtgaa acgccgtagc 6181 gccgatggta gtgtggggtc tccccatgcg agagtaggga actgccaggc atcaaataaa 6241 acgaaaggct cagtcgaaag actgggcctt tcgttttatc tgttgtttgt cggtgaacgc 6301 tctcctgagt aggacaaatc cgccgggagc ggatttgaac gttgcgaagc aacggcccgg 6361 agggtggcgg gcaggacgcc cgccataaac tgccaggcat caaattaagc agaaggccat 6421 cctgacggat ggcctttttg cgtttctaca aactcttttt gtttattttt ctaaatacat 6481 tcaaatatgt atccgctcat gagacaata 